\title{
Depression, Treatment Response to TNFi, and Economic Outcomes among Working-Age Adults with Rheumatoid Arthritis
}

Arijita Deb

Follow this and additional works at: https://researchrepository.wvu.edu/etd

\section{Recommended Citation}

Deb, Arijita, "Depression, Treatment Response to TNFi, and Economic Outcomes among Working-Age Adults with Rheumatoid Arthritis" (2017). Graduate Theses, Dissertations, and Problem Reports. 5457. https://researchrepository.wvu.edu/etd/5457

This Dissertation is protected by copyright and/or related rights. It has been brought to you by the The Research Repository @ WVU with permission from the rights-holder(s). You are free to use this Dissertation in any way that is permitted by the copyright and related rights legislation that applies to your use. For other uses you must obtain permission from the rights-holder(s) directly, unless additional rights are indicated by a Creative Commons license in the record and/ or on the work itself. This Dissertation has been accepted for inclusion in WVU Graduate Theses, Dissertations, and Problem Reports collection by an authorized administrator of The Research Repository @ WVU.

For more information, please contact researchrepository@mail.wvu.edu. 
Depression, Treatment Response to TNFi, and Economic Outcomes among Working-Age

Adults with Rheumatoid Arthritis

\author{
Arijita Deb \\ Dissertation submitted to the School of Pharmacy \\ at West Virginia University \\ in partial fulfillment of the requirements \\ for the degree of \\ Doctor of Philosophy \\ in \\ Health Services and Outcomes Research \\ Usha Sambamoorthi, PhD, Chair \\ Nilanjana Dwibedi, PhD \\ Traci LeMasters, PhD \\ JoAnn Hornsby, MD \\ Wenhui Wei, PhD \\ Department of Pharmaceutical Systems and Policy
}

Morgantown, West Virginia

2017

Keywords: rheumatoid arthritis, depression, burden, tumor necrosis factor inhibitors, effectiveness

Copyright 2017 Arijita Deb 


\section{ABSTRACT \\ Depression, Treatment Response to TNFi, and Economic Outcomes among Working-Age Adults with Rheumatoid Arthritis}

\section{Arijita Deb}

Depression is one of the most frequently occurring conditions in Rheumatoid Arthritis (RA) patients and understanding depression as a comorbidity in RA is very important. The link between RA and depression is very complex and influenced by a multitude of factors such as shared pathophysiologic pathways of inflammation, RA-related treatment response, as well as the patient's demographic and socio-economic factors, and general difficulties in coping with the disease. Depression can increase the clinical, humanistic, and economic burden among RA patients. Growing evidence now suggests that depression is a systemic inflammatory condition and may exert a negative influence on the treatment response in RA by its effect on proinflammatory cytokines. Therefore, it is important to understand how depression is related to the treatment effectiveness of specific cytokine inhibitor drugs such as Tumor Necrosis Factor Inhibitor (TNFi) therapy that are used for the treatment of RA. The powerful anti-inflammatory effect of TNFi may have a potential beneficial effect in reducing the risk of depression in RA patients. It is important to gain a comprehensive understanding of how depression affects RA and how RA can affect depression in the real-world clinical practice settings, particularly among working-age individuals as the burden of depression is highest among working-age population. Therefore, this dissertation had three related aims focusing on working-age adults: 1) estimating the clinical, humanistic and economic burden of depression in RA; 2) examining the relationship between prevalent depression and treatment response to TNFi therapy in RA; and 3) evaluating the association between TNFi therapy and the risk of developing depression in RA. The study used data from multiple nationally representative sources to triangulate the complex relationship 
between RA and depression in working-age adults. These data sources were: the nationally representative Medical Expenditure Panel Survey (MEPS) for years 2009, 2011, 2013, and 2015 and a retrospective claims database for commercially insured working-age adults for the years 2009 through 2015. We found that one in every four working-age RA patients reported the presence of depression. RA patients with depression compared to RA patients without depression were significantly more likely to have pain-related interference with normal work, functional limitations, lower health-related quality of life (HRQoL) scores in the mental domain, higher annual healthcare expenditures and out-of-pocket spending burden, higher rate of unemployment, higher number of missed work days annually and higher lost annual wages due to missed work days. Depression was independently associated with treatment response to TNFi, even after adjusting for baseline predisposing, enabling, need and external environment factors among working-age RA patients. Among RA patients with depression, nearly three in every ten patients responded to TNFi therapy, whereas among RA patients without depression, nearly four in every ten patients responded to TNFi therapy. In addition, in a depression-free cohort of RA patients, those who responded to TNFi therapy were significantly less likely to develop newlydiagnosed depression as compared to those who did not respond to TNFi therapy. In summary, these findings underscore the importance of effectively managing depression in routine clinical practice of RA patients to reduce pain, functional or activity limitations, improve quality of life, lower direct and indirect healthcare costs. In addition, the findings highlight the negative impact of depression on treatment response to TNFi and provide insights into specific subgroups of RA patients with depression who are at risk of responding poorly to TNFi. Furthermore, reduction in the risk of depression in TNFi responders suggests that TNFi therapy is important not only for clinical improvement in RA disease but also for reducing the risk of depression. 


\section{Acknowledgement}

First and foremost, I would like to take this opportunity to thank Dr. Usha Sambamoorthi, my advisor and dissertation chair, whose relentless guidance, support and encouragement to pursue my research interests were instrumental for the completion of this dissertation. It was a great privilege to have her as my advisor and I have been immensely benefitted by taking the courses that she has offered and by working with her on several projects. Her constant oversight, thoughtful advice, and prompt feedback were absolutely invaluable. I would also like to express my sincere gratitude to my committee members Dr. Nilanjana Dwibedi, Dr. Wenhui Wei, Dr. Traci LeMasters and Dr. JoAnn Hornsby for their scientific contributions, ideas, and valuable suggestions. Special thanks to Dr. Nilanjana Dwibedi, Dr. Suresh Madhavan and Dr. Xi Tan in helping me with the data acquisition. I would also like to sincerely acknowledge and thank all the faculty and graduate students of the PSP department for their guidance and support. Last but not the least, I am extremely thankful to my parents and Sumit for their unconditional love, support, and insipration. 


\section{Table of Contents}

List of Tables ..................................................................................................................... vii

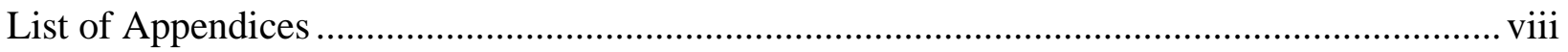

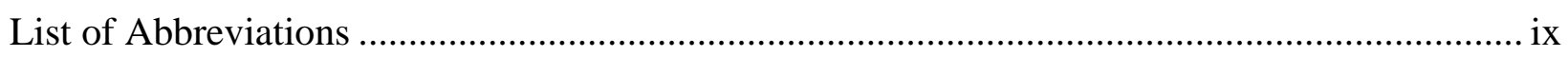

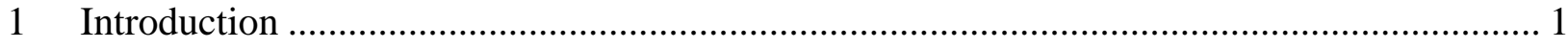

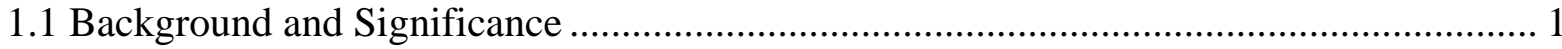

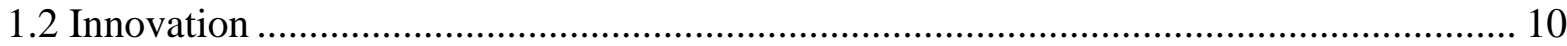

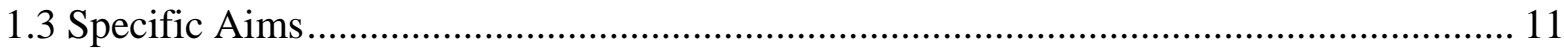

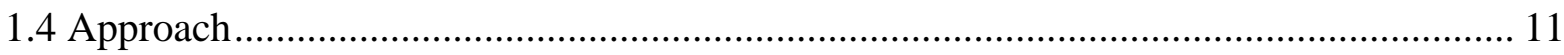

2 Burden of Depression among Working-Age Adults with Rheumatoid Arthritis .................. 14

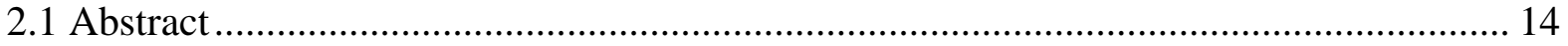

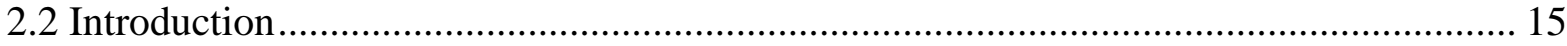

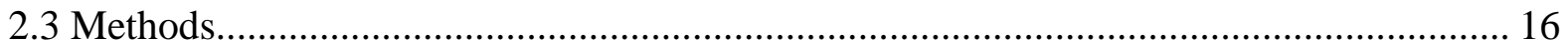

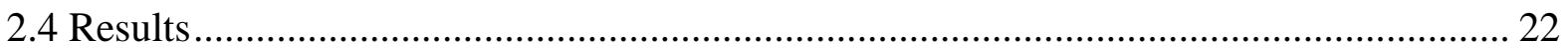

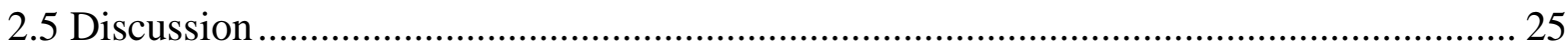

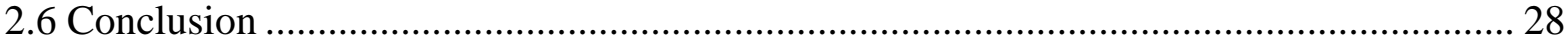

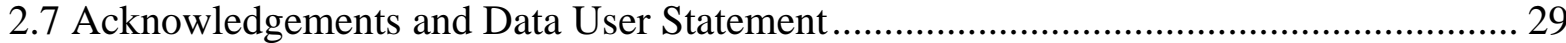

3 Depression and Treatment Response to Tumor Necrosis Factor Inhibitor Therapy among Working-Age Adults with Rheumatoid Arthritis: A Post Regression Non-Linear Decomposition

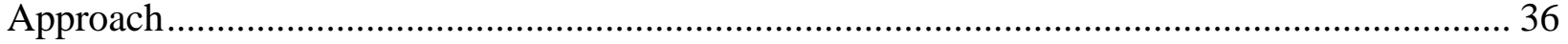

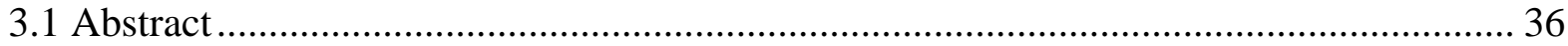

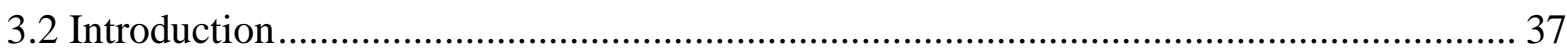

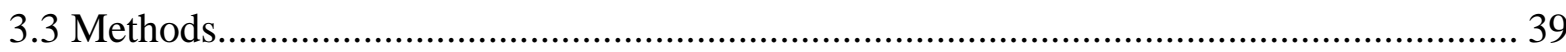

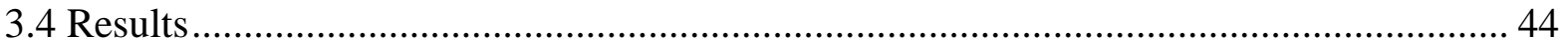

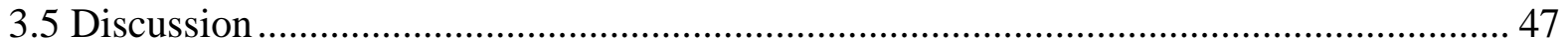

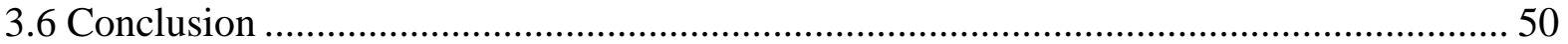

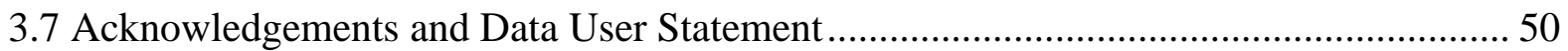

4 Tumor Necrosis Factor Inhibitor Therapy and the Risk of Developing Depression among Working Age Adults with Rheumatoid Arthritis ......................................................................... 57

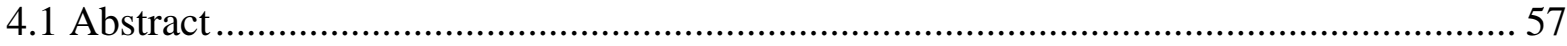

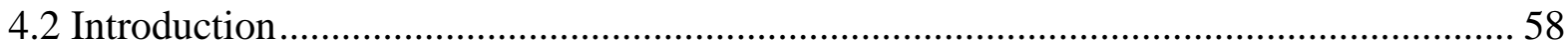

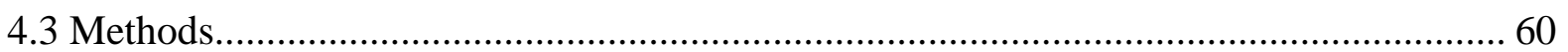

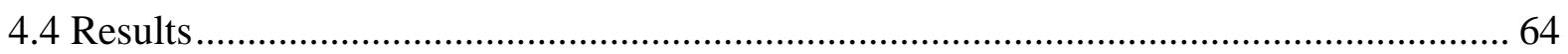




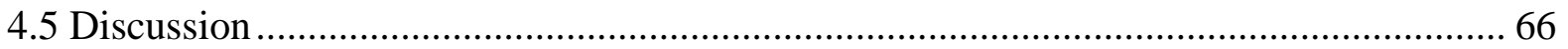

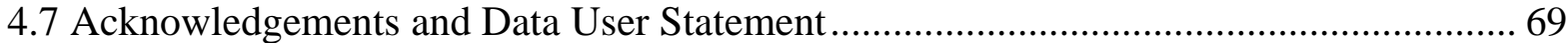

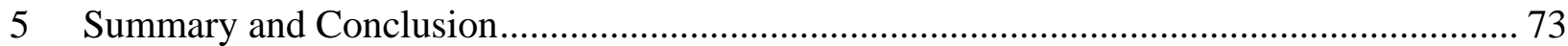

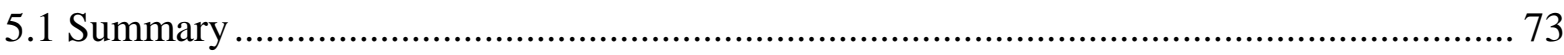

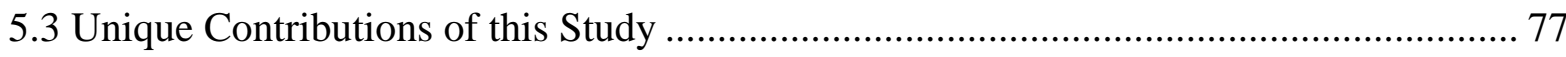

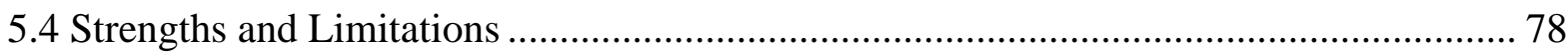

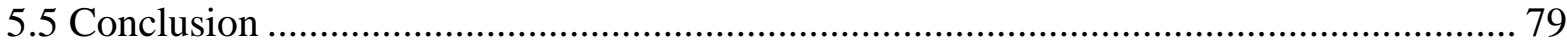

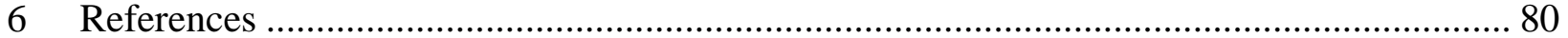

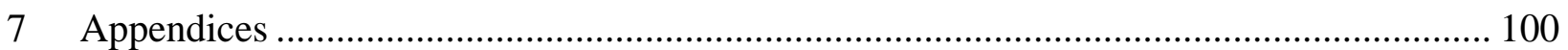




\section{List of Tables}

Table 2.1. Description of Study Sample by Depression among Working-Age (18 - 64 years) Adults with Rheumatoid Arthritis, using Medical Expenditure Panel Survey (2009, 2011, 2013

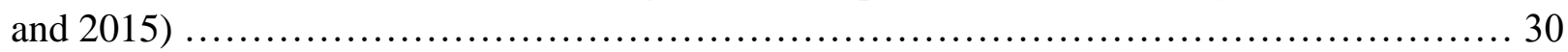

Table 2.2. Clinical Outcomes Associated with Depression among Working-Age Adults with Rheumatoid Arthritis, Medical Expenditure Panel Survey (2009, 2011, 2013 and 2015) ....... 32

Table 2.3. Humanistic Outcomes (Health-Related Quality of Measures) by Presence of Depression among Working Age Adults with Rheumatoid Arthritis, using Medical Expenditure Panel Survey $(2009,2011,2013$ and 2015) ........................................ 33

Table 2.4. Economic Outcomes by Presence of Depression among Working Age Adults with Rheumatoid Arthritis, Medical Expenditure Panel Survey (2009, 2011, 2013 and 2015) ....... 34

Table 3.1. Description of Working-Age Adults with Rheumatoid Arthritis initiated on TNFi by Depression Status During Baseline, using QuintilesIMS Real-World Data Adjudicated Claims Database, 2009-2015

Table 3.2. Components of the Algorithm for TNFi Response Status at 1 year after TNFi Initiation Working-Age Adults with Rheumatoid Arthritis initiated on TNFi by Depression Status, using QuintilesIMS Real-World Data Adjudicated Claims Database, 2009-2015 ........ 53

Table 3.3. Adjusted Odds Ratios (AOR) and 95\% Confidence intervals (CI) from Separate Logistic Regressions on Response to TNFi by Depression Status Working-Age Adults with Rheumatoid Arthritis Initiated on TNFi, using QuintilesIMS Real-World Data Adjudicated Claims Database, 2009-2015

Table 3.4. Contribution of Individual Characteristics to the Observed Difference in TNFi Treatment Response Rate by Depression Status Working-Age Adults with Rheumatoid Arthritis Initiated on TNFi, using QuintilesIMS Real-World Data Adjudicated Claims Database, 2009 2015

Table 4.1. Components of the Algorithm for TNFi Response Status at 1 year after TNFi Initiation

Table 4.2. Selected Baseline Characteristics of TNFi Responders vs. TNFi Non-Responders.. 71

Table 4.3. Adjusted Odds Ratio and 95\% Confidence Intervals of Selected Significant Variables from Logistic Regression on Newly Diagnosed Depression among Working-Age Adults with RA initiated on TNFi, using QuintilesIMS Real-World Data Adjudicated Claims Database 


\section{List of Appendices}

Appendix 7.1. Chapter 2. Description of Study Sample Working-Age (18 to 64 years) Adults with Rheumatoid Arthritis, using Medical Expenditure Panel Survey (2009, 2011, 2013 and

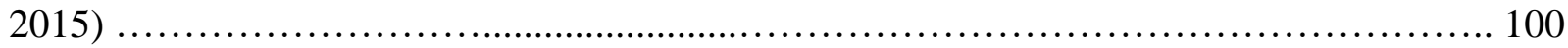

Appendix 7.2. Chapter 4. Baseline Characteristics of Working-Age (18 to 62 years) RA Patients Initiated on TNFi therapy from QuintilesIMS RWD Adjudicated Claims Database 102

Supplemental Figure 7.1.1. Chapter 3. Flow Diagram of Study Cohort .......................... 104

Supplemental Figure 7.1.2. Chapter 4. Flow Diagram of Study Cohort ............................ 105 


\section{List of Abbreviations}

\begin{tabular}{|c|c|}
\hline AHRQ & Agency for Healthcare Research and Quality \\
\hline ADL & Activities of Daily Living \\
\hline AOR & Adjusted Odds Ratio \\
\hline AUC & Area Under the Curve \\
\hline CI & Confidence Interval \\
\hline CRP & C-Reactive Protein \\
\hline $\mathrm{COL}$ & Column \\
\hline DAS-28 & Disease Activity Soring in 28 joints \\
\hline ED & Emergency Department \\
\hline FDA & Food and Drug Administration \\
\hline GLM & Generalized Linear Model \\
\hline HMO & Health Maintenance Organization \\
\hline HRQOL & Health Related Quality of Life \\
\hline OOP & Out-of-Pocket \\
\hline OR & Odds Ratio \\
\hline IADL & Instrumental activities of daily living \\
\hline ICD-9-CM & International Classification of Diseases, Ninth Revision, Clinical Modification \\
\hline IL-1 & Interleukin-1 \\
\hline IL-6 & Interleukin-6 \\
\hline IPTW & Inverse Probability of Treatment Weighting \\
\hline MEPS & Medical Expenditure Panel survey \\
\hline MCS & Mental Component Summary \\
\hline PCS & Physical Component Summary \\
\hline PDC & Proportion of Days Covered \\
\hline PPO & Preferred Provider Organization \\
\hline $\mathrm{ROC}$ & Receiver Operating Characteristic \\
\hline SD & Standard Deviation \\
\hline SE & Standard Error \\
\hline $\mathrm{SF}-12$ & Short-Form Health Survey \\
\hline SIG & Significance \\
\hline TNFi & Tumor Necrosis Factor Inhibitor \\
\hline US & United States \\
\hline USD & United States Dollars \\
\hline $\mathrm{Wt}$ & Weighted \\
\hline
\end{tabular}




\section{CHAPTER 1}

\section{Introduction}

\subsection{Background and Significance}

\section{Epidemiology of RA}

Rheumatoid Arthritis (RA) is the most prevalent immune mediated arthritis affecting nearly 1.5 million adults in the United States (US) ${ }^{1}$. The prevalence of RA in the U.S. general population aged 18 years or older is $0.72 \%{ }^{1}$. Among the commercially insured adult population, the prevalence of RA ranges from $0.41 \%$ to $0.54 \%^{2}$. RA disproportionately affects women with more than $75 \%$ of RA patients being women ${ }^{3}$. The lifetime risk of developing RA is $4 \%$ for women and $3 \%$ for men in the US ${ }^{4}$.

The clinical burden can be measured by mortality, disease activity, pain, fatigue, and the high prevalence and incidence of other physical and mental health conditions ${ }^{5-7}$. Other clinical outcomes such as functional status are worse in RA patients as compared to the general population as well as those with osteoarthiritis, psoriatic arthritis and ankylosing spondylitis ${ }^{7}$.

The inflammatory and immune pathophysiology of RA can also lead to the high prevalence of other chronic conditions ${ }^{8}$. Other chronic physical and mental health conditions that are most prevalent among patients with RA include hypertension (47.5\%), mental health conditions (36\%), pulmonary diseases $(25.5 \%)$, endocrine problems $(20.3 \%)$, cardiovascular diseases $(18.8 \%)$, and gastrointestinal disorders $(15.4 \%)^{9}$. A typical patient with RA has 2 additional chronic conditions and the burden of chronic conditions increase with increase in the severity of RA, duration of RA and age ${ }^{10}$. The presence of other chronic conditions can increase the risk of mortality and morbidity among RA patients relative to the general population ${ }^{11}$.

\section{Depression in RA}


Of all the chronic conditions that can co-exist or develop in RA patients, depressive disorders deserve special attention because of the special pathophysiology between RA and depression.

\section{Link between RA, Inflammation and Depression}

Patients with RA may have high prevalence and incidence of depression because of the heightened level of systemic inflammation underlying the pathogenesis of RA. One study done in 218 RA outpatients, demonstrated a significant association between depression severity and level of inflammatory marker C-reactive protein (CRP) even after adjustment of RA severity, indicating the potential role of inflammation in the pathogenesis of depression ${ }^{12}$. In addition, some studies have also shown that administration of pro-inflammatory cytokines leads to the development of depressive symptoms among individuals without pre-existing depression ${ }^{13,14}$.

\section{Prevalent Depression in RA}

A meta-analysis of 72 studies that included 13,189 RA patients, reported that the prevalence of major depressive disorders in RA patients was $16.8 \%$ (versus $6.7 \%$ in general population) ${ }^{15}$. The same meta-analysis also reported the presence of depressive symptoms in $38.8 \%$ of RA patients measured using Patient Health Questionnaire (PHQ-9) and 34.2\% of RA patients measured using Hospital Anxiety and Depression Scale (HADS) ${ }^{15}$. An inverse relationship was found between the presence of depression and age among RA patients indicating a higher prevalence of depression among the younger as compared elderly population with $\mathrm{RA}^{15}$.

\section{Incident Depression in RA}

One longitudinal study conducted on 4,187 RA patients in the U.K found that almost $30 \%$ of RA

patients developed depression within 5 years of RA onset, ${ }^{16}$. The incidence of depression in RA 
is $74 \%$ higher than the general population ${ }^{17}$. The lifetime risk of developing depression in RA is 2 times higher in RA patients as compared to the general population ${ }^{18}$.

\section{Bidirectional Relationship between RA and Depression}

A bidirectional relationship between RA and depression has been reported; RA increases the risk of depression and the onset of depression may also predict increasing severity, pain and disease activity in $\mathrm{RA}^{19,20}$. A recent study found that the onset of RA increased the hazards of depression onset by $69 \%$ and the onset of depression increased the hazards of RA by $65 \%$, even after adjusting for age and $\operatorname{sex}^{20}$. Another cohort study also reported similar bidirectional relationship between depression and RA even after adjusting for the genetic risk for depression and $\mathrm{RA}^{21}$.

\section{Disease Burden of Depression in RA}

\section{Clinical Burden: Mortality and Morbidity}

As compared to RA patients without depression, RA patients with depression have poorer survival. For example, one study on 1290 outpatients with RA found that prevalent depression increased mortality risk by more than 2 folds in RA patients even after adjusting for sociodemographic factors, comorbidities and RA severity ${ }^{22}$. In particular, the risk of cardiovascular morbidities such as myocardial infarction and burden of atherosclerosis is significantly increased among RA patients with depression as compared RA patients without

depression ${ }^{23,24}$. A retrospective cohort study of 15,634 RA patients, revealed that the presence of depression in RA was associated with a $40 \%$ increase in myocardial infarction even after controlling for sociodemographic and other risk factors ${ }^{23}$. Another longitudinal cohort study on 195 RA patients, depressive symptoms were associated with 3.4 times increase in atherosclerotic 
burden ${ }^{24}$. Strong associations with depression and suicidal ideation in RA patients is also well documented in literature ${ }^{25-27}$.

Depression can negatively affect clinical remission and disease activity in RA patients ${ }^{28-}$

${ }^{30}$. Depressive symptoms at baseline in RA patients has been reported as an independent predictor of disease activity in RA patients even after adjusting for the demographic factors, RA duration and severity ${ }^{31}$. A descriptive study done on 159 RA patients in the United Kingdom (UK) reported that persistent depression was associated with RA disease activity ${ }^{32}$. Presence of depression can lead to a significant increase in RA severity. Matcham et.al., measured increase in Disease Activity Score in 28 joints (DAS28) and reported that depression significantly reduces the likelihood of clinical remission of $\mathrm{RA}^{28}$.

Most RA patients experience pain and fatigue due to the disease ${ }^{33}$. The pain severity can be exacerbated by the presence of depression and increase in pain severity among RA patients ${ }^{34}$. A systemic review of 29 studies on RA patients found that depression was the most consistent predictor of fatigue in RA patients.

\section{Humanistic Burden}

RA by itself exerts a negative influence on all aspects of health related quality of life (HRQOL) such as physical functioning, limitations due to physical function, and bodily pain ${ }^{7,35}$. One recent study in 1,324 RA patients, showed that with the availability of improved treatment options for RA, although the disease activity in RA has reduced by half from 1996 to 2014, disability scores and quality of life outcomes remained unchanged ${ }^{36}$. It is now widely recognised that RA affects several aspects of an individual's life such as work capacity, financial status, social relationships, physical and psychological well-being ${ }^{6,37}$. For example, one panel study on 548 RA patients in the U.S. reported that $68.4 \%$ of RA patients had problems with at least one 
activity of daily living; while $91.4 \%$ of RA patients had problems with at least one committed activities of living such as paid work and household responsibilities; and $92.5 \%$ of RA patients had problems with at least one discretionary activities such as socializing, leisure activities and exercise $^{38}$. In another longitudinal study on 5,384 RA patients done in the U.S., one-fourth, onethird and half of RA patients respectively experienced permanent work disability in 3 years, 10 years and 25 years of disease duration ${ }^{39}$.

The present of depression significantly adds to the humanistic burden in RA patients; it worsens the HRQoL ${ }^{40,41}$ in both physical and mental health domains ( ${ }^{42}$. One longitudinal study on 307 RA patients showed that depressive symptoms were a more important predictor of HRQoL than disease activity or radiographic damage in RA ${ }^{43}$.

\section{Economic Burden}

The economic burden associated with RA is substantial due to the high costs of medical care and productivity loss due to absenteeism and loss of employment. In the US, the aggregated total incremental direct medical costs associated with RA was $\$ 22.3$ billion (2008 USD) ${ }^{44}$. The national indirect costs associated with RA due to missed work days were $\$ 252$ million annually 45. A review of studies on the indirect economic burdent of RA concluded that RA was associated with loss of work productiivty ${ }^{46}$.

Depression can exacerbate the direct and indirect economic burden in RA patients beyond that required for depression treatment. One study has reported that the presence of depression was associated with increases in RA-related hospitalizations and outpatient visits ${ }^{47}$. There could be additional economic burden of depression in RA due to medication nonadherence. For example, RA patients with depression are less likely to be adherent to treatment regimens ${ }^{48}$. Such non-adherence may be associated with considerable increase in disability, 
reduced quality of life, and poor clinical remission, which can lead to higher economic burden among adults with RA and depression compared to RA patients without depression.

However, no recent study in the US has done a comprehensive analysis of the incremental economic, clinical and humanistic burden of depression among RA patients. In fact, an expert review on depression among adults with arthritis concluded that more research is needed in this area ${ }^{49}$.

\section{Importance of RA and Depression in working-age adults}

Such studies are especially important among individuals of working-age for several reasons: the prevalence of depression in this population is higher than older adults; the onset of RA typically begins during the most productive mid-life years, between the ages 30 and 60 years ${ }^{50}$. Similarly, depression also affects individuals during the prime working years of lives, between the ages 24 and $44{ }^{51}$. Both RA and depression are associated with substantial disability. One-third of RA patients experience permanent work disability in 3 years, 10 years and 25 years of disease duration ${ }^{39}$. Depression is also considered as a leading cause of disability among working age adults and is associated with substantially reduced work performance due to missed work days, presenteeism and premature loss of employment and decrease in income ${ }^{52,53}$. Such disabiilties can lead to high productivity losses. For example, nearly $60 \%$ of adults with RA may be working at the time of onset (with RA may be employed ${ }^{54}$. A systematic review of literature on productivity losses in RA patients concluded that although methodologies and costs vary across studies the loss represent real cost to both employers and RA patients ${ }^{46}$.

Therefore, it is important to have a more comprehensive understanding of the effect of depression on clinical outcomes (e.g. pain-related interference, treatment response to antirheumatic therapies), humanistic outcomes (e.g. health related quality of life), economic 
outcomes (e.g. medical costs, lost wages due to missed work) among working-age adults with RA. This leads to the rationale for Aim1, which estimated the incremental clinical burden (pain related interference with normal work), humanistic burden (health related quality of life) and direct and indirect economic burden (healthcare costs, missed work days, lost wages due to missed work days) associated with depression among working-age RA patients.

\section{Depression and Treatment of RA}

Since 1997, the treatment of RA has undergone vast changes with the introduction of the biological response modifiers, also called biological disease-modifying anti-rheumatic drugs (bDMARDs) ${ }^{55}$. These are genetically engineered large protein molecules that block the activity of inflammatory cytokines such as TNF- $\alpha$, Interleukin-1 (IL-1), Interleulin-6 (IL-6) or cell surface receptor molecules such as Janus Kinase ${ }^{56}$. Currently FDA approved biologics for the treatment of RA include five TNF inhibitors (Etanercept, Infliximab, Adalimumab, Certolizumab pegol, Golimumab), IL-1 inhibitor (Anakinra), T-cell co-stimulation blocker (Abatacept), B cell targeted therapy (Rituximab), IL-6 inhibitor (Tocilizumab) and Janus Kinase inhibitors (Toficitinib)(Singh, Saag et al. 2016). Biologic agents are highly effective in slowing the rate of long-term structural damage, reducing RA related symptoms, improving physical functioning, quality of life, work and productivity and reducing comorbidities ${ }^{56}$. Furthermore, the clinical response to biologics are usually rapid and takes place within days or few weeks of initiating therapy $^{55}$.

\section{Depression and Tumor Nectrosi Factor Inhibitor Therapy in RA}

Tumor Necrosis Factor inhibitor (TNFi) biologics are recommended as first line biologic therapy for RA patients with moderate to severe disease who failed therapy with conventional DMARDS such as methotrexate, sulfasalazine and hydroxychloroquine ${ }^{57}$. Several randomized 
controlled clinical trials have proven the high efficacy of TNFi in RA patients after treatment failure with conventional DMARDs such as Methotrexate ${ }^{58-60}$. A recent analysis of US prescribing patterns reported an increase in biologic use from $3 \%$ of patients in 1999 to $26 \%$ in $2006^{61}$. The prescribing rate of biologics for RA increased by nearly 9 folds from $3 \%$ of RA patients in 1999 to $26 \%$ of RA patients in 2006 . A study on commercially insured RA patients have found that nearly one-quarter of patients with incident RA are prescribed TNFi therapy within 1.75 years after diagnosis ${ }^{62}$.

However, very few studies have evaluated the effect of depression on treatment response to particular antirheumatic therapies such as TNFi. One longitudinal cohort study on $166 \mathrm{RA}$ patients treated with TNFi in the UK showed that depressive symptoms were significantly associated with the discontinuation of TNFi therapy ${ }^{48}$. However, these studies assessed depression as one of the independent variables within a multivariate framework and did not evaluate the extent to which each of the individual level variables such as demographics and clinical factors in RA patients contributes to the difference in treatment response to TNFi by depression status.

This leads to the rationale of the second aim, which evaluated the effect of depression on treatment response to TNFi among working age RA patients who are cared for in routine clinical practice. In addition, a post-regression decomposition approach was adopted to explain factors that may contribute to difference in the treatment response to TNFi by depression status.

\section{Tumor Nectrosi Factor Inhibitor Therapy in RA and Risk of Depression}

There has been a growing interest in examining whether anti-inflammatory therapies may have beneficial effect in reducing depression among all individuals ${ }^{63}$ because of the role of inflammation in depression and many medical conditions ${ }^{64}$ and specifically in patients with 
$\mathrm{RA}^{65}$. A meta-analysis of 14 randomized-controlled trials (RCTs) including 6,262 participants revealed that non-steroidal anti-inflammatory drugs (NSAIDS) are significantly more effective as compared to placebo in reducing depressive symptoms ${ }^{66}$. Other powerful anti-inflammatory drugs such as inflammatory cytokine inhibitor biologic therapies, including tumor necrosis factor inhibitor (TNFi) are also being studied for their potential anti-depressant effect. Another recent meta-analysis of 7 RCTs including 2,370 participants reported a significantly higher antidepressant effect of biologic therapies such as TNFi as compared to placebo ${ }^{65}$. These observations have strengthened the hypothesis that inflammation plays a crucial role in the pathogenesis of depression and suppression of inflammation with powerful anti-inflammatory drugs such as TNFi in patients suffering from inflammatory arthritis such as RA may have a potential beneficial effect in reducing depression.

Findings from the above mentioned studies sugest that anti-inflammatory effect of TNFi therapy may have spillover effects and may reduce the risk of depression in RA patients ${ }^{67,68}$. However, not all RA patients respond to TNFi therapy. Infact, in real-world clinical practice, only one-third of RA patients respond to TNFi therapy(Curtis, Schabert et al. 2014). Therefore, the beneficial effect of TNFi on depression may be limited to RA patients who respond to TNFi therapy. To date, no real world clinical study has evaluated the effect of treatment response to TNFi on the risk of developing depression in RA patients.

This leads to the rationale of the aim 3, which examined the effect of treatment response to TNFi therapy on the risk of developing depression among working age commercially insured RA patients 


\subsection{Innovation}

a) Comprehensive analysis of the illness burden of depression among a nationally representative sample of working-age RA patients with depression: This is the first study to conduct a comprehensive analysis of the clinical, humanistic and economic burden associated with depression among working-age adults with RA.

b) Use of a novel claim based algorithm to measure treatment response to TNFi: The algorithm to measure treatment response to biologic drugs in RA developed by Curtis et al. using claims data has been validated and accurately captures the clinical response to TNFi. This novel algorithm enables us to assess treatment response to TNF inhibitors among RA patients who are cared for in real world practice setting utilizing data that are readily available from claims. Till date, this algorithm has been only used for descriptive analysis of the comparative effectiveness of various biologics in RA. Our study is the first study to use this algorithm to model treatment response while controlling for various clinical and demographic factors.

c) Application of econometric techniques to identify difference in characteristics between patients with and without depression that explain disparity in treatment response between the two groups: This is the first study to examine the contribution of various individual characteristics of RA patients to the observed gap in the treatment response rate between those with depression versus those without depression from a population based perspective.

d) Protective Effects of TNF inhibitors in reducing the risk of depression in RA patients: This is the first observational study to use a nation-wide database of commercially insured working-age RA patients to evaluate the association between response to TNFi and risk of newly-diagnosed depression. 
e) Use of a nationally representative data of commercially insured RA patients: One of the main advantages of using the IMS Pharmetrics claims data for this study is the availability of a large, diverse, nationally representative population of working age commercially insured RA patients.

\subsection{Specific Aims}

AIM 1: Evaluate the incremental illness burden associated with depression among workingage $R A$ patients. The illness burden domains are: Clincial - pain related interference with normal work; Humanistic - health related quality of life and Economic burden -healthcare costs, missed work days, lost wages due to missed work days.

Hypothesis: RA patients with depression will have a higher clinical, humanistic and economic burden as compared to RA patients without depression.

AIM 2: Examine the association between depression and treatment response to TNFi among working age commercially insured $R A$ patients.

Hypothesis: RA patients with depression will be less likely to respond to TNFi therapy as compared to RA patients without depression.

AIM 3: Investigate the effect of treatment response to TNFi therapy on the risk of newly diagnosed depression among working-age commercially insured RA patients.

Hypothesis: RA patients who respond to TNFi therapy will be less likely to develop depression as compared to RA patients who does not respond to TNFi therapy.

\subsection{Approach}

\section{Conceptual Framework}

Aim 1 and Aim 2: The conceptual framework for this study is adapted from the Andersen's Expanded Behavioral Model ${ }^{69}$ which posits that healthcare utilization by an 
individual is a function of predisposing factors (e.g. age, sex ), enabling factors (e.g. the type of plan, the calendar year of TNF inhibitor initiation), need factors (e.g. chronic conditions, RA severity, baseline health service utilization) and external environment factors (e.g. geographical region). As the effectiveness construct was operationalized based on prescription drug utilization, the Andersen's Behavioral Model was an appropriate conceptual model for selecting the independent variables in our study. The predisposing factors represents the individual characteristics that predict the propensity for use of healthcare services. The enabling factors represent the factors that enable an individual to gain access to healthcare services. The need factors are those characteristics that define an individual's health status. The external environment represent the environmental and geographical factors that influence an individual's health service utilization.

Aim 3: The conceptual framework for this study was adapted from the determinants of a health and chronic disease model proposed by Wilkinson and Marmot ${ }^{70}$. This model delineates the relationship between community resources, access to care variables, individual physical make-up, biological risk factors, and treatment factors that affect the incidence of chronic conditions.

\section{Data Sources}

To accomplish the study objectives, we used data from multiple sources. To estimate the incremental clinical, humanistic, and economic burden of depression in RA patients (aim 1), we selected the nationally representative survey of the US civilian non-institutionalized households, namely the Medical Expenditure Panel survey (MEPS). The MEPS collects information on demographic characteristics, medical conditions, health status, health-related quality of life, utilization of health care services, charges and payments, access to care, health insurance 
coverage, income, education, employment and missed work days of all members in the selected households.

To analyze the interaction between depression and treatment response (aims 2 and 3) we used IMS PharMetrics Plus claims data for the period October 1, 2009 to September 30, 2015. PharMetrics Plus is an integrated claims database that includes enrollment, medical and pharmacy claims information for more than 95 million enrollees of commercial plans across the US. The data includes the records of $90 \%$ of hospitals, $80 \%$ of doctors and $85 \%$ of large companies in the US. This database also records enrollee's demographics (year of birth, gender, geographic region), plan type (health maintenance organization, preferred provider organization), payer type (commercial, self-insured), prescription drug information and considered nationally representative of commercially insured individuals less than 65 years of age in the US. 


\section{CHAPTER 2}

\section{Burden of Depression among Working-Age Adults with Rheumatoid Arthritis}

\subsection{Abstract}

Objective: This study estimated the excess clinical, humanistic, and economic burden associated with depression among working-age adults with Rheumatoid Arthritis (RA). Methods: A retrospective cross-sectional study was conducted among working-age (18 to 64 years) RA patients with depression( $\mathrm{N}=647)$ and without depression $(\mathrm{N}=2,015)$ using data from the nationally representative Medical Expenditure Panel Survey for the years 2009, 2011, 2013 and 2015. The clinical outcome was: level of pain interference with normal work and any functional limitations; humanistic outcomes were: health-related quality of life (HRQoL) measures from the 12-item short-form health survey(SF-12-V2); and economic outcomes were: direct total annual healthcare expenditures, out-of-pocket spending, and burden, unemployment, the number of annual missed work days and lost wages due to missed work days. Counter-factual recycled prediction method was used to assess the incremental burden associated with depression. Results: Overall, 25.8\% had depression. In adjusted analyses, adults with RA and depression compared to those without depression were significantly more likely to have pain interference with normal work (severe pain: $\mathrm{AOR}=2.22 ; 95 \% \mathrm{CI}=1.55,3.18$ ), any functional limitations $(\mathrm{AOR}=2.17 ; 95 \% \mathrm{CI}=1.61,2.94)$, lower mental health HRQoL scores. Adults with RA and depression had significantly higher annual healthcare expenditures $(\$ 14,752$ vs. 10,541, p $<.001$ ), and out-of-pocket spending burden. Adults with RA were more likely to be unemployed and among employed adults, those with depression had a significantly higher number of missed work days annually and higher lost annual wages due to missed work days. Conclusions: This study highlights the importance of effectively managing depression in routine clinical practice of 
RA patients to reduce pain, any functional limitations, improve quality of life, lower direct and indirect healthcare costs.

\subsection{Introduction}

Rheumatoid Arthritis (RA) is one of the most debilitating chronic conditions, with the onset often occurring during the prime working years of lives, between the ages 20 and 40 years 50. Individuals with RA experience substantial pain and RA causes permanent work disability in more than one-third of affected patients within 10 years of onset ${ }^{39}$. Such pain and disabilities associated with RA may contribute a higher prevalence of depression in individuals with RA compared to healthy controls ${ }^{71}$. An expert review of depression in arthritis reported that the prevalence of depression in adults with RA can be as high as $66.2 \%{ }^{49}$. A systematic review and meta-analysis of 72 studies estimated the prevalence rate to be $16.8 \%{ }^{72}$.

The disease burden of depression in RA can be substantial because depression can worsen survival ${ }^{73}$ and increase morbidity in terms of disability, health-related quality of life (HRQoL), RA disease activity ${ }^{28}$ and pain ${ }^{12}$. Depression in RA can also increase healthcare resource utilization ${ }^{47,74}$, which can lead to high health care expenditures for both insurance payers and patients and families. As depression in RA can aggravate disability, an individual may also suffer economic losses due to work inability or even experience economic losses due to missed work days. Although not specific to RA, one study estimated that 6.9 million workingage adults reported arthritis-attributable work limitation ${ }^{75}$. One can speculate that depression can worsen the work-limitation because the presence of depression along with any chronic physical condition more than doubles the likelihood of work absenteeism as compared to the presence of any chronic physical condition without depression ${ }^{76}$ 
However, to date, no published study in the US has done a comprehensive analysis of the humanistic and economic burden associated with depression among RA patients, particularly among working-age adults. In fact, a recent review highlighted the significant knowledge gap in estimating the disease burden of depression in adults with arthritis ${ }^{49}$. Although there has been a handful of studies on the association between depression and HRQoL among adults with RA, most of these studies have been conducted outside the US ${ }^{77,78}$ or only among women in a specific setting ${ }^{79}$. One US study used a cross-sectional design and examined the relationship between depression and disability, HRQOL in the US with data from 2011 he 2011 Behavioral Risk Factor Surveillance System ${ }^{80}$. However, this study included all forms of arthritis and did not focus on RA. Again, only one study using 2006 commercial claims data in the U.S., found that RA patients with depression had a significantly higher adjusted annual healthcare costs as compared to RA patients without depression $(\$ 12,225 \text { vs. } \$ 11,404)^{81}$. However, this study was based on commercially insured RA patients and may not be representative of the U.S. national population. Furthermore, this commercial insurance data did not include certain patient characteristics which are important confounders of healthcare costs such as race, education and income level.

Therefore, the objective of this study is to examine the incremental burden of depression on the clinical, humanistic and economic outcomes among working-age adults with RA.

\subsection{Methods}

\section{Study Design}

A retrospective cross-sectional study design with data from a nationally representative sample of working-age adults (18-64 years) was used.

\section{Data Source}


We used data from the Medical Expenditure Panel Survey (MEPS), an annual household survey of the non-institutionalized civilian population in the U.S. Information on demographic characteristics, medical conditions, health status, utilization of health care services, charges and payments, access to care, health insurance coverage, income, education, employment and missed workdays of the participants in the survey were extracted from the household component of MEPS. We pooled four years of data $(2009,2011,2013$, and 2015) to have sufficient sample size, and used data from alternate years to avoid including two observations per individual. Furthermore, a question related to the type of arthritis was available in these years. Indeed, MEPS recommends pooling of data to increase sample size and it is a common practice in published literature with MEPS data ${ }^{82}$.

\section{Study Sample}

The study sample $(\mathrm{N}=2,662)$ consisted of working-age (18-64 years) who were diagnosed with RA and who were alive during the study period (2009, 2011, 2013, and 2015). RA was identified from the priority condition enumeration section. In this section, respondents were queried whether an individual in the household has ever been told by a doctor or other health professional that she/he had arthritis and type of arthritis (RA versus osteoarthritis). We also identified RA from medical condition file with the clinical classification code (202). Medical conditions were reported by the respondents if they sought treatment for the condition, or if the condition resulted in disability, or if the condition was bothersome. The responses were recorded as texts, and these texts were translated into International Classification of Diseases, 9th Edition, Clinical Modification (ICD-9-CM) codes by professional coders. In addition, MEPS data provides clinical classification codes, which are aggregated ICD-9-CM codes into clinically 
meaningful categories that group similar conditions (Agency for Healthcare Research and Quality).

\section{Conceptual framework}

The conceptual framework for this study was adapted from the Andersen's Expanded Behavioral Model which posits that health services utilization and outcomes of an individual is a function of predisposing factors (e.g., age, sex, and race), enabling factors (e.g. marital status, education and poverty status), need factors (e.g. chronic conditions, health status), and personal health practices (e.g. physical activity, obesity and smoking $)^{69}$.

\section{Measures}

\section{Clinical Outcomes:}

Pain Interference with Normal Activities: Based on a self-administered single-item question, pain interference with normal activities during the past four weeks among the household respondents was measured. The answers were recorded on a 5-point Likert scale during the past 4 weeks. In MEPS, pain was reported on a 5-point scale: 1) Not at all, 2) A little bit, 3) Moderately, 4) Quite a bit, and 5) extremely. For purposes of this study we group pain categories as follows: 1) Not at all/little bit; 2) Moderate; 3) Severe (quite a bit/extremely). Selfreported pain from MEPS has been used in published literature to estimate the cost of pain ${ }^{83}$. There were 42 individuals (5.9\%) in the depression group and 140 individuals $(6.6 \%)$ in the no depression group with missing data on pain inference variable. These individuals were not included in the analysis.

Any Functional Limitations: This variable summarizes whether an individual had any limitations in instrumental activities of daily living (IADL) (e.g. shopping, cooking, using phone, paying bills, taking medications, driving, doing laundry, or going shopping), activities of daily 
living (ADL) (e.g. bathing, dressing, grooming, mouthcare, toileting, eating), functional (walking, climbing stairs, grasping objects, reaching overhead, lifting, bending or stooping, or standing for long periods of time) or activity limitations (work, housework, or school).

\section{Humanistic Outcome: Health-related Quality of Life}

HRQoL was measured by the generic Short-Form-12 Version 2 (SF12-V2) summary scores. The SF12-V2 is a generic multipurpose survey with 12 questions, which encompass eight domains (role physical, role emotional, physical function, social function, mental health, vitality, pain, and general health). These questions are designed to provide summary measures of overall HRQoL of an individual. The Mental Component Summary (MCS) score was derived from the responses to the items in the domains: vitality, social functioning, role-emotional (limitations in work and daily activities because of emotional problems), and mental health. The Physical

Component Summary (PCS) score was derived from the responses to the items in the domains: physical functioning, role-physical, bodily pain, and general health. Both MCS and PCS scores ranged from 0 to 100, with higher scores representing better self-reported health and better HRQoL related to mental or physical health ${ }^{84}$.

\section{Economic Outcomes: Direct Healthcare Expenditures}

Total healthcare expenditures: In the MEPS, expenditures are defined as the sum of direct payments for care provided during the year. The direct payments include twelve sources of payment categories such as out-of-pocket by patient or families, Medicare, Medicaid, Private insurance, Veteran Administration, worker's compensation, and others. Total annual per person healthcare expenditures were calculated as the sum of inpatient, outpatient, emergency, dental, home health, vision, prescription drugs and other medical supplies. All expenditures were inflation adjusted to 2015 US dollars (USD) using consumer price index for medical services 
from the bureau of medical services.

Total out-of-pocket spending burden by patients and families: We also estimated the total out-of-pocket spending on healthcare by the respondent and/or family. These included annual deductibles, copayment, and coinsurance for services and payment for services that were not covered by health insurance. We calculated out-of-pocket spending burden as the ratio of out-ofpocket healthcare expenditures to personal income ${ }^{85}$, which varied from zero to 100 . Based on published literature, we defined spending $10 \%$ or more of personal income on health care as high out-of-pocket spending burden ${ }^{86}$.

\section{Economic Outcomes: Indirect Healthcare Burden}

Unemployment (i.e. labor market outcome). In the MEPS, employment section covers questions about each person's employment or self-employment status. Based on these questions, we classified individuals were currently unemployed.

Missed work days were measured whether individuals lost a half-day or more from work because of illness, injury, or mental or emotional problems during the year and how many workdays were lost. This was calculated only for employed adults.

Lost wages for each individual were calculated by multiplying missed work days with an average daily wage of each individual. All wages were adjusted to 2015 general consumer inflation rates derived from the bureau of labor statistics.

\section{$\underline{\text { Key Explanatory Variable }}$}

Depression (yes/no): Depression was identified based on the clinical classification code “657”, which included both depressive disorders and bipolar disorders.

\section{Other Explanatory Variables}

Predisposing characteristics were sex (male, female), race/ethnicity (Whites, African- 
American, other racial minorities) and age in years (18-39, 40-49 and 50-64). Enabling factors comprised marital status (married, widow, separated/divorced, never married), family poverty status (not poor, poor), health insurance status (public, private) and usual source of care (yes, no). Need Factors included having a chronic condition other than RA from a list of eight conditions: asthma, cancer, chronic obstructive pulmonary disease, diabetes, heart disease, hypertension, stroke, and thyroid), anxiety, perceived physical health status (excellent/very good, good and fair/poor) and perceived mental health status (excellent/very good, good and fair/poor). Personal health practice factors included obesity (obese and not obese), smoking status (current smoker, other, missing) and exercise ("yes" and "no" exercise).

\section{Statistical Analyses}

A variety of statistical analyses were used based on the measurement of the outcome variables. The unadjusted relationships between the presence of depression and categorical variables and outcomes (pain interference with activities, employment, and OOP burden) were assessed with chi-square tests. Unadjusted differences in continuous outcomes (PCS, MCS, allcause healthcare expenditures, out-of-pocket spending by the patients and their families) by depression were tested with t-tests. Multinomial logistic regression was used to analyze the association between depression and pain-related interference with normal work after adjusting for the predisposing, enabling, need and external environment characteristics. Logistic regression was used to analyze the association between depression and binary categorical variables (e.g. any limitations, unemployment, out-of-pocket spending burden) after adjusting for covariates. Adjusted models for continuous outcomes (expenditures, out-of-pocket expenditures, and lost wages) consisted of Generalized Linear Models (GLM). GLM is flexible and can handle categorical outcomes, continuous outcomes, and count-data with the appropriate distribution 
family and a link function. For categorical outcomes, we conducted logistic regression. For count-data (e.g. the number of missed work days) we used negative binomial regression.

Counterfactual prediction technique (Recycled Prediction): We used counterfactual recycled prediction, an approach that is gaining attention ${ }^{87,88}$ to estimate excess total healthcare expenditures, prescription expenditures, missed work days and lost wages attributable to depression among working-age adults with rheumatoid arthritis. The recycled prediction technique is a preferred approach because it adjusts for differences in characteristics between the depression and no depression group by creating counterfactual scenarios. In all recycled prediction models, confidence intervals were obtained using 2000 bootstrap replications using the percentile method. To account for the complex design of MEPS, we conducted all analyses using the survey procedures in Statistical Analysis Software (SAS) version 9.3, Cary, NC, USA and the survey design features with STATA 14 . As we pooled four years, to get annualized weighted numbers, we divided the weights by four, recommended by the MEPS investigators ${ }^{89}$ and used in the published literature ${ }^{90}$.

\subsection{Results}

\section{Description of the study sample}

Majority of the study sample was female (64\%), white (63\%), aged between 50 and 64 years $(61 \%)$ and had multi-morbidity (72\%). Only $26 \%$ of individuals who perceived themselves having excellent or very good physical health and $44.8 \%$ reported having excellent or very good mental health (See Appendix 7.1).

Overall, 25.8\% of adults with RA reported depression (Table 2.1). We observed significant differences in the rate of depression by predisposing, enabling, need factors, and personal health practices except for age, education, and region. For example, female adults with 
RA reported a significantly higher rate of depression than their male counterparts ( $29.6 \%$ vs. 19.1\%). The higher rate of depression was also observed among individuals with multimorbidity ( $29.1 \%$ vs. $17.4 \%)$. A higher percentage of those who perceived themselves to be poor/fair physical health reported depression compared to those in excellent or very good health (38.0\% vs. $12.4 \%)$.

\section{Clinical Outcomes}

Pain Interference with Normal Activities

A higher percentage of adults with RA and depression reported severe pain interfering with work or other normal activities compared to those with RA and no depression (54.8\% vs. 30.8\%) (Table 2.2). After adjusting for predisposing, enabling, need, personal health practices, and external environment factors, adults with depression were 2.2 times as likely to report severe pain interference with normal work activities than those without depression $(\mathrm{AOR}=2.22 ; 95 \%$ $\mathrm{CI}=1.55,3.18)$.

\section{Any Functional Limitations}

A significantly higher percentage of adults with RA and depression reported any limitations compared to those with RA and no depression (79\% vs. 51.1\%) (Table 2.2). After adjusting or covariates adults with RA and depression were more than 2 times as likely to report

any functional limitations $(\mathrm{AOR}=2.24 ; 95 \% \mathrm{CI}=1.62,3.10)$ compared to adults with $\mathrm{RA}$ and no depression.

\section{Humanistic Outcomes}

Adults with RA and depression reported significantly lower HRQoL scores in both physical component summary score ( $35.1 \mathrm{vs} 40.2, \mathrm{p}<.001)$ and mental component summary score (37.2 vs 48.7, p<.001) compared to adults with RA without depression (Table 2.3). In 
adjusted analyses, a significant difference was observed only in the mental domain of the HRQoL; the presence of depression was associated with a decrement of 8.72 in MCS scores (Table 2.3). The counterfactual predictions yielded similar differences in MCS (37.19 in adults with depression vs. 45.91 in adults without depression, $\mathrm{p}<.001)$. The relationship between depression and PCS scores became insignificant after adjustment for the presence of multiple chronic conditions.

\section{Economic Outcomes}

\section{Direct Total Healthcare Expenditures}

In unadjusted analysis, adults with RA and depression had significantly higher annual healthcare expenditures $(\$ 17,941$ vs. $\$ 10,064 \mathrm{p}<.001)$. In the adjusted GLM with gamma distribution and log-link, we found that depression was associated with greater total healthcare expenditures compared to those without depression (Beta $=0.34, \mathrm{SE}=0.08)$. When converted to original dollars this represented $\$ 14,752$ for those with depression and $\$ 10,541$ for those without depression (Table 2.4). Estimates from counter-factual recycled prediction revealed that depression was associated with an excess of $\$ 4,212$ total healthcare expenditures with $95 \% \mathrm{CI}=$ $\$ 4,114, \$ 4,318$.

In unadjusted analysis, patients/families in the RA + depression group spent significantly higher amounts out-of-pocket on health care compared to the RA + no depression group $(\$ 1,443$ vs. $\$ 1,052, \mathrm{p}<.001)$. In the adjusted GLM with gamma distribution and log-link, we found that depression was associated with greater total out-of-pocket healthcare spending compared to those without depression $($ Beta $=0.23, \mathrm{SE}=0.06)$. When converted to original dollars this represented $\$ 1,232$ for those with depression and $\$ 979$ for those without depression (Table 4). 
Estimates from counter-factual recycled prediction revealed that depression was associated with an excess of $\$ 253$ with $95 \% \mathrm{CI}=\$ 247,260$.

When high out-of-pocket spending burden was measured as spending greater than $10 \%$ of income on healthcare, we found that $30.7 \%$ of adults with depression and $21.3 \%$ of adults without depression had high out-of-pocket spending burden. After adjusting for other factors, adults with depression were significantly more likely to have high out-of-pocket spending burden $(\mathrm{AOR}=1.34 ; 95 \% \mathrm{CI}=1.01,1.79)$.

\section{Indirect Economic Burden}

\section{Labor market outcome (Unemployment), missed work-days and lost wages}

Presence of depression was significantly associated with unemployment among adults with RA; $64.1 \%$ of adults with depression were unemployed compared to $40.1 \%$ adults without depression. Even after controlling for other factors mentioned in the methods section, adults with RA and depression were 1.55 times as likely as those without depression to be unemployed $(\mathrm{AOR}=1.55 ; 95 \% \mathrm{CI}=1.14,2.10)$. Among employed adults, those with depression had significantly higher number of missed work days annually (9 vs. $6, \mathrm{p}<0.05)$ and higher lost wages ( $\$ 813$ vs. $\$ 571, \mathrm{p}<0.05$ ) due to missed work (Table 2.4$)$. We obtained similar results with counterfactual recycled predictions.

\subsection{Discussion}

In this study using a nationally representative sample of community-dwelling US adults, one in four working-age adults with RA reported depression. This rate is considerably higher compared to the $6.8 \%$ rate of depression in the general population in the U.S. ${ }^{51}$ and higher than the pooled depression rate of $16.8 \%$ reported by Matcham and collegues ${ }^{72}$. The difference in 
rate can be explained by the differences in time period, study population and instruments that were used to identify depression.

Our study findings indicated the substantial additional clinical burden imposed by depression in working-age adults with RA. These findings have implications for co-management of depression and RA. Although not specific to RA, a randomized clinical trial of 1,001 patients with concurrent depression and arthritis and seeking care from 18 primary care clinics ${ }^{91}$ suggested that collaborative depression care not only reduced depressive symptoms but also improved arthritis related outcomes, such as decreasing pain and functional limitations. There is some evidence that disease-modifying drugs used to treat RA can have spill-over effects in reducing depressive symptoms. For example, depression levels decreased significantly following commencement and continuity of rituximab, a B cell-directed therapy, among individuals with $\mathrm{RA}^{92}$. Therefore, future studies need to systematically evaluate whether anti-rheumatic treatment among individuals with RA can help alleviate depressive symptoms.

We also observed significant decrements in HRQoL measures, specifically the MCS scores. This is not surprising, however, it is important given the strong association between patient-reported outcomes and disease activity ${ }^{93}$. It has also been suggested that patient-reported outcomes such as the HRQoL and other measures in clinical trials and routine clinical practice may shed light on variations in treatment response as well as the burden of disease among RA adults ${ }^{93,94}$. Our findings suggest that collecting patient-reported HRQoL can be critical in assessing disease burden that may not be captured by clinical assessment alone ${ }^{93}$.

Depression in working-age adults with RA was associated with substantial direct and indirect economic burden. For example, the presence of depression more than doubled the annual per person total healthcare costs, a number of missed work days and lost wages due to 
missed work days, even after controlling for predisposing factors, enabling characteristics, need factors, and personal health care practices. Although published evidence on the incremental economic impact of depression in RA is limited ${ }^{81}$, our findings are consistent with studies assessing the burden of depression on other chronic illness such as diabetes, cardiovascular disease and asthma have also reported the synergistic effect of depression in increasing the economic burden among individuals with chronic conditions ${ }^{76,95}$.

Our findings on the economic burden of depression in RA patients has important implications for the payers as it highlights an opportunity for reducing expenditures in RA patients by increasing efforts towards screening and effectively treating depression in RA patients. Potential strategies such as improving the integration of mental health services with rheumatology practice and facilitating mental health training for rheumatologists. Future studies need to explore whether treatment for depression provides an opportunity to reduce direct healthcare expenditures associated with depression in RA patients.

The study findings have important implications for the employers because depression costs U.S. employers more than $\$ 31$ billion annually due to missed work and decreased work performance ${ }^{96}$. One study done in the U.S. reported that depression leads to the highest reduction in work performance and the highest employer burden relative to any other chronic conditions ${ }^{97}$.Strategies that employers may adopt to improve mental health in employees include organizing workplace health promotion programs and stress management projects, which have shown the benefits of prevention and management of depression in workplace ${ }^{98,99}$.

To the best of our knowledge, this is the first population-based study that comprehensively examined the excess clinical, humanistic and economic burden of depression in working-age adults with RA. Other strengths of this study include the use of nationally 
representative survey, adjustment of a comprehensive list of confounders such as predisposing factors, enabling factors, need factors, and personal health care practices and the use of robust statistical techniques such as GLM, and recycled prediction in estimating the incremental costs and missed workdays.

However, the findings of this study should be interpreted considering its potential limitations. First, we did not control for the severity and duration of RA and depression as MEPS does not contain this information. These factors can be important confounders of both healthcare costs and work absence. Second, we have measured productivity loss as missed work days and did not consider other kinds of productivity loss such as reduced productivity while at work (presenteeism) and loss of employment.

\subsection{Conclusion}

Our findings would provide valuable insights to payers and other decision-makers to better understand the economic impact of comorbid depression on working RA patients from U.S. societal perspective. It is well-documented that depression in RA patients is often under-

recognized and under-treated in routine clinical practice ${ }^{32,100}$. Therefore, our study underscores the need for incorporating depression screening and management in the routine clinical management of RA in order to offset the substantial incremental costs associated with depression. Published evidence has well documented that depression is a treatable condition. However, it is still not clear whether depression treatment is equally effective in RA patients as compared to those with depression without RA ${ }^{101}$. Future studies need to assess the potential cost reductions that can be achieved through early detection and more aggressive treatment of depression in RA patients. 


\subsection{Acknowledgements and Data User Statement}

\section{Funding}

Research reported in this was supported by the NIGMS of the National Institutes of Health under award number U54GM104942. The content is solely the responsibility of the authors and does not necessarily represent the official views of the National Institutes of Health. 
Table 2.1. Description of Study Sample by Depression among Working-Age (18 - 64 years) Adults with Rheumatoid Arthritis

Medical Expenditure Panel Survey (2009, 2011, 2013 and 2015)

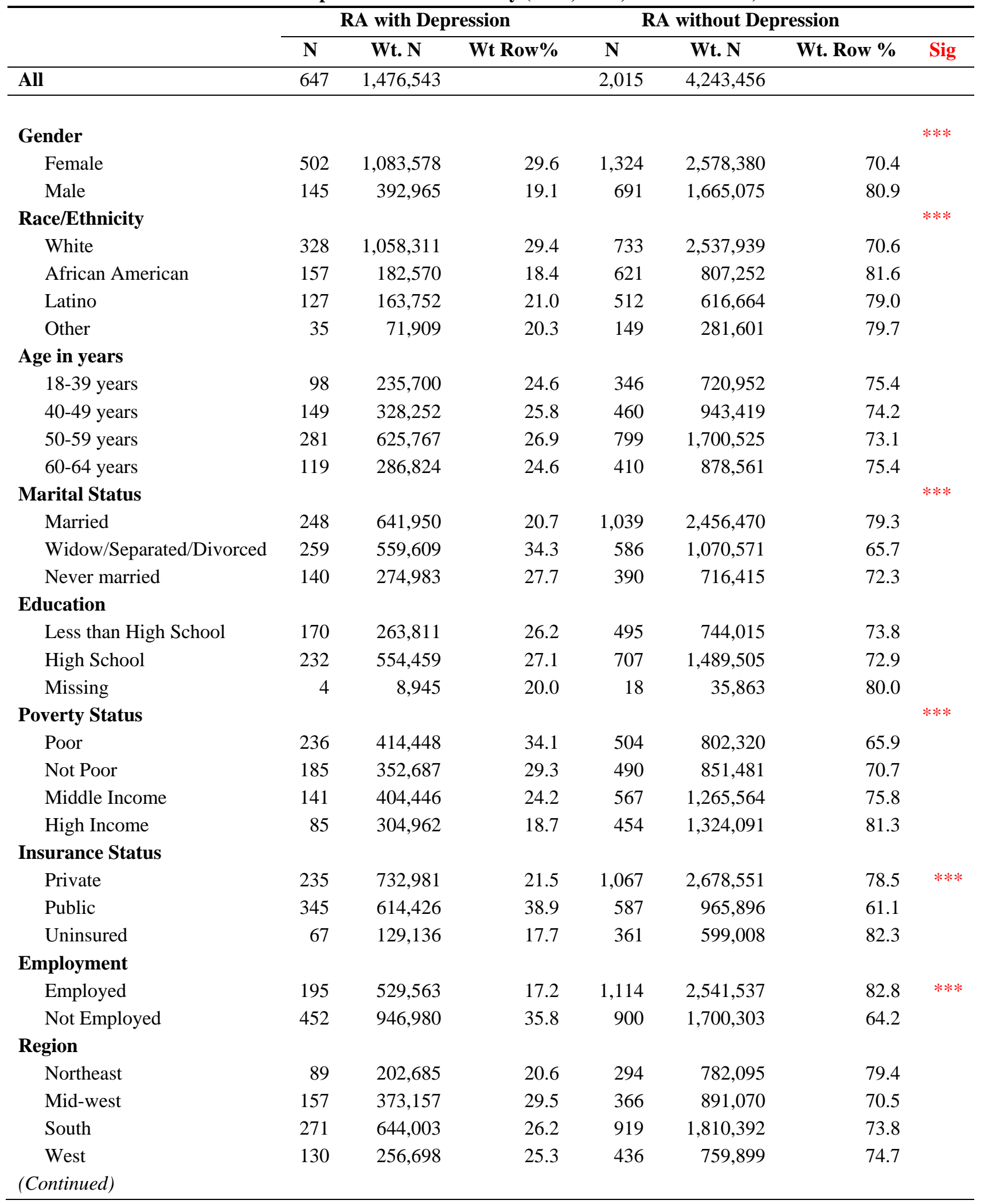


Table 2.1. Description of Study Sample by Depression among Working-Age (18 - 64 years) Adults with Rheumatoid Arthritis

Medical Expenditure Panel Survey (2009, 2011, 2013 and 2015)

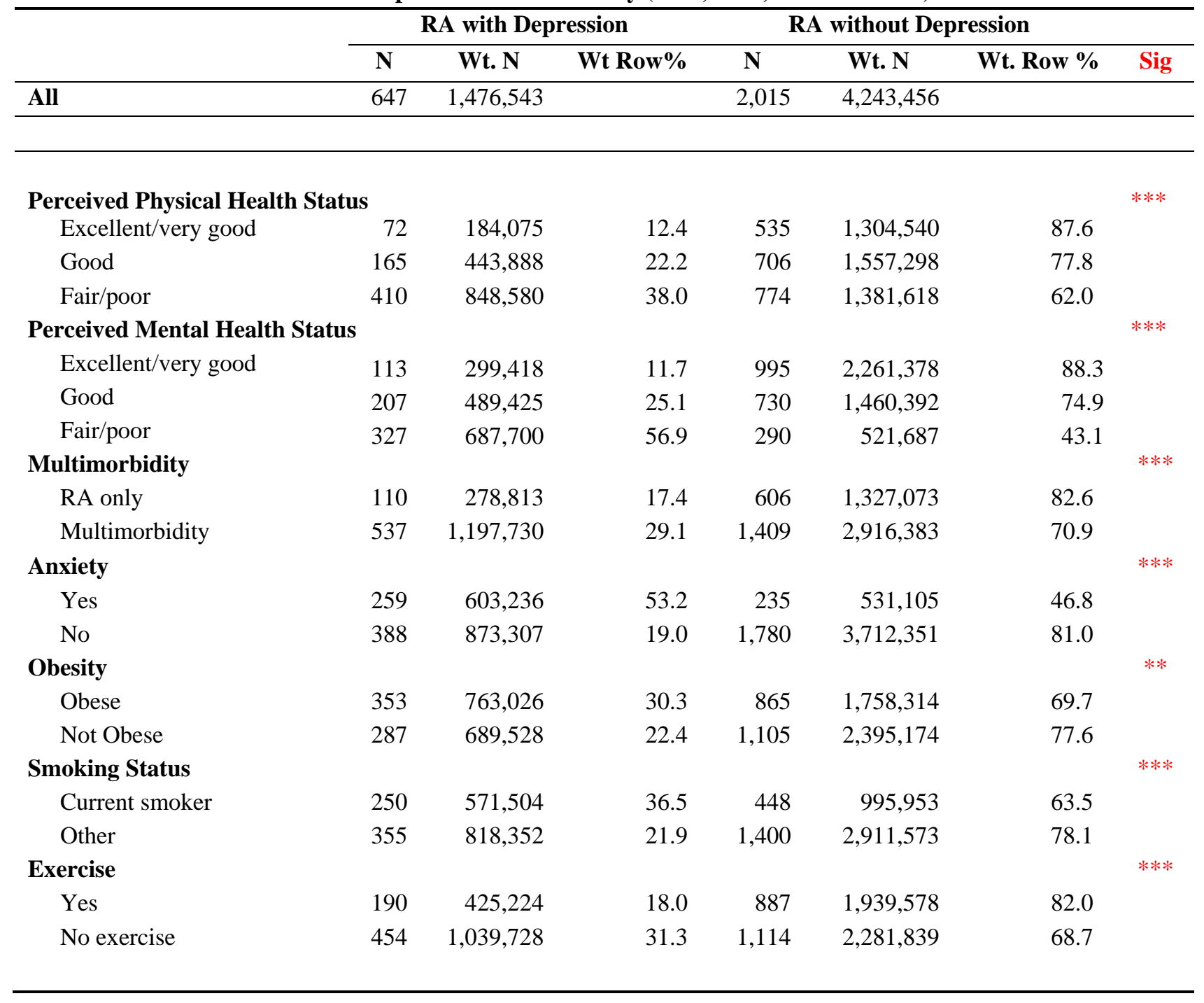

\section{Table 2.11}

\section{Table 2.12}

Note: Based on 2,662 adults with Rheumatoid Arthritis aged between 18 to 64 years, who were alive during the calendar year. Missing data for the following variables (education, obesity, smoking, and exercise) are not presented.

Asterisks represent significant group differences by the presence of depression based on chi-square tests. Weighted $\mathrm{N}$ and percentages were derived by dividing the person weights by the number of years pooled.

Wt. Weighted

$* * * \mathrm{p}<.001 ; * * .001 \leq \mathrm{p}<.01 ; * .01 \leq \mathrm{p}<.05$ 
Table 2.2. Clinical Outcome Associated with Depression among Working Age Adults with Rheumatoid Arthritis Medical Expenditure Panel Survey (2009, 2011, 2013 and 2015)

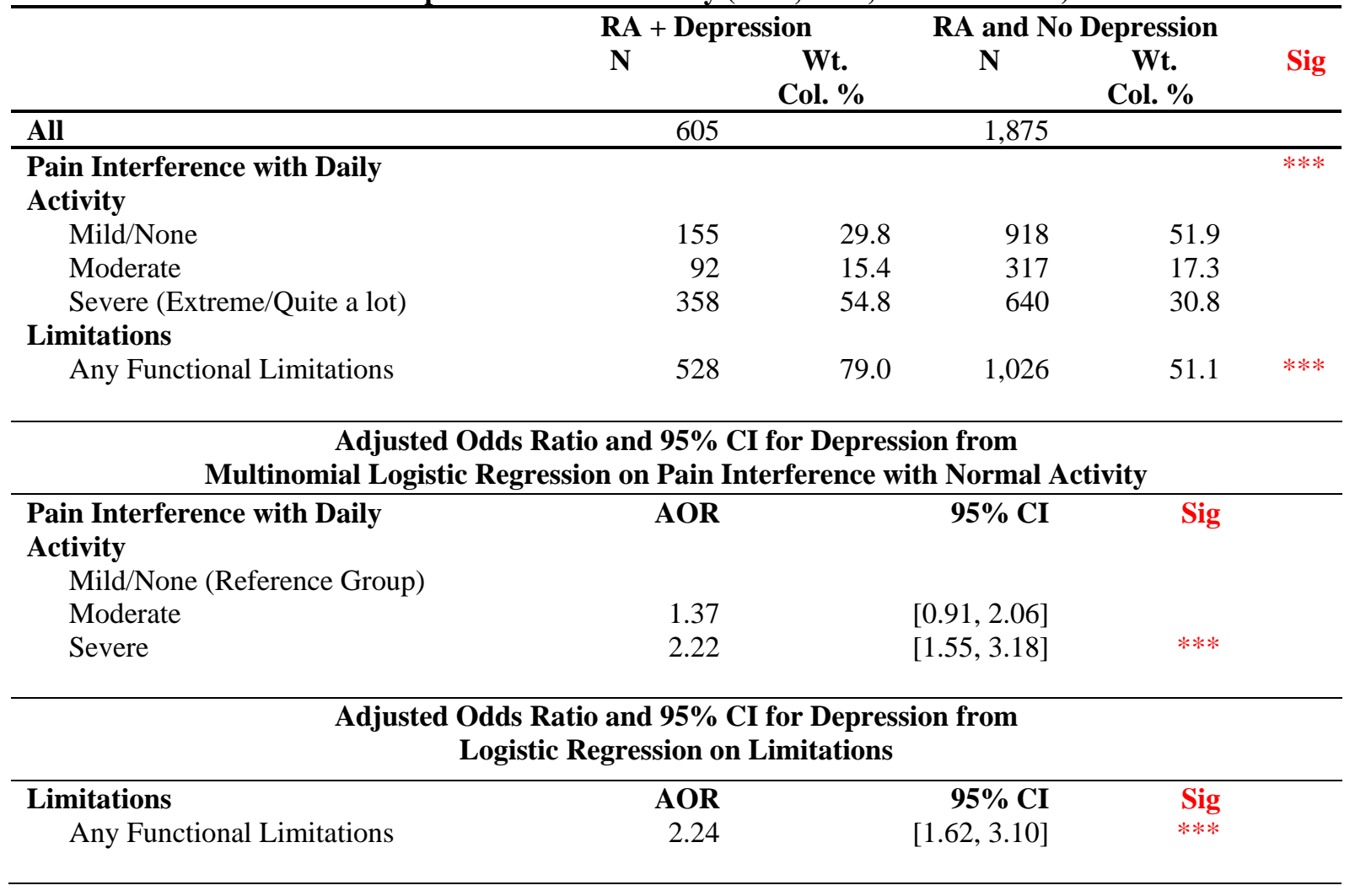

Note: Based on 2,662 adults with Rheumatoid Arthritis aged between 18 and 64 years, who were alive during the calendar year. Adjusted multinomial logistic regression controlled sex, race/ethnicity, age, region, marital status, education, family poverty status, health insurance, physical health status, mental health status, anxiety, multimorbidity, obesity, physical activity and smoking. Asterisks represent significant group differences by the presence of depression.

$* * * \mathrm{p}<.001 ; * * .001 \leq \mathrm{p}<.01 ; * .01 \leq \mathrm{p}<.05$.

Col: Column; Wt.: Weighted 
Table 2.3. Humanistic Outcomes (Health-Related Quality of Measures) by Presence of Depression among Working Age Adults with Rheumatoid Arthritis

Medical Expenditure Panel Survey (2009, 2011, 2013 and 2015)

\section{RA + Depression $\quad$ RA and No}

Depression

\begin{tabular}{|c|c|c|c|c|c|}
\hline & Wt. Mean & SE & Wt. Mean & SE & Sig \\
\hline All & $\mathrm{N}=647$ & & $\mathrm{~N}=2,015$ & & \\
\hline \multicolumn{6}{|l|}{$\begin{array}{l}\text { Physical Component Summary } \\
\text { Score }\end{array}$} \\
\hline \multicolumn{6}{|l|}{$\begin{array}{l}\text { Mental Component Summary } \\
\text { Score }\end{array}$} \\
\hline & 37.20 & 0.77 & 48.74 & 0.37 & $* * *$ \\
\hline \multicolumn{6}{|c|}{$\begin{array}{c}\text { Fully-Adjusted Model: } \begin{array}{c}\text { Parameter Estimates and Standard Errors for Depression } \\
\text { Ordinary Least Squares Regression } \\
\text { Mental Component Summary Score }\end{array}\end{array}$} \\
\hline & Beta & & Standard Error & Sig & \\
\hline $\begin{array}{l}\text { Depression } \\
\text { No Depression (Reference } \\
\text { Group) }\end{array}$ & -8.72 & & 0.81 & $* * *$ & \\
\hline \multicolumn{6}{|c|}{$\begin{array}{c}\text { Fully-Adjusted Model: Parameter Estimates and Standard Errors for Depression } \\
\text { Ordinary Least Squares Regression } \\
\text { Physical Component Summary Score }\end{array}$} \\
\hline & Beta & & Standard Error & Sig & \\
\hline $\begin{array}{l}\text { Depression } \\
\text { No Depression (Reference } \\
\text { Group) }\end{array}$ & -1.29 & & 0.81 & & \\
\hline
\end{tabular}

Note: Based on 2,662 adults with Rheumatoid Arthritis aged between 18 and 64 years, who were alive during the calendar year. Asterisks represent significant group differences by the presence of depression. The ordinary least squares regressions controlled for the following variables: sex, race/ethnicity, age, region, marital status, education, family poverty status, health insurance, anxiety, multimorbidity, obesity, physical activity and smoking.

SE: Standard error; Wt.: Weighted

$* * * \mathrm{p}<.001 ; * * .001 \leq \mathrm{p}<.01 ; * .01 \leq \mathrm{p}<.05$. 
Table 2.4. Economic Outcomes by Presence of Depression among Working Age Adults with Rheumatoid Arthritis

Medical Expenditure Panel Survey (2009, 2011, 2013 and 2015)

\begin{tabular}{|c|c|c|c|c|c|}
\hline & \multicolumn{2}{|c|}{ RA + Depression } & \multicolumn{2}{|c|}{ RA and No Depression } & \multirow[b]{2}{*}{ Sig } \\
\hline & Wt. Mean & $\mathbf{S E}$ & Wt. Mean & SE & \\
\hline All & $\mathrm{N}=647$ & & $\mathrm{~N}=2,015$ & & \\
\hline $\begin{array}{l}\text { Total Healthcare Expenditures } \\
(2015 \$)\end{array}$ & 17,941 & 1489 & 10,064 & 574 & **** \\
\hline $\begin{array}{l}\text { Total Out-of-Pocket Spending by } \\
\text { Patients/Families ( } 2015 \$)\end{array}$ & $\$ 1,443$ & 135 & $\$ 1,052$ & 73 & $* * *$ \\
\hline
\end{tabular}

Adjusted Total Direct Healthcare Expenditures of Depression

from Generalized Linear Models with Gamma Distribution and Log link

RA + Depression

RA and No Depression

\begin{tabular}{|c|c|c|c|c|c|}
\hline & Wt. Mean & $95 \% \mathrm{CI}$ & Wt. Mean & $95 \% \mathrm{CI}$ & Sig \\
\hline $\begin{array}{l}\text { Total Healthcare Expenditures } \\
(2015 \text { \$) }\end{array}$ & 14,752 & $\begin{array}{c}(14,411- \\
15,125)\end{array}$ & 10,541 & $\begin{array}{r}(10,206- \\
10,806)\end{array}$ & **** \\
\hline $\begin{array}{l}\text { Total Out-of-Pocket Spending by } \\
\text { Patients/Families }(2015 \$)\end{array}$ & 1,232 & $(1,202-1265)$ & 979 & $\begin{array}{l}(955- \\
1,005)\end{array}$ & $* * *$ \\
\hline
\end{tabular}

Incremental Total Direct Healthcare Expenditures of Depression from Counterfactual Recycled Prediction

Wt. Mean $\quad 95 \%$ CI

$\begin{array}{lrrr}\text { Total Healthcare Expenditures } & 4,212 & (4,114,4318) & * * * \\ (2015 \text { \$) } & 253 & (247-260) & * * * \\ \begin{array}{l}\text { Total Out-of-Pocket Spending by } \\ \text { Patients/their families }(2015 \$)\end{array} & & & *\end{array}$

Patients/their families (2015 \$)

\begin{tabular}{|c|c|c|c|c|c|}
\hline \multicolumn{6}{|c|}{ High Out-of-pocket Spending Burden (> 10\% Income Spent on Healthcare) } \\
\hline & \multicolumn{2}{|c|}{ RA + Depression } & \multicolumn{2}{|c|}{ RA and No Depression } & \multirow[b]{2}{*}{ Sig } \\
\hline & $\mathbf{N}$ & Wt. Col \% & $\mathbf{N}$ & Wt. Col \% & \\
\hline $\begin{array}{l}\text { High Out-of-pocket Spending } \\
\text { Burden }\end{array}$ & 192 & 30.7 & 424 & 21.3 & $* * *$ \\
\hline \multicolumn{6}{|c|}{$\begin{array}{l}\text { Fully-Adjusted Model: Adjusted Odds Ratio (AOR) and 95\% Confidence Intervals (CI) of Depression } \\
\text { from Logistic Regression on High Out-of-Pocket Burden }\end{array}$} \\
\hline & AOR & $95 \% \mathrm{CI}$ & Sig & & \\
\hline $\begin{array}{l}\text { Depression } \\
\text { No Depression (Reference) }\end{array}$ & 1.34 & {$[1.01,1.79]$} & ** & & \\
\hline
\end{tabular}

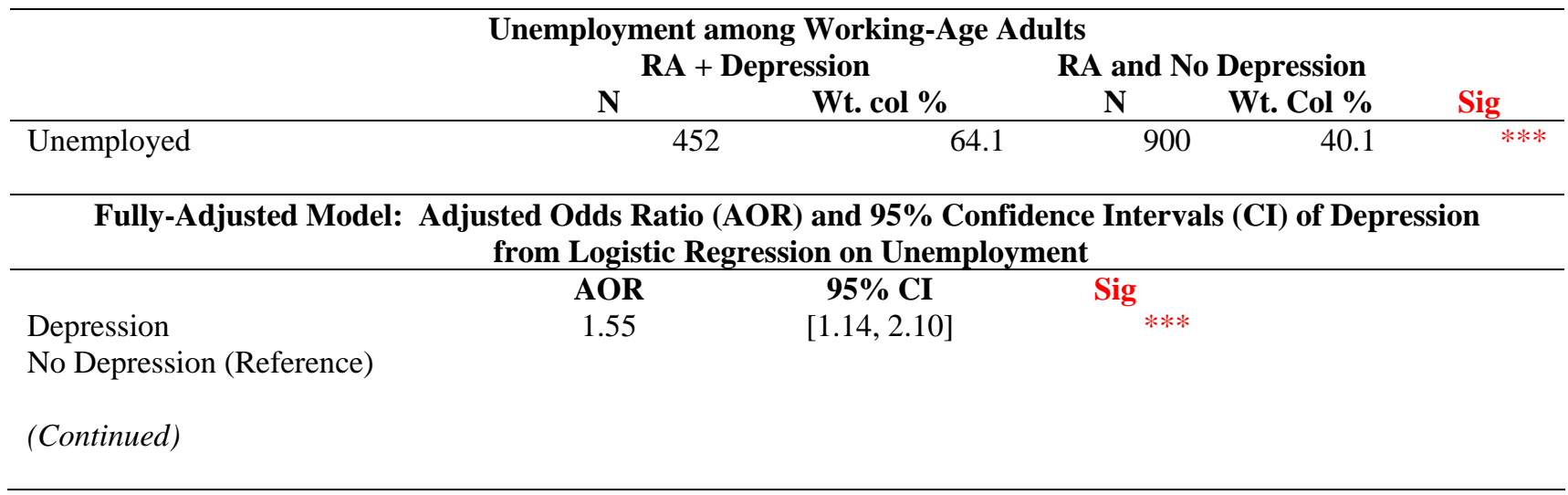


Table 2.4. Economic Outcomes by Presence of Depression among Working Age Adults with Rheumatoid Arthritis

Medical Expenditure Panel Survey (2009, 2011, 2013 and 2015)

Fully-Adjusted Models: Total Productivity Losses by Depression from from Negative Binomial Regression on Missed Work Days RA + Depression

RA and No Depression

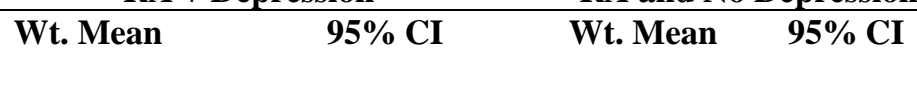

\begin{tabular}{llllll} 
Number of Missed Work Days & 9.0 & $(8.7-9.4)$ & 6.0 & $(5.7-6.2)$ & $* * *$ \\
Lost Wages & 853 & $(833-873)$ & 571 & $(558-584)$ & \\
\hline
\end{tabular}

Incremental Total Productivity Losses Associated with Depression from Counterfactual Recycled Prediction

$\begin{array}{lccc} & \text { Wt. Mean } & \mathbf{9 5 \%} \text { CI } & * \\ \text { Number of Missed Work Days } & 3.1 & (2.9-3.2) & * \\ \text { Lost Wages } & 282 & (276-289 & *\end{array}$

Note: Based on 2,662 adults with Rheumatoid Arthritis aged between 18 and 64 years, who were alive during the calendar year. Asterisks represent significant group differences by the presence of depression. The adjusted models controlled for the following variables: sex, race/ethnicity, age, region, marital status, education, family poverty status, health insurance, physical health, mental health status, anxiety, multimorbidity, obesity, physical activity and smoking.

Missed work days and lost wages were estimated only for those who were employed.

$* * * \mathrm{p}<.001 ; * * .001 \leq \mathrm{p}<.01 ; * .01 \leq \mathrm{p}<.05$. 


\section{CHAPTER 3}

\section{Depression and Treatment Response to Tumor Necrosis Factor Inhibitor Therapy}

among Working-Age Adults with Rheumatoid Arthritis: A Post Regression Non-Linear

\section{Decomposition Approach}

\subsection{Abstract}

Objective. We examined the relationship between prevalent depression and treatment response to Tumor Necrosis Factor Inhibitor(TNFi) therapy among working-age adults with Rheumatoid Arthritis(RA). Method. We adopted a retrospective cohort study design with data from a commercially-insured population using QuintilesIMS RWD Adjudicated Claims for the period October 1, 2009 to September 30, 2015. The study population $(\mathrm{N}=6,970)$ comprised workingage (18-63 years) adults with RA, who were initiated on TNFi therapy. Response to TNFi therapy during the 1-year follow-up period was measured using a validated claims-based algorithm. Logistic regression was used to examine the association between depression and treatment response to TNFi after adjusting for patient characteristics. A post-regression decomposition method was used to quantify how much of the difference in rates of treatment response reflected the explained and unexplained factors. Results. Overall, $12.5 \%$ of RA patients had depression. A significantly higher proportion of RA patients without depression responded to TNFi-therapy as compared to RA patients with depression (39.4\% vs. 31.5\%). After adjusting for baseline risk factors, adults with depression were $22 \%$ less likely to respond to TNFi therapy $(\mathrm{AOR}=0.78,95 \% \mathrm{CI}: 0.66,0.92)$. Decomposition analysis revealed that $36.7 \%$ of the difference in treatment response between the "depression" and "no depression group" were due to observed differences in patient characteristics such as the presence of anxiety, glucocorticoid use, and opioid use. Conclusion. These findings highlight the negative impact of depression on treatment 
response to TNFi and provide insights into specific subgroups of RA patients with depression who are at risk of responding poorly to TNFi.

\subsection{Introduction}

Tumor Necrosis Factor Inhibitors (TNFi) are the most widely used first-line biologic therapies in Rheumatoid Arthritis (RA) patients with moderate-to-severe disease who fail treatment with conventional DMARDS such as methotrexate, sulfasalazine and hydroxychloroquine ${ }^{57}$. Although TNFi therapy has proven to be highly efficacious in controlling disease activity in moderate-to-severe RA; there is considerable heterogeneity in treatment response to TNFi. In routine clinical practice, only one-third of RA patients respond to TNFi ${ }^{102}$. The variability in treatment response to TNFi may be associated with the presence of co-existing chronic conditions ${ }^{103}$. Depression is one of the highly prevalent chronic condition in RA patients 15 and may affect RA through shared biologic pathways ${ }^{100,104}$. Depression is now increasingly viewed as an inflammatory condition that is caused by an overactive immune system and may exert a negative influence on treatment response in RA by its effect on pro-inflammatory cytokines ${ }^{12,105}$. Therefore, it is important to understand the relation between depression and particular anti-cytokine therapies in RA such as TNFi.

Previous studies have shown a strong association between depression and disease activity in RA ${ }^{28,106,107}$. In a randomized controlled trial, researchers found that RA patients with baseline depression were more likely to have higher RA disease activity and physical disability as compared to those without depression ${ }^{28}$. This study also demonstrated those with depression were $50 \%$ less likely to respond to glucocorticoid treatment in RA compared to those without depression ${ }^{28}$. In another study of 59 RA patients treated in a Rheumatology Clinic in United Kingdom, RA patients with persistent depressive symptoms were found to have lower reductions 
in RA disease activity as compared to those without depression ${ }^{32}$. However, this was a descriptive study and the small sample size did not allow to adjust for other potential risk factors such as the demographics and the clinical characteristics of the patients. Another study done using the United States' RA registry data reported that the presence of depressive symptoms was associated with a reduced likelihood of clinical remission at 6 months but did not affect clinical remission significantly at 12 months ${ }^{29}$. However, this study assessed depression as one of the independent variables within a multivariate framework and did not evaluate the extent to which each of the individual patient level characteristics such as demographics and clinical factors, contributes to the difference in treatment response to TNFi by depression status.

The current study extends the literature by using post-regression non-linear decomposition technique to explain patient characteristics that may contribute to poor treatment response to TNFi among RA patients with depression. Such analysis can inform patients, payers and providers to identify patient subgroups that are at risk of responding poorly to TNFi and therefore target these patients for closer monitoring.

Therefore, the primary objective of this study was to examine the effect of coexisting depression on treatment response to TNFi therapy among working-age RA patients and examine the extent to which differences in individual patient characteristics explain the observed difference in treatment response between RA patients with depression and those without depression by using post-regression non-linear decomposition technique. We focused on working-age adults because the prevalence of depression is particularly high among working-age RA patients ${ }^{15,51}$. Lack of treatment response due to depression may further add to the disability burden and productivity losses among working-age RA patients ${ }^{108}$.

\section{Conceptual Framework}


The conceptual framework for this study is adapted from the Andersen's Expanded Behavioral Model ${ }^{69}$ which posits that the healthcare utilization by an individual is a function of predisposing factors (e.g. age, sex ), enabling factors (e.g. insurance plan type, calendar year of TNFi initiation), need factors (e.g. number of chronic physical conditions, anxiety, substance abuse, any emergency room visit, any inpatient visit, RA-related medication use) and external environment factors (e.g. geographical region). As the treatment response to TNFi was operationalized based on prescription drug utilization, Andersen's Behavioral Model was an appropriate conceptual model for selecting the independent variables in our study. The predisposing factors represent the individual characteristics that predict the propensity for the use of healthcare services. The enabling factors represent the factors that enable an individual to gain access to healthcare services. The need factors are those characteristics that define an individual's health status. The external environment represents the environmental and geographical factors that influence an individual's health service utilization.

\subsection{Methods}

\section{Study Design}

A retrospective observational cohort study design with baseline and follow-up period was used. The first observed prescription date for a TNFi biologic (adalimumab, infliximab, etanercept, golimumab and certolizumab pegol) between October 2010 and September 2014 was used as the index date. Using this index date, the study period was divided into 12 months preindex (i.e. baseline period) and 12 months post-index (i.e. follow up period).

\section{Data}

We used data from QuintilesIMS Real World Data Adjudicated Claims for the period October 1, 2009 to September 30, 2015. This is an integrated claims database that includes 
enrollment, medical and pharmacy claims information for more than 95 million enrollees of commercial plans across the US. The data includes the records of $90 \%$ of hospitals, $80 \%$ of doctors and $85 \%$ of large companies in the US. This database also records enrollees demographics (year of birth, gender, geographic region), plan type (health maintenance organization, preferred provider organization), payer type (commercial, self-insured), prescription drug information and considered nationally representative of commercially insured individuals less than 65 years of age in the US.

\section{Study Population}

The study population included adults aged between 18 and 63 years at the index date, with a diagnosis of RA (ICD-9-CM: 714.0x) in the baseline period and initiated on TNFi therapy between October 2010 and September 2014 (Supplemental Figure 7.1.1). Continuous enrollment in both pharmacy and medical benefits was required for the 12 months baseline period and 12 months follow up period. Adults with any biologic use during the baseline period were excluded. In addition, adults diagnosed with other autoimmune conditions for which biologics are used (Crohn's disease, ulcerative colitis, ankylosing spondylitis, psoriasis, psoriatic arthritis, juvenile idiopathic arthritis) during the baseline or treatment or follow-up period were excluded. Furthermore, adults diagnosed with Non-Hodgkin's lymphoma, chronic lymphocytic leukemia or HIV/AIDS were also excluded.

\section{Measures}

\section{Dependent variable: Response to Treatment with TNFi (Yes/No)}

Treatment response in RA is typically measured using Disease Activity Soring in 28 joints (DAS-28) and is considered a gold standard approach to measuring treatment response. However, DAS-28 are not available in claims data. Therefore, Curtis et.al developed a claims- 
based algorithm for measuring the treatment response to biologics in RA by using variables that are readily available in claims data such as medication use, adherence, switching, and dose escalation ${ }^{109}$. This algorithm was initially developed by using the Veteran Health Administration (VHA) claims database linked with VHA registry data and validated by using DAS-28 scores from the laboratory results from the VHA registry data. A high positive predictive value of $87 \%$ was found when the performance of this algorithm was tested using commercial claims data and change in DAS28 ${ }^{110}$.

\section{Claims-based Algorithm Components:}

As per the algorithm, RA patients initiating TNFi therapy were considered to have an effective response to TNFi therapy if they statisfied all of the following 6 criteria:

(1) Adherence to index TNFi: Adherence to TNFi was calculated using proportion of days covered (PDC). The PDC was calculated by using the date of service and the days of supply for each fill of the index TNFi. Patients with a PDC of at least $80 \%$ were considered adherent. Patients taking infliximab, were considered adherent if they had at least 7 infusions in the 1 -year treatment period.

(2) No increase in TNFi dose compared to the first dose: Dose increases were measured based on the type of TNFi medications. For Adalimumab (subcutaneous) users, the dose escalation criteria required no more than $40 \mathrm{mg}$ per week; for Certolizumab pegol (subcutaneous) users the dose escalation criteria was $\geq 200 \mathrm{mg}$ (after 56 days) of the index-dose; for Etanercept (subcutaneous) users, the dose escalation criteria was $\geq 100 \mathrm{mg} / \mathrm{week}$ of the index dose; for Golimumab (intravenous) users, the dose escalation criteria was $\geq 50 \mathrm{mg}$ from the first to the last dose; for Golimumab (subcutaneous) users the dose escalation criteria was $\geq 25$ $\mathrm{mg} /$ week post-index (i.e., $>50 \mathrm{mg}$ monthly post-index); for Infliximab users, the dose escalation 
criteria were $\geq 100 \mathrm{mg}$ from the first to last dose or $<11$ infusions during the 1 year post-index period or $\geq 7$ weeks between doses (after the third dose).

(3) No new conventional synthetic DMARD: No addition of methotrexate, sulfasalazine, leflunomide, or hydroxychloroquine in the treatment period in RA patients who did not have any claims for that drug in the baseline period.

(4) No switch to another biologic drug approved for use in patients with RA.

(5) No new/increased oral glucocorticoid: Patients with no claims for oral glucocorticoids in the baseline period: could not receive more than 30 days of oral glucocorticoids between the (index date +91 days) to (index date +365 days); for patients with claims of oral glucocorticoids, there should have been no increase in the oral glucocorticoid dose from the $6^{\text {th }}$ to the $12^{\text {th }}$ month after the index date compared to the 6 months before the index date. Escalation in oral glucocorticoid dose was determined based on prednisone equivalent dose for all glucocorticoids.

(6) At most one parenteral or intra-articular glucocorticoid joint injection on unique days in months 4 to 12 of the post-index period.

Using the above-mentioned components, we classified treatment response to TNFi as a dichotomous variable (Yes/No) during a one-year treatment period (one-year after the index date).

\section{Key Independent Variable: Prevalent Depression}

Depression in the baseline period was identified using a standard algorithm ${ }^{111,112}$. Adults with ICD-9-CM codes of 296.2 (major depressive disorder, single episode), 296.3 (major depressive disorder, recurrent episode), 298.0 (depressive type psychosis), 300.4 (neurotic 
depression), 309.1 (prolonged depressive reaction), and 311 (depressive disorder, not classified) during the baseline period were considered to have co-existing depression.

\section{Other independent variables:}

Other independent variables measured during the baseline period included predisposing characteristics - age, sex, enabling characteristics insurance plan type(health maintenance organizations [HMOs]; preferred provider organizations [PPOs]), need characteristics included the total number of chronic physical conditions, anxiety and substance abuse, healthcare utilization (any inpatient and any emergency room visit), and RA related medication use (methotrexate, other DMARDs, glucocorticoids, NSAIDs and opioids). The total number of chronic physical conditions was measured using the Clinical Classifications Software managed by the Agency for Healthcare Research and Quality (AHRQ). The external environment characteristics included the index year of TNFi initiation and geographic region (East, Midwest, South, and West).

\section{Statistical Analysis}

We used chi-square tests to examine the unadjusted differences in treatment response by the presence of depression. To examine the association between depression and treatment response to TNFi after adjusting for the pre-disposing, enabling, need and external environment factors, adjusted logistic regressions were conducted separately for RA patients with depression and RA patients without depression and the two groups combined (RA patients with and without depression). A post-regression non-linear decomposition technique was used to examine the extent to which individual patient characteristics explained the difference in treatment response between the group with depression and the group without depression. 
Previous research has often used a binary indicator variable for depression within a multivariate framework and did not examine how much of the observed difference in treatment response can be explained by differences in characteristics between the depression and no depression group. The decomposition method aids in identifying which patient-level factors accounted for how much of the difference in the treatment response between the group with depression and the group without depression. The decomposition technique is a sophisticated econometric method that quantifies how much of the difference in rates reflects the observable or explained factors (e.g. sex, age, medications) versus unobservable or unexplained factors (e.g. physiologic difference, lifestyle difference, education, income). In this study, as the dependent variable was binary (depression vs. no depression), we used the nonlinear decomposition technique proposed by Fairlie ${ }^{113}$ and modified by Yun ${ }^{114}$. We conducted logistic regression analyses using Statistical Analysis Software (SAS) version 9.3, Cary, NC, USA and decomposition with STATA 14.

\subsection{Results}

Table 3.1 presents the characteristics of the study population by the presence of depression in the baseline period. Of the 6,970 working-age patients with RA, initiated on TNFi therapy, an overwhelming majority were female $(75.9 \%)$ and the mean age was 50 years. Overall, $12.5 \%$ of the study population had co-existing depression during the baseline period. More than one-third of the study population had at least one additional chronic physical condition.

There were statistically significant differences in the baseline characteristics of those with depression versus those without depression with respect to sex, the number of chronic physical conditions, any emergency room visit, any inpatient visit, substance abuse, anxiety and RA 
related medication use (Table 3.1). For example, a significantly higher proportion of RA patients with depression were female ( $88.2 \%$ vs. $74.2 \%$ ), had comorbid anxiety ( $28 \%$ vs. $6 \%$ ), had 1 to 3 additional chronic physical conditions (41.6\% vs. 32.6\%), had an emergency room visit during the baseline period (36.9\% vs. $21.6 \%$ ), and had RA-related medication use compared to those without depression.

\section{Response to TNFi treatment by Depression:}

Based on the algorithm, 39.8\% $(\mathrm{N}=2,676)$ of the study population responded to TNFi therapy during the one-year follow-up period (Table 3.2). The most common reason for nonresponse to TNFi therapy was low adherence to index TNFi (43.1\%). A significantly lower proportion of RA patients with co-existing depression responded to treatment with TNFi therapy as compared to RA patients without co-existing depression (31.5\% vs. 39.4\%; $<$.001) (Table $3.2)$.

The top panel of Table 3.3 displays results from the pooled logistic regression of treatment response to TNFi. In this regression, RA patients with depression and without depression were combined and the difference in treatment response between those with depression vs. those without depression was measured using an indicator variable for depression (with no depression as the reference group), after adjusting for the explanatory variables. After adjusting for baseline risk factors, those with depression were $22 \%$ less likely to respond to TNFi therapy compared to those without depression $(\mathrm{AOR}=0.78,95 \% \mathrm{CI}: 0.66,0.92)$.

Table 3 also presents AORs and 95\% CIs from the logistic regression on treatment response to TNFi conducted separately for RA patients with depression and without depression. We observed differences in associations between the independent variables and treatment response by the depression status. For example, among those without depression, females were 
significantly less likely to respond to TNFi treatment as compared to males ( $\mathrm{AOR}=0.88 ; 95 \%$ $\mathrm{CI}=0.78,0.99)$; there was no sex difference in treatment response among adults with depression. Among RA patients without depression, those who had anxiety were significantly less likely to respond to TNFi treatment, whereas among RA patients with depression there were no differences in treatment response by presence of anxiety.

The results from the decomposition of the differences between RA patients with depression vs. RA patients without depression in treatment response to TNFi is shown in Table 3.4. Overall, there was a 7.9 percentage point difference in treatment response to TNFi between RA patients with depression vs. RA patients without depression $(\mathrm{p}<0.001)$. The differences in observed characteristics between RA patients with depression and those without depression explained only $37.1 \%$ (i.e. 2.9 percentage points out of the 7.9 percentage point difference). This implies that the difference in treatment response to TNFi between those with depression versus those without depression would be reduced by $37.1 \%$ if those with depression had a similar distribution of baseline characteristics as those without depression.

Table 3.4 also summarized the individual contribution of factors to the disparity in treatment response rate between depression and no depression group. A positive sign represents a reduction in the disparity in treatment response between depression and no depression group and a negative sign represents an increase in disparity in treatment response between the depression and no depression group. Presence of anxiety in the baseline was a major contributor to the total difference in treatment response to TNFi and accounted for $19.2 \%$ of the explained portion in treatment response. If all baseline characteristics remained the same and if the prevalence pattern for anxiety was the same between RA patients with and without anxiety, the difference in treatment response would drop by 1.5 percentage points to 6.4 percentage points. 
The use of RA-related medications followed different patterns. For example, glucocorticoids and opioid pain medication explained $17.9 \%$ of the total difference in treatment response rate. If RA patients with depression had the same rate of glucocorticoids and opioid pain medication use, then the difference in treatment response between depression and no depression group would be reduced by 1.4 percentage points (i.e. from $7.9 \%$ to $6.5 \%$ ). However, if RA patients with depression had the same rates of NSAIDs, methotrexate, and other DMARDs, then the gap in treatment response rate between the depression and no depression will actually increase by 0.74 percentage points (i.e. from $7.9 \%$ to 8.64 ).

\subsection{Discussion}

In this study, we used large administrative claims data to examine the rate of treatment response to TNFi for RA patients with depression in comparison to RA patients without depression and quantified how much of the difference in treatment response rates between the two groups reflected differences in observable characteristics between the two groups. We found that working-age adults with RA and depression were less likely to respond to treatment compared to those without depression. A plausible explanation is that depression may affect treatment response to anti-rheumatic therapies through dysregulation of inflammatory pathways ${ }^{115}$. This is consistent with studies that have documented the negative effect of depression on disease activity and clinical remission in RA $28,29,106,107$. It is also possible that adults with depression may not adhere to therapies, which may affect treatment response. Many studies have documented the negative effects of depression on treatment adherence in RA patients ${ }^{48,116}$. In our study, we observed that a significantly higher percentage of RA patients with depression had low adherence to TNFi compared to those without depression (49.8\% vs 
42.2\%). Such non-adherence to treatments associated with depression may further heighten the disability burden and productivity loss among working-age RA patients (Li, Gignac et al. 2006).

Our findings also underscore the need for co-managing RA and depression. There is preliminary evidence that depression treatment can improve treatment response in patients with arthritis. Emerging research suggests that antidepressants used for treating depression can improve RA treatment efficacy ${ }^{117}$. A recent case report suggested that specific Serotonin reuptake inhibitor antidepressant drugs, often used to treat depression, may improve clinical outcomes in RA patients ${ }^{118}$. Furthermore, the anti-inflammatory properties of antidepressants may help alleviate pain and fatigue in rheumatoid arthritis ${ }^{119}$. If more studies confirm the association between depression treatment and treatment response to TNFi therapy, then depression treatment needs to be integrated into RA care to improve clinical outcomes.

Results from the post-regression decomposition analyses revealed that differences in anxiety prevalence explained a substantial portion of the observed difference in treatment response to TNFi. It is well-documented that anxiety disorders are common in patients with major depression ${ }^{120,121}$. Co-occurring anxiety and depression in RA patients may with associated with higher symptom severity and disease activity leading to sub-optimal treatment response ${ }^{122}$. Therefore, our findings suggest that RA patients with co-existing anxiety and depression should be monitored more closely and should be treated with effective pharmacological and behavioral therapies to improve treatment response in RA.

In addition to anxiety, differences in baseline RA-related medication use among RA patients with depression and no depression also contributed to the observed difference in treatment response to TNFi. For example, RA patients with depression were more likely to use glucocorticoids and opioid pain medications as compared to RA patients without depression. Our 
findings suggest that reducing the rate of glucocorticoid and opioid medication use in RA patients with depression would significantly reduce the gap in treatment response to TNFi. This is because the long-term use of both glucocorticoids and opioid medications have been associated with increased risk of depression ${ }^{123,124}$.

We also found that only $37.1 \%$ of the differences in response rate between depression and no depression group can be explained by observed differences in patient characteristics. The explained portion could have been low because our study did not include life-style risk factors, the severity of illness, and other physiologic that may explain treatment response.

The findings of this study need to be interpreted in the context of its limitations and strengths. The strengths include a large commercially insured population that is nationally representative of working-age adults, ability to measure treatment effectiveness, use of decomposition methods, and measurement of concomitant medications. Potential limitations of the study include the lack of severity measures for RA and depression. However, by including only those who were initiated on TNFi, we ensured that the patients in our study had moderate or severe RA. Similarly, by using major depressive disorder, we included only those with clinically significant depression. It is possible that depression was underdiagnosed and the "no depression" group included those with depression as well. This may have underestimated the differences in treatment response. We did not have information on individual characteristics such as race, body mass index, exercise and smoking status that may affect treatment response and underestimate the contribution of explanatory variables to the disparity in treatment response between the depression and no depression group. In claims data, we can only observe filled prescriptions of medications and not actual use of medication. Although we used a "wash out" 
period to identify TNFi initiators, we cannot assert they are truly naïve to TNF inhibitors because they may have used it before the washout period (i.e. 12 months).

\subsection{Conclusion}

Notwithstanding the limitations, our study provides important insights on the negative effect of comorbid depression on treatment response to TNFi from a population-based perspective. Future studies need to assess whether increasing diagnosis and treatment of depression in RA patients may improve treatment response and clinical outcome associated with particular anti-rheumatic therapies.

\subsection{Acknowledgements and Data User Statement}

\section{Funding}

Research reported in this was supported by the NIGMS of the National Institutes of Health under award number U54GM104942. The content is solely the responsibility of the authors and does not necessarily represent the official views of the National Institutes of Health.

\section{Data User Statement}

The statements, findings, conclusions, views, and opinions contained and expressed in this article are based in part on data obtained under license from the following QuintilesIMS information services: QuintilesIMS Real-World Data Adjudicated Claims - US, October 2009September 2015, QuintilesIMS Health Incorporated. All Rights Reserved. The statements, findings, conclusions, views, and opinions contained and expressed herein are not necessarily those of QuintilesIMS Health Incorporated or any of its affiliated or subsidiary entities. 
Table 3.1. Description of Working-age Adults with Rheumatoid Arthritis initiated on TNFi by Depression Status During Baseline

QuintilesIMS Real-World Data Adjudicated Claims Database, 2009 - 2015

\begin{tabular}{|c|c|c|c|c|c|c|c|}
\hline & \multicolumn{2}{|c|}{ Total } & \multicolumn{2}{|c|}{ Depression } & \multicolumn{2}{|c|}{ No Depression } & \multirow[b]{2}{*}{ Sig } \\
\hline & $\mathbf{N}$ & $\%$ & $\mathbf{N}$ & $\%$ & $\mathbf{N}$ & $\%$ & \\
\hline All & 6,970 & 100.0 & 868 & 100 & 6,102 & 100 & \\
\hline \multicolumn{8}{|c|}{ Predisposing Characteristics } \\
\hline Sex & & & & & & & $* * *$ \\
\hline Female & 5,293 & 75.9 & 766 & 88.2 & 4,527 & 74.2 & \\
\hline Male & 1,677 & 24.1 & 102 & 11.8 & 1,575 & 25.8 & \\
\hline \multicolumn{8}{|l|}{ Age } \\
\hline 18-34 years & 612 & 8.8 & 90 & 10.4 & 522 & 8.6 & \\
\hline $35-44$ years & 1,155 & 16.6 & 154 & 17.7 & 1,001 & 16.4 & \\
\hline $45-54$ years & 2,500 & 35.9 & 311 & 35.8 & 2,189 & 35.9 & \\
\hline $55-63$ years & 2,703 & 38.8 & 313 & 36.1 & 2,390 & 39.2 & \\
\hline \multicolumn{8}{|c|}{ Enabling Characteristics } \\
\hline \multicolumn{8}{|c|}{ Insurance Plan Type } \\
\hline HMO & 691 & 9.9 & 88 & 10.1 & 603 & 9.9 & \\
\hline PPO & 5,416 & 77.7 & 664 & 76.5 & 4,752 & 77.9 & \\
\hline Other & 863 & 12.4 & 116 & 13.4 & 747 & 12.2 & \\
\hline \multicolumn{8}{|c|}{ Need Characteristics } \\
\hline Number of Chroni & sical Con & & & & & & **** \\
\hline 0 & 4,428 & 63.5 & 467 & 53.8 & 3,961 & 64.9 & \\
\hline 1 to 3 & 2,351 & 33.7 & 361 & 41.6 & 1,990 & 32.6 & \\
\hline$>3$ & 191 & 2.7 & 40 & 4.6 & 151 & 2.5 & \\
\hline Substance Abuse & & & & & & & $* * *$ \\
\hline Yes & 1,638 & 23.5 & 86 & 9.9 & 373 & 6.1 & \\
\hline No & 5,332 & 76.5 & 782 & 90.1 & 5,729 & 93.9 & \\
\hline Anxiety & & & & & & & $* * *$ \\
\hline Yes & 614 & 8.8 & 243 & 28.0 & 365 & 6.0 & \\
\hline No & 6,356 & 91.2 & 625 & 72.0 & 5,737 & 94.0 & \\
\hline \multicolumn{8}{|c|}{ Health Services Utilization } \\
\hline ED Visits & & & & & & & **** \\
\hline Yes & 459 & 6.6 & 320 & 36.9 & 1,318 & 21.6 & \\
\hline No & 6,511 & 93.4 & 548 & 63.1 & 4,784 & 78.4 & \\
\hline IP Visits & & & & & & & $* * *$ \\
\hline Yes & 608 & 8.7 & 149 & 17.2 & 465 & 7.6 & \\
\hline No & 6,362 & 91.3 & 719 & 82.8 & 5,637 & 92.4 & \\
\hline \multicolumn{8}{|c|}{ Healthcare Utilization - Medication Use } \\
\hline NSAIDS & & & & & & & *** \\
\hline Yes & 3,883 & 55.7 & 524 & 60.4 & 3,359 & 55 & \\
\hline No & 3,087 & 44.3 & 344 & 39.6 & 2,743 & 45 & \\
\hline Methotrexate & & & & & & & * \\
\hline Yes & 5,080 & 72.9 & 659 & 75.9 & 4,421 & 72.5 & \\
\hline No & 1,890 & 27.1 & 209 & 24.1 & 1,681 & 27.5 & \\
\hline Other DMARDS $\uparrow$ & & & & & & & $* * *$ \\
\hline Yes & 3,491 & 50.1 & 492 & 56.7 & 2,999 & 49.1 & \\
\hline No & 3,479 & 49.9 & 376 & 43.3 & 3,103 & 50.9 & \\
\hline Glucocorticoids & & & & & & & $* *$ \\
\hline Yes & 5,025 & 72.1 & 663 & 76.4 & 4,362 & 71.5 & \\
\hline No & 1,945 & 27.9 & 205 & 23.6 & 1,740 & 28.5 & \\
\hline
\end{tabular}


Table 3.1. Description of Working-age Adults with Rheumatoid Arthritis initiated on TNFi by Depression Status During Baseline

QuintilesIMS Real-World Data Adjudicated Claims Database, 2009 - 2015

\begin{tabular}{|c|c|c|c|c|c|c|c|}
\hline & \multicolumn{2}{|c|}{ Total } & \multicolumn{2}{|c|}{ Depression } & \multicolumn{2}{|c|}{ No Depression } & \multirow[b]{2}{*}{ Sig } \\
\hline & $\mathbf{N}$ & $\%$ & $\mathbf{N}$ & $\%$ & $\mathbf{N}$ & $\%$ & \\
\hline Opioids & & & & & & & **** \\
\hline Yes & 3,912 & 56.1 & 638 & 73.5 & 3,274 & 53.7 & \\
\hline No & 3,058 & 43.9 & 230 & 26.5 & 2,828 & 46.3 & \\
\hline \multicolumn{8}{|c|}{ External Environment Characteristics } \\
\hline \multicolumn{8}{|l|}{ Index Year } \\
\hline 2010 & 401 & 5.8 & 46 & 5.3 & 355 & 5.8 & \\
\hline 2011 & 1,813 & 26.0 & 221 & 25.5 & 1,592 & 26.1 & \\
\hline 2012 & 1,878 & 26.9 & 262 & 30.2 & 1,616 & 26.5 & \\
\hline 2013 & 1,713 & 24.6 & 218 & 25.1 & 1,495 & 24.5 & \\
\hline 2014 & 1,165 & 16.7 & 121 & 13.9 & 1,044 & 17.1 & \\
\hline \multicolumn{8}{|l|}{ Region } \\
\hline East & 1,213 & 17.4 & 152 & 17.5 & 1,061 & 17.4 & \\
\hline Midwest & 2,083 & 29.9 & 275 & 31.7 & 1,808 & 29.6 & \\
\hline South & 3,077 & 44.1 & 348 & 40.1 & 2,729 & 44.7 & \\
\hline West & 597 & 8.6 & 93 & 10.7 & 504 & 8.3 & \\
\hline
\end{tabular}

Note: Based on 6,970adults with Rheumatoid arthritis aged between 18 and 63 as of the index date, initiated on Tumor Necrosis Factor Inhibitors between 2010 and 2014, with continuous enrollment for 12 months baseline and 12 months follow-up periods in a commercial insurance plan.

Asterisks represent significant group differences by the presence of depression based on chi-square tests.

$\dagger$ include Sulfasalazine, Leflunomide, Hydroxychloroquine, Azathioprine, Chloroquine, Cyclophosphamide, Cyclosporine, Minocycline, and Sodium Aurothiomalate.

DMARDS: Disease modifying anti-rheumatic drugs; ED: Emergency department; HMO: Health maintenance organization; PPO: Preferred provider organization; IP: Inpatient; NSAIDS: Nonsteroidal anti-inflammatory drugs. $* * * \mathrm{p}<.001 ; * * .001 \leq \mathrm{p}<.01 ; * .01 \leq \mathrm{p}<.05$ 
Table 3.2. Components of the Algorithm for TNFi Response Status at 1 year after TNFi Initiation Working-age Adults with Rheumatoid Arthritis initiated on TNFi

By Depression Status

QuintilesIMS Real-World Data Adjudicated Claims Database, 2009 - 2015

Low adherence to index TNFi

ALL

Depression

No Sig

$(\mathrm{PDC}<0.8)$

$43.1 \%$

Switched to new biologics

$23.5 \%$

$49.8 \%$

Depression

Dose escalation of TNFi

$8.9 \%$

$27.4 \%$

$42.2 \%$

$22.9 \%$

$10.6 \%$

$8.6 \%$

Conventional DMARD addition after

index

$3.3 \%$

$2.1 \%$

$3.4 \%$

Dose increase of oral glucocorticoid

$15.8 \%$

$19.7 \%$

$15.2 \%$

$2.6 \%$

$4.6 \%$

$2.3 \%$

$31.5 \%$

$39.4 \%$

Responded to TNFi Therapy

$38.4 \%$

$68.5 \%$

$60.6 \%$

Note: Based on 6,970 adults with Rheumatoid Arthritis aged between 18 and 63 as of the index date, initiated on Tumor Necrosis Factor Inhibitors between 2010 and 2014, with continuous enrollment for 12 months baseline and 12 months follow-up periods in a commercial insurance plan.

Asterisks represent significant group differences by the presence of depression based on chi-square tests.

Individual components of TNFi response criteria will not add to $38.4 \%$ because an individual may satisfy one or more components of the TNFI response algorithm.

PDC: Proportion of days covered; TNFi: Tumor Necrosis Factor Inhibitor 
Table 3.3. Adjusted Odds Ratios (AOR) and 95\% Confidence intervals (CI) from Separate Logistic Regressions on Response to TNFi by Depression Status Working-age Adults with Rheumatoid Arthritis Initiated on TNFi QuintilesIMS Real-World Data Adjudicated Claims Database, 2009 - 2015

\begin{tabular}{|c|c|c|c|c|c|c|c|c|c|}
\hline & \multicolumn{3}{|c|}{ Depression } & \multicolumn{3}{|c|}{ No Depression } & \multicolumn{3}{|c|}{ Pooled Regression } \\
\hline & AOR & $95 \% \mathrm{CI}$ & Sig & AOR & $95 \% \mathrm{CI}$ & Sig & AOR & $95 \% \mathrm{CI}$ & Sig \\
\hline \multicolumn{10}{|l|}{ Depression } \\
\hline Yes & & & & & & & 0.78 & {$[0.66,0.92]$} & $* *$ \\
\hline No & & & & & & & Ref & & \\
\hline \multicolumn{10}{|c|}{ Predisposing Characteristics } \\
\hline \multicolumn{10}{|l|}{ Sex } \\
\hline Female & 1.01 & {$[0.64,1.60]$} & & 0.88 & {$[0.78,0.99]$} & $*$ & 0.88 & {$[0.79,0.99]$} & $*$ \\
\hline Male & Ref & & & & & & Ref & & \\
\hline \multicolumn{10}{|l|}{ Age } \\
\hline 18-34 years & Ref & & & Ref & & & Ref & & \\
\hline $35-44$ years & 1.21 & {$[0.65,2.23]$} & & 1.09 & {$[0.87,1.36]$} & & 1.10 & {$[0.89,1.36]$} & \\
\hline $45-54$ years & 1.39 & {$[0.79,2.43]$} & & 1.25 & {$[1.02,1.54]$} & $*$ & 1.27 & {$[1.05,1.54]$} & $*$ \\
\hline $55-63$ years & 1.89 & {$[1.07,3.34]$} & $*$ & 1.32 & {$[1.07,1.63]$} & $* *$ & 1.38 & {$[1.14,1.68]$} & $* *$ \\
\hline \multicolumn{10}{|c|}{ Enabling Characteristics } \\
\hline \multicolumn{10}{|c|}{ Insurance Plan Type } \\
\hline HMO & Ref & & & Ref & & & Ref & & \\
\hline PPO & 1.41 & {$[0.82,2.43]$} & & 1.01 & {$[0.84,1.22]$} & & 1.03 & {$[0.87,1.23]$} & \\
\hline Other & 1.95 & {$[1.03,3.70]$} & $*$ & 1.25 & {$[1.00,1.56]$} & & 1.30 & {$[1.05,1.60]$} & $*$ \\
\hline \multicolumn{10}{|c|}{ Need Characteristics } \\
\hline \multicolumn{10}{|c|}{ Number of Chronic Physical Conditions } \\
\hline 0 & Ref & & & Ref & & & Ref & & \\
\hline 1 to 3 & 0.89 & {$[0.65,1.23]$} & & 1.06 & {$[0.94,1.19]$} & & 1.04 & {$[0.93,1.16]$} & \\
\hline$>3$ & 0.56 & {$[0.25,1.27]$} & & 0.91 & {$[0.63,1.30]$} & & 0.85 & {$[0.61,1.18]$} & \\
\hline \multicolumn{10}{|c|}{ Substance Abuse } \\
\hline Yes & 1.28 & {$[0.72,2.29]$} & & 0.92 & {$[0.71,1.19]$} & & 0.96 & {$[0.76,1.21]$} & \\
\hline No & Ref & & & Ref & & & Ref & & \\
\hline \multicolumn{10}{|l|}{ Anxiety } \\
\hline Yes & 1.10 & {$[0.79,1.55]$} & & 0.72 & {$[0.57,0.92]$} & $* *$ & 0.83 & {$[0.69,1.01]$} & \\
\hline No & Ref & & & Ref & & & Ref & & \\
\hline
\end{tabular}
Health Services Utilization

\begin{tabular}{|c|c|c|c|c|c|c|c|c|c|}
\hline \multicolumn{10}{|l|}{ ED Visit } \\
\hline Yes & 0.70 & {$[0.50,0.99]$} & $*$ & 0.88 & {$[0.76,1.01]$} & & 0.85 & {$[0.75,0.96]$} & $*$ \\
\hline No & Ref & & & Ref & & & Ref & & \\
\hline \multicolumn{10}{|l|}{ IP Visit } \\
\hline Yes & 1.21 & {$[0.79,1.87]$} & & 1.06 & {$[0.86,1.32]$} & & 1.09 & {$[0.90,1.32]$} & \\
\hline No & Ref & & & Ref & & & Ref & & \\
\hline \multicolumn{10}{|c|}{ Health Services Utilization - Medication Use } \\
\hline \multicolumn{10}{|c|}{ NSAIDS } \\
\hline Yes & 0.97 & {$[0.71,1.32]$} & & 1.15 & {$[1.03,1.28]$} & * & 1.13 & {$[1.02,1.25]$} & $*$ \\
\hline No & $\operatorname{Ref}$ & & & Ref & & & Ref & & \\
\hline
\end{tabular}

(Continued) 
Table 3.3. Adjusted Odds Ratios (AOR) and 95\% Confidence intervals (CI) from Separate Logistic Regressions on Response to TNFi by Depression Status Working-age Adults with Rheumatoid Arthritis Initiated on TNFi QuintilesIMS Real-World Data Adjudicated Claims Database, 2009 - 2015

\begin{tabular}{|c|c|c|c|c|c|c|c|c|c|}
\hline & \multicolumn{3}{|c|}{ Depression } & \multicolumn{3}{|c|}{ No Depression } & \multicolumn{3}{|c|}{ Pooled Regression } \\
\hline & AOR & $95 \% \mathrm{CI}$ & Sig & $\overline{A O R}$ & $95 \% \mathrm{CI}$ & Sig & AOR & $95 \% \mathrm{CI}$ & Sig \\
\hline \multicolumn{10}{|l|}{ Methotrexate } \\
\hline Yes & 1.31 & {$[0.91,1.88]$} & & 1.45 & {$[1.28,1.64]$} & $* * *$ & 1.43 & {$[1.28,1.61]$} & $* * *$ \\
\hline No & Ref & & & Ref & & & Ref & & \\
\hline \multicolumn{10}{|c|}{ Other DMARDS ${ }^{\dagger}$} \\
\hline Yes & 0.92 & {$[0.68,1.24]$} & & 1.17 & {$[1.05,1.30]$} & $* *$ & 1.14 & {$[1.03,1.26]$} & * \\
\hline No & Ref & & & Ref & & & Ref & & \\
\hline \multicolumn{10}{|c|}{ Glucocorticoids } \\
\hline Yes & 0.62 & {$[0.44,0.88]$} & $* *$ & 0.67 & {$[0.60,0.76]$} & $* * *$ & 0.67 & {$[0.60,0.75]$} & $* * *$ \\
\hline No & Ref & & & Ref & & & Ref & & \\
\hline \multicolumn{10}{|l|}{ Opioids } \\
\hline Yes & 0.96 & {$[0.68,1.35]$} & & 0.81 & {$[0.72,0.90]$} & $* * *$ & 0.82 & {$[0.74,0.91]$} & $* * *$ \\
\hline No & Ref & & & Ref & & & Ref & & \\
\hline \multicolumn{10}{|c|}{ External Environment Characteristics } \\
\hline \multicolumn{10}{|l|}{ Region } \\
\hline East & Ref & & & Ref & & & Ref & & \\
\hline Midwest & 1.20 & {$[0.76,1.89]$} & & 1.01 & {$[0.86,1.19]$} & & 1.03 & {$[0.89,1.20]$} & \\
\hline South & 1.17 & {$[0.75,1.84]$} & & 0.84 & {$[0.71,0.98]$} & $*$ & 0.87 & {$[0.75,1.01]$} & \\
\hline West & 1.16 & {$[0.64,2.11]$} & & 0.99 & {$[0.79,1.24]$} & & 1.01 & {$[0.82,1.25]$} & \\
\hline \multicolumn{10}{|l|}{ Index Year } \\
\hline 2010 & Ref & & & Ref & & & Ref & & \\
\hline 2011 & 0.95 & {$[0.47,1.92]$} & & 0.88 & {$[0.69,1.11]$} & & 0.88 & {$[0.71,1.11]$} & \\
\hline 2012 & 0.89 & {$[0.44,1.76]$} & & 0.82 & {$[0.65,1.04]$} & & 0.83 & {$[0.66,1.03]$} & \\
\hline 2013 & 0.96 & {$[0.48,1.94]$} & & 0.89 & {$[0.70,1.13]$} & & 0.90 & {$[0.71,1.12]$} & \\
\hline 2014 & 1.02 & {$[0.48,2.15]$} & & 0.9 & {$[0.70,1.15]$} & & 0.91 & {$[0.72,1.15]$} & \\
\hline
\end{tabular}

Note: Based on 6,970 adults with Rheumatoid Arthritis aged between 18 and 63 as of the index date, initiated on Tumor Necrosis Factor Inhibitors between 2010 and 2014, with continuous enrollment for 12 months baseline and 12 months follow-up periods in a commercial insurance plan.

Asterisks represent significant group differences compared to the reference group.

$\dagger$ include Sulfasalazine, Leflunomide, Hydroxychloroquine, Azathioprine, Chloroquine, Cyclophosphamide, Cyclosporine, Minocycline, and Sodium Aurothiomalate.

DMARDS: Disease modifying anti-rheumatic drugs; ER: Emergency room; HMO: Health maintenance organization; PPO: Preferred provider organization; IP: Inpatient; NSAIDS: Nonsteroidal anti-inflammatory drugs.

$* * * \mathrm{p}<.001 ; * * .001 \leq \mathrm{p}<.01 ; * .01 \leq \mathrm{p}<.05$. 
Table 3.4. Contribution of Individual Characteristics to the Observed Difference in TNFi Treatment Response Rate by Depression Status

Working-age Adults with Rheumatoid Arthritis Initiated on TNFi QuintilesIMS Real-World Data Adjudicated Claims Database, 2009 - 2015

\begin{tabular}{|c|c|c|c|}
\hline \multirow{2}{*}{$\begin{array}{l}\text { Variables } \\
\text { [a] }\end{array}$} & \multirow{2}{*}{$\begin{array}{l}\text { Value in Percentage Points } \\
\text { [b] }\end{array}$} & \multirow{2}{*}{$\begin{array}{l}\text { Standard } \\
\text { Errors } \\
{[c]}\end{array}$} & \multirow{2}{*}{$\begin{array}{l}\text { \% Total } \\
\text { [d] }\end{array}$} \\
\hline & & & \\
\hline \multicolumn{4}{|l|}{ Explained by independent variables } \\
\hline \multicolumn{4}{|c|}{$\begin{array}{l}\text { Differences in Predisposing Characteristics } \\
\end{array}$} \\
\hline Sex & 0.41 & 0.0020 & $5.23 \%$ \\
\hline Age & 0.17 & 0.0004 & $2.21 \%$ \\
\hline \multicolumn{4}{|c|}{ Differences in Enabling Characteristics } \\
\hline Insurance Plan Type & -0.05 & 0.0003 & $-0.68 \%$ \\
\hline \multicolumn{4}{|c|}{ Differences in Need Characteristics } \\
\hline Number of Chronic Physical Conditions & -0.07 & 0.0011 & $-0.84 \%$ \\
\hline Substance Abuse & 0.05 & 0.0008 & $0.66 \%$ \\
\hline Anxiety & 1.52 & 0.0055 & $19.22 \%$ \\
\hline \multicolumn{4}{|c|}{ Differences in Health Services Utilization } \\
\hline Any Emergency Department Visit & 0.46 & 0.0024 & $5.78 \%$ \\
\hline Any Inpatient Visit & -0.14 & 0.0023 & $-1.73 \%$ \\
\hline \multicolumn{4}{|c|}{ Differences in Medication Use } \\
\hline NSAIDS & -0.17 & 0.0007 & $-2.16 \%$ \\
\hline Methotrexate & -0.29 & 0.0005 & $-3.72 \%$ \\
\hline Other DMARDS & -0.27 & 0.0009 & $-3.43 \%$ \\
\hline Glucocorticoids & 0.44 & 0.0007 & $5.55 \%$ \\
\hline Opioids & 0.97 & 0.0026 & $12.31 \%$ \\
\hline \multicolumn{4}{|c|}{ Differences in External Environment Characteristics } \\
\hline Region & -0.19 & 0.0006 & $-2.36 \%$ \\
\hline Index Year & 0.08 & 0.0006 & $1.07 \%$ \\
\hline Total explained portion & 2.93 & & $37.12 \%$ \\
\hline $\begin{array}{l}\text { Unexplained portion } \\
\text { Difference in Treatment Response Rate } \\
\text { between Depression and No Depression }\end{array}$ & 4.97 & & $62.88 \%$ \\
\hline Group & 7.90 & & $100.0 \%$ \\
\hline
\end{tabular}

Note: Based on 6,970 adults with Rheumatoid Arthritis aged between 18 and 63 as of the index date, initiated on Tumor Necrosis Factor Inhibitors between 2010 and 2014, with continuous enrollment for 12 months baseline and 12 months follow-up periods in a commercial insurance plan.

The decomposition is based on pooled weights. The pooled weights were derived from parameter estimates of the logistic regression on treatment response, using pooled data from both depression and no depression groups.

Column [d] gives the percentage of the difference in treatment response by depression explained by differences in each independent variable between the two groups 


\section{CHAPTER 4}

\section{Tumor Necrosis Factor Inhibitor Therapy and the Risk of Developing Depression among Working Age Adults with Rheumatoid Arthritis}

\subsection{Abstract}

Background. Individuals with Rheumatoid Arthritis (RA) have high risk of developing depression because of the overall burden of systemic inflammation. Effective treatment with anti-inflammatory drugs specifically, tumor necrosis factor inhibitors (TNFi) may therefore reduce the risk of developing depression by lowering the inflammatory burden and improving health status in RA patients. Objective. Evaluate the association between TNFi therapy and the risk of developing depression among working age adults with RA. Method. A retrospective observational cohort study design was used with data derived from commercial claims data from QuintilesIMS RWD Adjudicated Claims for the period October 1, 2009 to September 30, 2015. Working-age adults (18- 62 years) with RA, who were initiated on TNFi therapy and were continuously enrolled during the observation periods (one-year baseline, one-year treatment and one-year follow-up periods) were included $(\mathrm{N}=4,222)$. Treatment response to TNFi was measured using prescription drug claims based on published validated algorithm. Multivariable logistic regression was used to examine the association between treatment response to TNFi therapy and the risk of developing depression, after controlling for baseline demographic characteristics, clinical characteristics and RA related medication use. To control for the observable differences in the characteristics of the TNFi responders vs. TNFi non-responders, inverse probability of treatment weighting technique (IPTW) was used. Results. Overall, 8.5\% developed depression during the follow-up period and $39.8 \%$ responded to TNFi treatment during the one-year treatment period. A lower percentage of TNFi responders developed 
depression $(7.1 \%$ vs. $9.4 \%, \mathrm{p}<.005)$ as compared to TNFi non-responders. After controlling for other risk factors, TNFi responders were $20 \%$ less likely to develop depression during the follow-up period $(\mathrm{AOR}=0.80,95 \% \mathrm{CI}=0.64,0.98)$ as compared to TNFi non-responders.

Conclusion. Effective treatment with TNFi therapy reduces the risk of depression in working-age adults with RA.

\subsection{Introduction}

Rheumatoid arthritis (RA) is a chronic systemic inflammatory condition that negatively impacts both physical and mental health of an individual ${ }^{125,126}$. Adults with RA are at higher risk of developing depression because of the overall burden of systemic inflammation ${ }^{15,127}$. Studies have reported increased risk of developing depression after RA diagnosis ${ }^{16}$. The incident rate of depression in RA patients is higher than non-RA patients ${ }^{17}$.

The biological plausibility of the link between RA, depression and inflammation stems from the robust association between inflammation and depression ${ }^{63}$. It has been reported that RA patients with depression have significantly elevated levels of inflammatory biomarkers such as TNF- $\alpha$, c-reactive protein (CRP) as compared to RA patients without depression ${ }^{12,128-130}$. Human experimental studies have demonstrated that administration of pro-inflammatory cytokines in healthy individuals triggers depressive symptoms such as mood disturbances, anhedonia, anorexia and sleep disturbance ${ }^{13,14}$.

Risk of depression in RA patients is worrisome because of the negative effects of depression on the patient. For example, depression increases the risk of mortality by more than 2 folds ${ }^{73}$, worsens health related quality of life ${ }^{131}$, reduces adherence and treatment response to anti-rheumatic drugs ${ }^{32,48}$ and increases healthcare resource utilization and costs ${ }^{47,74}$. 
Therefore, examining factor that reducing the risk of depression among patients with RA is important. The inflammatory process that affect both depression and RA may represent a unique opportunity to reduce the risk of depression in RA patients. For example, clinical trials have provided some evidence on the efficacy of anti-inflammatory agents in reducing depressive symptoms ${ }^{132}$. In this context, specific drugs used for preventing RA progression may be of particular significance. Drugs inhibiting TNF- $\alpha$ (TNFi) have been proven to be highly efficacious in reducing inflammation and preventing disease progression in $\mathrm{RA}^{56}$. Due to the positive association between depression and inflammation, it is plausible that drugs inhibiting TNF- $\alpha$ (TNFi) may reduce the risk of developing depression in RA patients ${ }^{68}$. Some randomized controlled and open label trials have demonstrated that TNFi therapy improves depressive symptoms and quality of life in RA patients ${ }^{133-135}$. However, in real world clinical practice only one-third of RA patients respond to TNFi therapy ${ }^{136}$. Therefore, the beneficial effect of TNFi on depression may be limited to RA patients who respond to TNFi therapy. The effect of treatment response to TNFi on the risk of developing depression remains unclear from previous studies ${ }^{133-}$ 135 .

Furthermore, depression in RA has significant implications on working age adults (18-64 years) because of the illness burden. As the onset of both RA and depression occurs in individuals during the most productive mid-life years of lives ${ }^{50,137}$, the cumulative impact of RA and depression on productivity loss due to missed work days, work disability and loss of employment among working age adults is concerning ${ }^{100,138}$. Therefore, it is important to understand pathways that can reduce the risk of developing depression in working-age adults with RA. No published studies have specifically evaluated treatment response to TNFi therapy on risk of developing depression in this population. 
Therefore, the objective of this study was to evaluate the treatment response to TNFi on the risk of developing depression in working age RA patients who are cared for in real world practice setting using administrative claims data. We hypothesize that RA patients who respond to TNFi therapy will be significantly less likely to develop depression as compared to RA patients who did not respond to TNFi therapy.

Conceptual Framework: The conceptual framework for this study was adapted from the determinants of a health and chronic disease model proposed by Wilkinson and Marmot ${ }^{70}$. This model delineates the relationship between community resources, access to care variables, individual physical make-up, biological risk factors, and treatment factors that affect the incidence of chronic conditions.

\subsection{Methods}

\section{Data}

We used data from QuintilesIMS Real World Data Adjudicated Claims for the period October 1, 2009 to September 30, 2015. This is an integrated claims database that includes enrollment, medical and pharmacy claims information for more than 95 million enrollees of commercial plans across the US. The data includes the records of $90 \%$ of hospitals, $80 \%$ of doctors and $85 \%$ of large companies in the US. This database also records enrollees demographics (year of birth, gender, geographic region), plan type (health maintenance organization, preferred provider organization), payer type (commercial, self-insured), prescription drug information and considered nationally representative of commercially insured individuals less than 65 years of age in the US.

\section{Study Design}


A retrospective observational cohort study design was used. The index date was defined as the first observed prescription date for a TNFi biologic between October 2010 and September 2013. The study period was divided into 12 months pre-index or baseline period, 12 months treatment period during which the treatment response to TNFi was measured and 1-year followup period when risk of developing depression was assessed.

\section{Study Cohort - Depression Free RA Patients}

The study cohort consisted of adults aged between 18 and 62 years, with a diagnosis of RA in the baseline period and initiated on TNFi therapy between April 2010 and September 2013 (Supplemental Figure 7.1.2). Continuous enrollment for the 12 months baseline period, 12 months treatment period and 12 months follow up period was required. Adults with prevalent depression during the baseline and treatment period were excluded. In addition, adults diagnosed with other autoimmune conditions for which biologics are used (crohn's disease, ulcerative colitis, ankylosing spondylitis, psoriasis, psoriatic arthritis, juvenile idiopathic arthritis) during the baseline or treatment or follow-up period were excluded. Furthermore, adults diagnosed with Non-Hodgkin's lymphoma, chronic lymphocytic leukemia or HIV/AIDS were also excluded (Supplemental Figure 7.1.2).

\section{Measures}

\section{Dependent variable: Newly-diagnosed Depression (Yes/No)}

Depression in the follow-up period was identified using a standard algorithm ${ }^{111,112}$. Adults with ICD-9-CM codes of 296.2 (major depressive disorder, single episode), 296.3 (major depressive disorder, recurrent episode), 298.0 (depressive type psychosis), 300.4 (neurotic depression), 309.1 (prolonged depressive reaction), and 311 (depressive disorder, not classified) during the follow-up period were considered to have developed depression. 
Key Independent Variable: Response to Treatment with TNFi (Yes/No)

Treatment response to TNFi was measured using the claims based effectiveness algorithm developed by Curtis et al. ${ }^{109}$. Curtis et al. used the Veteran Health Administration (VHA) claims database to operationalize treatment response to biologics and validated it using the gold-standard for treatment response (i.e. DAS28) from the VHA Rheumatoid Arthritis registry ${ }^{109}$. The investigators tested the performance of the algorithm in claims database of commercially insured individuals ${ }^{110}$. A high positive predictive value of $87 \%$ was found between claims-based algorithim and the gold standard measure of treatment response i.e change in DAS28 ${ }^{110}$. Using this algorithm, treatment response to TNFi was measured as a dichotomous variable (Yes/No) during a one-year treatment period (one-year after the index date), conditional on meeting all the following six criteria:

(1) Adherence to index TNFi: Adherence to TNFi was calculated using proportion of days covered (PDC). The PDC was calculated by using the date of service and the day supply for each fill of the index TNFi. Patients with a PDC of at least $80 \%$ were considered adherent. Patients taking infliximab, were considered adherent if they had at least 7 infusions in the 1-year treatment period.

(2) No increase in TNFi dose compared to the first dose: Dose increases were measured based on the type of TNFi medications. For Adalimumab (subcutaneous) users, the dose escalation criteria required no more than $40 \mathrm{mg}$ per week; for Certolizumab pegol (subcutaneous) users the dose escalation criteria was $\geq 200 \mathrm{mg}$ (after 56 days) of the index-dose; for Etanercept (subcutaneous) users the dose escalation criteria was $\geq 100 \mathrm{mg} / \mathrm{week}$ of the index dose; for Golimumab (intravenous) users the dose escalation criteria was $\geq 50 \mathrm{mg}$ from the first to last dose; for Golimumab (subcutaneous) users the dose escalation criteria was $\geq 25 \mathrm{mg} /$ week post- 
index (i.e., $>50 \mathrm{mg}$ monthly post-index); for Infliximab users the dose escalation criteria were $\geq 100 \mathrm{mg}$ from the first to last dose or $<11$ infusion dates post-index or $\geq 7$ weeks between doses (after the third dose).

(3) No new conventional synthetic DMARD: No addition of methotrexate, sulfasalazine, leflunomide, or hydroxychloroquine in the treatment period in RA patients who did not have any claims for that drug in the baseline period.

(4) No switch to another biologic drug approved for use in patients with RA.

(5) No new/increased oral glucocorticoid: For patients with no pre-index oral glucocorticoids, $<30$ days of oral glucocorticoids between the index dates and 90 days post-index; for patients with pre-index oral glucocorticoids, a cumulative post-index dose within $120 \%$ of the cumulative pre-index dose.

(6) Fewer than 2 glucocorticoid injections (No more than one parenteral or intra-articular glucocorticoid joint injection on unique days) in months 4 to 12 of the post-index period.

\section{Other independent variables:}

Other independent variables measured during the baseline period included the demographic characteristics such as age, sex, region(East, Midwest, South, and West), insurance and insurance plan type (health maintenance organizations [HMOs]; preferred provider organizations (PPOs); clinical characteristics included total number of chronic physical conditions, anxiety and substance abuse; healthcare utilization (any inpatient and any ED visit), and RA related medication use (glucocorticoids, DMARDs, NSAIDs and opioids). Total number of chronic physical conditions was measured using the Clinical Classifications Software managed by the Agency for Healthcare Research and Quality (AHRQ).

\section{Statistical Analysis}


Chi-square and adjusted odds ratios (AOR) and 95\% Confidence intervals (CI) from multivariable logistic regression analyses were used to examine the association between treatment response to TNF and newly diagnosed depression after controlling for baseline demographic characteristics, clinical characteristics and RA related medication use. As the rate of newly diagnosed depression was less than $10 \%$, adjusted odds ratio can be used to approximate relative risk ${ }^{139}$. Therefore, we used the term risk and odds ratios interchangeably. Accounting for observable selection bias: To control for the observable differences in the baseline characteristics of the TNFi responders vs. non-responders, inverse probability weighting technique (IPTW) was used. This technique gives weight to each individual based on the inverse of their probability of being in the responder group. Thus, individuals who have lower probability of being in the responder group were up-weighted, and those with higher probability of being in the responder group were down weighted. This helped to balance the probability of being in the responder group vs the non-responder group. To derive the IPTW weights, logistic regression was at first conducted on treatment response to TNFi and probabilities of being in the responder vs. non-responder group was calculated, the weights were created using inverse of probabilities. To account for the differences in group sizes, the weights were further stabilized by dividing them with sample size of each group. All analyses were conducted using SAS 9.4 ${ }^{140}$

\subsection{Results}

Out of the 4,222 working-age adults with RA who were initiated on TNFi, and were depression free during the baseline and treatment period, $75 \%$ were female, with a mean age of 50 years; $45.4 \%$ were living in the Southern region of the US; $59 \%$ suffered from 1 to 3 additional chronic condition; $73.5 \%$ were on Methotrexate and 55.1\% were on NSAIDS during the baseline period (Appendix 7.2). 


\section{Response to TNFi Treatment:}

Based on the algorithm, 39.8\% (N=1,679) were classified as responders of TNFi treatment during the one-year treatment period after index date (Table 4.1). The most common reason for non-response to TNFi therapy was low adherence to index TNFi (42\%).

There were statistically significant differences in the baseline characteristics of the TNFi responders and TNFi non-responders in sex, age, insurance, region, insurance plan type and use of RA related medications in the baseline (Table 4.2). For example, a significantly higher proportion of adults in the TNFi non-responder group used glucocorticoids (75\% vs. 67\%) and opioids (59\% vs. 52\%). Conversely, the use of Methotrexate in the baseline period was lower in the TNFi non-responder group as compared to the TNFi responder group (76\% vs. $71 \%)$. After the application of IPTW, such observable differences were no longer significant as shown in Table 4.2.

Overall, $8.5 \%$ developed depression during the follow-up period. A lower percentage of TNFi responders developed depression during the one-year follow-up period after treatment as compared to TNFi non-responders ( $7.1 \%$ vs. $9.4 \%$; $p<.005)$ After controlling for potential confounders, we found that TNFi responders were $20 \%$ less likely to develop depression as compared to TNFi non-responders with AOR $=0.80$ and 95\% CI $=0.64,0.98$ (Table 4.3).

Other baseline risk factors for depression included female $\operatorname{sex}(\mathrm{AOR}=2.12 ; 95 \% \mathrm{CI}=1.64$, 2.74), number of chronic conditions ( $>3$ vs. 0 : $\mathrm{AOR}=1.97 ; 95 \% \mathrm{CI}=1.34,2.88$ ), opioid use $(\mathrm{AOR}=2.07 ; 95 \% \mathrm{CI}=1.69,2.52)$ and emergency department visit $(\mathrm{AOR}=1.32 ; 95 \% \mathrm{CI}=1.04$, 1.68). 


\subsection{Discussion}

In a depression free cohort of working age RA patients treated with TNFi, we found that the overall rate of newly diagnosed depression was $8.5 \%$. In a previous study done in 83 Turkish patients with RA treated in an outpatient rheumatology clinic, researchers reported a markedly lower prevalence of depressive disorders among patients treated with TNFi (6.3\%) as compared to those treated with other drugs $(41.8 \%)^{141}$. Most epidemiological studies in RA patients have examined the prevalence of depression in RA patients ${ }^{15}$, and only a handful of studies examined the incidence of depression in RA patients ${ }^{16,17}$. A systematic review of 72 studies in RA patients, reported a $16.8 \%$ prevalence of major depressive disorders ${ }^{15}$. In one UK study, approximately $30 \%$ of RA patients developed depression within 5 years of RA diagnosis ${ }^{16}$. Therefore, in light of these previous findings, the rate of depression in RA patients treated with TNFi appears to be lower. In order to determine whether the lower rate of depression is a direct effect of treatment with TNFi or whether it could be attributed to improvement in RA disease secondary to treatment, future studies need to also incorporate control population of RA patients treated with other antirheumatic regimens such as DMARDS.

A noteworthy finding of our study is that treatment response to TNFi therapy reduced the risk of developing depression by $20 \%$, some plausible explanations for this finding is that patients who respond to TNFi therapy may have improved RA-related outcomes that may lower the risk of developing psychological problems including depression. TNFi therapy may also suppress inflammation and lower the risk of developing subsequent depression ${ }^{12,127}$.

The reduction in the risk of depression in TNFi responders has clinical implications for treatment of RA as well as depression. Our findings suggest that intervention with TNFi therapy may not only improve RA-related outcomes, but also reduce the risk of developing depression. Our study findings offer the possibility of including anti-inflammatory agents in treating existing 
depression and testing whether including anti-inflammatory agents in depression treatment regimens alleviates depressive symptoms. Future studies need to evaluate the direct effect of TNFi treatment on the levels of inflammatory cytokines among RA patients with comorbid depression.

In our study population of depression free adults with RA, 39.8\% responded to TNFi therapy. The response rate to TNFi in this sub group is slightly higher as compared to response rate (DAS-28 based response criteria) to TNFi observed in RA population in previous studies using registry and administrative claims data. These differences in the analysis could be attributed to differences in databases as well as the sub-population used. Our study excluded patients with depression. It has been documented that adults with depression have lower rates of response to TNFi therapy as compared to those without depression ${ }^{142}$. One study using a Danish registry data reported a response rate of $25 \%$ to $\mathrm{TNFi}^{143}$. Curtis et al. using Veterans Health Administration database reported a response rate of $27 \%$ to TNFi therapy based on algorithm defined treatment response ${ }^{109}$. Other studies using the same algorithm on different databases such as Medco Health Solutions Pharmacy Benefit Manager Database ${ }^{144}$ and Texas Medicaid database $^{145}$ reported $32 \%$ and $15.7 \%$ response rate respectively with TNFi therapy in RA patients.

Our study findings showed that the major reason for non-response to TNFi therapy was low adherence, with about $42 \%$ of the RA patients having low adherence to TNFi. This finding is consistent with previous studies on RA patients that also highlighted non-adherence to TNFi as one of the major contributing factor for poor response to TNFi therapy ${ }^{109,144-146}$. For example, in a prospective multicenter large cohort study conducted in UK, researchers demonstrated that self-reported non-adherence to TNFi was a strong predictor of poor response to TNFi therapy, 
independent of socio demographic and clinical characteristics of the patients ${ }^{146}$. Therefore, physicians should encourage patients to adhere to RA treatment regimens and make them aware that low adherence to TNFi medications adversely affect treatment response.

This study has many strengths. First it adds to the nascent literature on how treatment response to antirheumatic therapy may have potential benefit in reducing psychiatric comorbidities such as depression in RA patients. Other strengths include the use of the nationally representative data of commercially insured working age adults (18 to 63 years), use of longitudinal study design to track individuals across different providers, settings over a period of 3 years, availability of prescription drug claims, ability to measure treatment response with readily available claims data, large sample size, high validity and complete information of prescription drugs and the use of statistical techniques to control for unobserved selection bias.

The findings of this study should be interpreted considering the potential limitations that include the lack of severity measures for chronic conditions and lack of information on individual characteristics such as race, body mass index, exercise and smoking status that may predict depression. Furthermore, the study findings are not generalizable beyond commercially insured working age adults. Also because of the nature of claims data we can only observe prescription of medications and not actual use of medications. Other limitations include selection bias due to unobserved variables and underdiagnoses of depression in claims data.

\subsection{Conclusion}

In summary, RA patients who responded to TNFi were less likely to develop depression as compared to RA patients who did not respond to TNFi therapy. This finding highlights the need of optimal treatment response to TNFi for attenuating the heightened risk of depression 
associated with RA. Prospective clinical and population based registry studies are needed to investigate the role of inflammation in the pathogenesis of depression in patients with RA.

\subsection{Acknowledgements and Data User Statement}

\section{Funding}

Research reported in this was supported by the NIGMS of the National Institutes of Health under award number U54GM104942. The content is solely the responsibility of the authors and does not necessarily represent the official views of the National Institutes of Health.

\section{Data User Statement}

The statements, findings, conclusions, views, and opinions contained and expressed in this article are based in part on data obtained under license from the following QuintilesIMS information services: QuintilesIMS Real-World Data Adjudicated Claims - US, October 2009September 2015, QuintilesIMS Health Incorporated. All Rights Reserved. The statements, findings, conclusions, views, and opinions contained and expressed herein are not necessarily those of QuintilesIMS Health Incorporated or any of its affiliated or subsidiary entities. 
Table 4.1. Components of the Algorithm for TNFi Response Status at 1 year after TNFi Initiation $(\mathrm{N}=4,222)$

Low adherence to index TNFi $(\mathrm{PDC}<0.8)$

Switched to new biologics

$18.8 \%$

Dose escalation of TNFi

$9.2 \%$

Conventional DMARD addition after index

Dose increase of oral glucocorticoid

$>1$ glucocorticoid joint injection

Note: Based on 4,222 working age adults with RA, initiated on Tumor Necrosis Factor Inhibitors between 2010 and 2013, aged between 18 and 62, with continuous enrollment for 3 years in a commercial insurance plan. Individual components of TNFi response criteria will not add to $39.8 \%$ because an individual may satisfy one component of the algorithm, while failing to satisfy another component of the algorithm. 
Table 4.2. Selected Baseline Characteristics of TNFi Responders vs. TNFi Non-Responders

\begin{tabular}{|c|c|c|c|c|c|c|}
\hline & \multicolumn{3}{|c|}{ Prior to IPTW } & \multicolumn{3}{|c|}{ After applying IPTW } \\
\hline & Responder & Non-Responder & & Responder & Non-Responder & \\
\hline & Col\% & Col\% & Sig & Weighted Col\% & Weighted Col\% & Sig \\
\hline Sex & & & 0.04 & & & 0.87 \\
\hline Female & 74.6 & 76.8 & & 74.0 & 74.2 & \\
\hline Male & 25.4 & 23.2 & & 26.0 & 25.8 & \\
\hline Age & & & $<.001$ & & & 0.98 \\
\hline $18-34$ years & 7.4 & 9.6 & & 8.6 & 8.6 & \\
\hline $35-44$ years & 15.1 & 17.5 & & 16.6 & 16.5 & \\
\hline $45-54$ years & 36.0 & 35.8 & & 35.8 & 35.9 & \\
\hline $55-62$ years & 41.4 & 37.1 & & 39.0 & 39.0 & \\
\hline Region & & & 0.002 & & & 0.65 \\
\hline East & 18.0 & 17.0 & & 17.2 & 17.3 & \\
\hline Midwest & 31.9 & 28.6 & & 29.9 & 29.8 & \\
\hline South & 41.2 & 46.0 & & 44.7 & 44.7 & \\
\hline West & 8.9 & 8.4 & & 8.2 & 8.2 & \\
\hline Insurance Plan Type & & & $<.001$ & & & 0.98 \\
\hline HMO & 9.7 & 10 & & 10.0 & 10.0 & \\
\hline PPO & 76.0 & 78.8 & & 77.9 & 77.9 & \\
\hline Other & 14.3 & 11.2 & & 12.1 & 12.1 & \\
\hline NSAIDS & & & 0.420 & & & 0.98 \\
\hline Yes & 56.3 & 55.3 & & 55.4 & 55.3 & \\
\hline No & 43.7 & 44.7 & & 44.6 & 44.7 & \\
\hline Methotrexate & & & $<.001$ & & & 0.68 \\
\hline Yes & 76.4 & 70.7 & & 72.6 & 72.5 & \\
\hline No & 23.6 & 29.3 & & 27.5 & 27.5 & \\
\hline Other DMARDS $\dagger$ & & & 0.240 & & & 0.95 \\
\hline Yes & 51.0 & 49.5 & & 49.2 & 49.2 & \\
\hline No & 49.0 & 50.5 & & 50.8 & 50.8 & \\
\hline Glucocorticoids & & & $<.001$ & & & 0.99 \\
\hline Yes & 67.4 & 75.0 & & 71.8 & 71.7 & \\
\hline No & 32.6 & 25.0 & & 28.2 & 28.3 & \\
\hline Narcotics & & & $<.001$ & & & 0.99 \\
\hline Yes & 51.9 & 58.8 & & 54.1 & 54.0 & \\
\hline No & 48.1 & 41.2 & & 45.9 & 46.0 & \\
\hline
\end{tabular}

Note: Based on 4,222 working age adults with RA, initiated on Tumor Necrosis Factor Inhibitors between 2010 and 2013, aged between 18 and 62, with continuous enrollment for 3 years in a commercial insurance plan. $\dagger$ Other DMARDS include Sulfasalazine, Leflunomide, Hydroxychloroquine, Azathioprine, Chloroquine, Cyclophosphamide, Cyclosporine, Minocycline, and Sodium Aurothiomalate. 
Table 4.3. Adjusted Odds Ratio and 95\% Confidence Intervals of Selected Significant Variables from Logistic Regression on Newly Diagnosed Depression among Working Age Adults with RA initiated on TNFi QuintilesIMS Real-World Data Adjudicated Claims Database

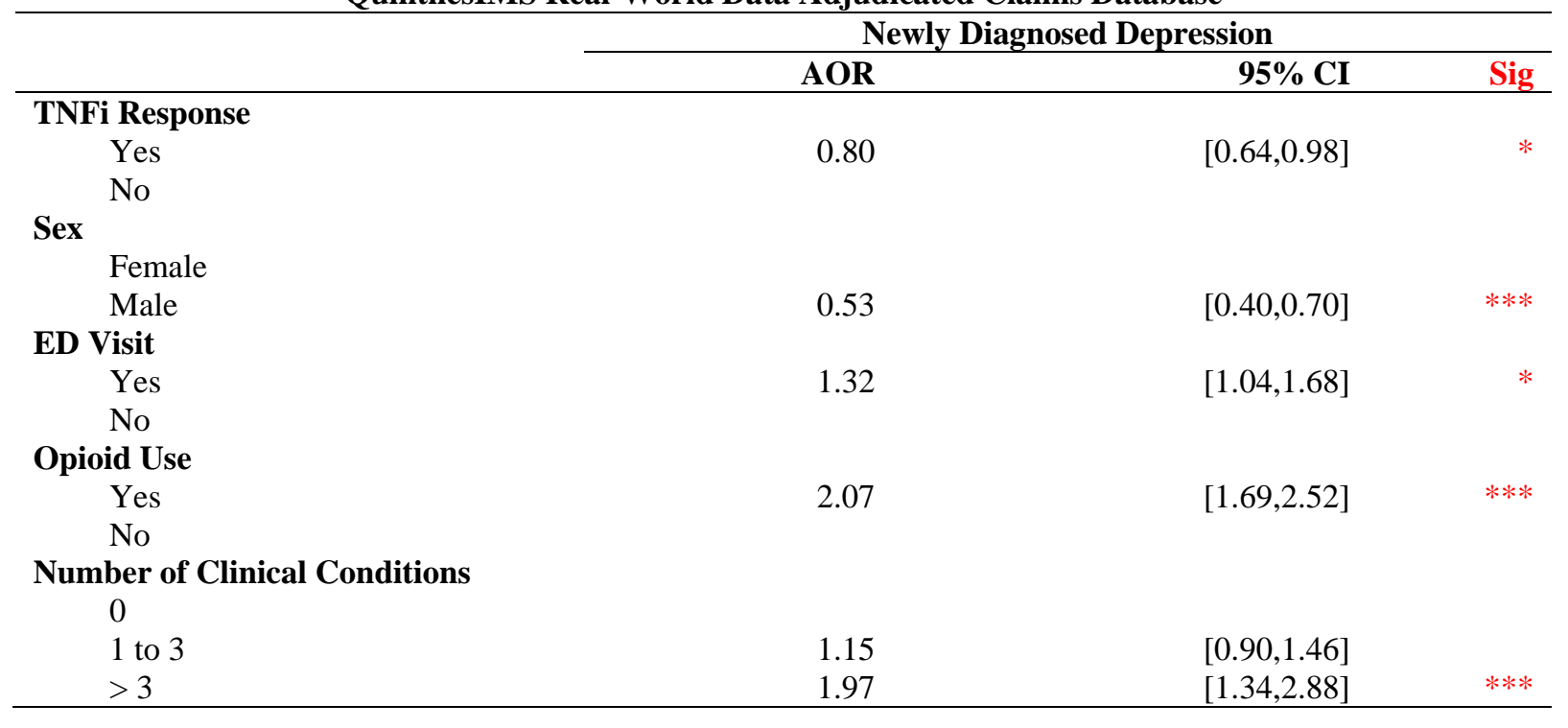

Note: Based on 4,222 working age adults with RA, initiated on Tumor Necrosis Factor Inhibitors between 2010 and 2013, aged between 18 and 62, with continuous enrollment for 3 years in a commercial insurance plan. AOR= Adjusted Odds Ratio, CI= Confidence Interval. $* * * \mathrm{p}<.001 ; * * .001 \leq \mathrm{p}<.01 ; * .01 \leq \mathrm{p}<.05$. Other factors that were adjusted in the model included age, region, insurance plan type, index year, inpatient visit, anxiety, substance abuse, glucocorticoid use, methotrexate use, other disease modifying antirheumatic drugs use, non-steroidal antiinflammatory drug use and narcotic use. 


\section{CHAPTER 5}

\section{Summary and Conclusion}

\subsection{Summary}

Understanding depression as a comorbidity in medical conditions is very important because depression has been identified as the second leading cause of disability that imposes the highest burden on working-age population ${ }^{147}$. The World Health Survey reported that depression leads to the greatest decrement in health compared to any chronic physical conditions such as angina, asthma, arthritis and diabetes as reported by the World Health Organization ${ }^{148}$. Again, data from the National Comorbidity Survey Replication, a nationally representative household survey in the U.S. have shown that the overall burden of functional disability is significantly higher with depression than with chronic medical disorders ${ }^{149}$. The burden of depression is significant because almost $80 \%$ of individuals with depression have some level of functional impairment due to depression ${ }^{150}$.

The link between RA and depression is very complex and influenced by a multitude of factors such as shared pathophysiologic pathways of inflammation, RA-related treatment response, as well as the patient's demographic and socio-economic factors, and general difficulties in coping with the disease. Depression can increase the clinical, humanistic, and economic burden among RA patients. Growing evidence now suggest that depression is a systemic inflammatory condition and may exert negative influence on the treatment response in RA by its effect on pro-inflammatory cytokines. Therefore, it is important to understand how depression is related to the treatment effectiveness of specific cytokine inhibitor drugs such as Tumor Necrosis Factor Inhibitor (TNFi) therapy that are used for the treatment of RA. The powerful anti-inflammatory effect of TNFi may have a potential beneficial effect in reducing the 
risk of depression in RA patients. In real-world clinical practice only one-third of RA patients respond to TNFi therapy (Curtis, Schabert et al. 2014). Therefore, it is important to assess the relationship between treatment response to TNFi and the risk of developing depression.

However, a comprehensive examination of how depression affects RA and how RA can affect depression in real-world clinical practice settings is lacking. As the onset of both RA and depression occurs during the prime working years of lives, it is important to analyze these issues in working-age adults with RA. Therefore, this dissertation had three related aims focusing on working-age adults: 1) estimating the illness burden of depression in RA; 2) how depression can affect RA treatment response; and 3) how RA treatment response can affect depression. The study used data from multiple nationally representative sources to triangulate the complex relationship between RA and depression in working-age adults. These data sources were: the nationally representative Medical Expenditure Panel Survey (MEPS) for years 2009, 2011, 2013, and 2015 and a retrospective claims database for commercially insured working-age adults for the years 2009 through 2015.

\section{Illness Burden of Depression in RA}

We found that one in every four working-age adults reported prevalent depression. Presence of depression in RA patients was associated with excess clinical, humanistic and economic burden. For example, depression was associated with severe pain related interference with normal work, lower HRQoL scores in the mental health domain, higher annual healthcare expenditures and out-of-spending burden. Compared to RA patients without depression, adults with RA and depression were significantly more likely to be unemployed and among employed adults those with depression had a significantly higher number of missed work days and higher 
lost wages due to missed work days. These findings underscore the magnitude of clinical, humanistic and economic burden associated with depression among working-age adults with RA. The interaction between Depression and RA treatment response

Depression was independently associated with treatment response to TNFi, even after adjusting for baseline predisposing, enabling, need and external environment factors among working-age RA patients. Among RA patients with depression, nearly three in every ten patients responded to TNFi therapy, whereas among RA patients without depression, nearly four in every ten patients responded to TNFi therapy. Post-regression non-linear decomposition analysis revealed that differences in baseline characteristics such as the presence of anxiety, glucocorticoid use and narcotic pain medication use, explained a substantial portion of the observed difference in treatment response to TNFi between RA patients with depression and without depression.

In addition, among RA patients without baseline depression, those who responded to TNFi therapy, were less likely to develop depression as compared to those who did not respond to TNFi therapy. Among RA patients who responded to TNFi therapy, seven out of every hundred patients developed depression, whereas, among RA patients who did not respond to TNFi therapy, nine out of every hundred patients developed depression. This finding suggests that effective treatment with TNFi reduces the risk of depression among RA patients.

\subsection{Implications}

This dissertation research revealed that depression was associated with excess burden in many dimensions: pain-related work interference, health related quality of life, lost productivity, and healthcare costs among working-age adults with RA. The study findings have important implications for the employers because depression costs U.S. employers more than $\$ 31$ billion 
annually due to missed work and decreased work performance ${ }^{96}$. One study done in the U.S. reported that depression leads to the highest reduction in work performance and the highest employer burden relative to any other chronic conditions ${ }^{97}$.Strategies that employers may adopt to improve mental health in employees include organizing workplace health promotion programs and stress management projects, which have shown the benefits of prevention and management of depression in workplace ${ }^{98,99}$.

Our study findings have implications for clinicians and healthcare providers. It is welldocumented that depression in RA patients is often under recognized and under-treated in routine clinical practice (Hider, Tanveer et al. 2009, Margaretten, Julian et al. 2011). The current study also highlighted the negative effect of depression on treatment response to TNFi therapy. Our study underscores the need of incorporating depression screening and suggest the need developing effective clinical interventions for co-managing depression and RA in routine clinical practice. Potential strategies can include the integration of mental health services with rheumatology practice and facilitating mental health training for rheumatologists.

The reduction in the risk of depression in TNFi responders suggests that TNFi therapy is important not only for clinical improvement in RA disease but also for reducing the risk of depression. Therefore, aggressive and early treatment of RA with TNFi therapy may be needed to reduce the risk of developing depression, which may further improve treatment response. Thus, promoting the use of RA medications that has additional benefit in reducing the risk of depression in RA patients will help in reducing the excess burden of depression. Reducing the risk of depression may reduce overtreatment of RA with RA medications because depression is associated with poor treatment response and clinicians need to adjust RA treatment for those who are responding poorly to medications. Our study findings offer the possibility of including anti- 
inflammatory agents in treating existing depression and testing whether including antiinflammatory agents in depression treatment regimens alleviates depressive symptoms. Future studies need to evaluate the direct effect of TNFi treatment on the levels of inflammatory cytokines among RA patients with comorbid depression.

Future studies need to assess whether treatment of depression with antidepressants in collaborative care settings improve treatment response and RA-related clinical outcomes associated with particular anti-rheumatic therapies. Appropriate management of depression in RA would not only improve depression but also improve RA outcomes.

Our findings can inform payers and other stake-holders. Our study findings have important implications for the emerging trend that focus on "value-based" care, which emphasize on quality care at lower costs. These programs now require physicians to consider alternative payment models such as accountable care organizations, medical homes, bundled payments that emphasize quality and resource use. In all these programs, the payers and providers need to consider the case-mix of patients to benchmark expenditures and show value. Under bundled payment systems, a certain amount of prospective payment is made for episodes of care provided for a condition (example: depressive episodes in RA patients). Our estimate of excess direct medical expenditures associated with depression suggest that providers need to consider depression for risk adjustment, benchmarking expenditures, and in setting payments for episodes of care.

\subsection{Unique Contributions of this Study}

This study filled a critical knowledge gap. To the best of our knowledge, this is the first population based study that comprehensively examined the excess clinical, humanistic and economic burden of depression in working-age adults with RA. One of the unique contributions 
of this study is that it provided a nation-wide estimation of direct medical costs and productivity losses associated depression in working-age adults with RA.

By analyzing how treatment response to anti-rheumatic therapy may have the potential benefit in reducing psychiatric comorbidities such as depression in RA patients, our study findings add to the nascent literature on co-management of chronic physical and mental health conditions.

To understand the association between depression and treatment response, this study employed an econometric technique (i.e. decomposition). The decomposition technique quantified the contribution of individual patient characteristics in explaining the observed difference in treatment response to TNFi between RA patients with and without depression.

This study focused on patient experiences in with real world clinical settings. By examining the effect of mental health conditions (i.e. depression) in RA, this study highlights the complex relationship between mental health conditions and physical conditions. This study was more in line with real world experiences of the patients because the effectiveness of depression treatments were examined among individuals with multiple coexisting chronic physical conditions, a population often not included in clinical trials ${ }^{151}$.

\subsection{Strengths and Limitations}

The strengths of this study include the use of nationally representative datasets, use of longitudinal study design to track individuals across different providers, settings over a period of 3 years, availability of prescription drug claims, ability to measure the treatment response with readily available claims data, large sample size, the use of robust statistical techniques such as GLM, and recycled prediction in estimating the incremental costs and missed workdays, high validity and complete information of prescription drugs. 
Some of the potential limitations of our study include lack of severity measures RA and depression. However, by restricting adults who initiated TNFi therapy, we only included adults with moderate to severe RA. We did not have information on physiologic characteristics and lifestyle risk factors that may affect treatment response. Furthermore, the study findings of aim 2 and aim 3 are not generalizable beyond commercially insured working age adults. Also, because of the nature of claims data, we can only observe prescription fills and not actual use of medications. Other limitations include selection bias due to observable and unobserved variables and underdiagnoses of depression in claims data.

\subsection{Conclusion}

Depression is associated with excess illness burden in working-age adults with RA. RA treatment effectiveness is affected by depression and effective RA treatment reduced the risk of developing depression. Although this study filled a critical knowledge gap, future research needs to confirm our study findings by including RA disease activity, inpatient preferences, and personal health practices that may affect the relationship between treatment effectiveness and depression. Future studies need to evaluate whether treatment with the TNFi has direct effect on the levels of inflammatory biomarkers such as c-reactive protein (CRP), interleukins and tumor necrosis factors in patients with RA and depression. 


\section{References}

1. Myasoedova E, Crowson CS, Kremers HM, Therneau TM, Gabriel SE. Is the incidence of rheumatoid arthritis rising?: results from Olmsted County, Minnesota, 1955-2007. Arthritis and rheumatism. 2010;62(6):1576-1582.

2. Hunter TM, Boytsov NN, Zhang X, Schroeder K, Michaud K, Araujo AB. Prevalence of rheumatoid arthritis in the United States adult population in healthcare claims databases, 2004-2014. Rheumatology international. 2017.

3. van Vollenhoven RF. Sex differences in rheumatoid arthritis: more than meets the eye. BMC Medicine. 2009;7(1):12.

4. Crowson CS, Matteson EL, Myasoedova E, et al. The lifetime risk of adult-onset rheumatoid arthritis and other inflammatory autoimmune rheumatic diseases. Arthritis and rheumatism. 2011;63(3):633-639.

5. McInnes IB, Schett G. The Pathogenesis of Rheumatoid Arthritis. New England Journal of Medicine. 2011;365(23):2205-2219.

6. Cutolo M, Kitas GD, van Riel PL. Burden of disease in treated rheumatoid arthritis patients: going beyond the joint. Seminars in arthritis and rheumatism. 2014;43(4):479488.

7. Sokka T, Kautiainen H, Pincus T, et al. Work disability remains a major problem in rheumatoid arthritis in the 2000s: data from 32 countries in the QUEST-RA study. Arthritis research \& therapy. 2010;12(2):R42.

8. Dougados M. Comorbidities in rheumatoid arthritis. Current Opinion in Rheumatology. 2016;28(3):282-288.

9. Wolfe F, Michaud K, Li T, Katz RS. Chronic conditions and health problems in rheumatic diseases: comparisons with rheumatoid arthritis, noninflammatory rheumatic 
disorders, systemic lupus erythematosus, and fibromyalgia. The Journal of rheumatology. 2010;37(2):305-315.

10. Radner H, Yoshida K, Smolen JS, Solomon DH. Multimorbidity and rheumatic conditions-enhancing the concept of comorbidity. Nature reviews Rheumatology. $2014 ; 10(4): 252-256$.

11. Goodson N, Marks J, Lunt M, Symmons D. Cardiovascular admissions and mortality in an inception cohort of patients with rheumatoid arthritis with onset in the 1980s and 1990s. Annals of the Rheumatic Diseases. 2005;64(11):1595-1601.

12. Kojima M, Kojima T, Suzuki S, et al. Depression, inflammation, and pain in patients with rheumatoid arthritis. Arthritis and rheumatism. 2009;61(8):1018-1024.

13. Capuron L, Miller AH. Cytokines and psychopathology: lessons from interferon-alpha. Biological psychiatry. 2004;56(11):819-824.

14. Musselman DL, Lawson DH, Gumnick JF, et al. Paroxetine for the prevention of depression induced by high-dose interferon alfa. The New England journal of medicine. 2001;344(13):961-966.

15. Matcham F, Rayner L, Steer S, Hotopf M. The prevalence of depression in rheumatoid arthritis: a systematic review and meta-analysis. Rheumatology (Oxford, England). 2013;52(12):2136-2148.

16. Jacob L, Rockel T, Kostev K. Depression Risk in Patients with Rheumatoid Arthritis in the United Kingdom. Rheumatology and Therapy. 2017;4(1):195-200.

17. Lin M-C, Guo H-R, Lu M-C, Livneh H, Lai N-S, Tsai T-Y. Increased risk of depression in patients with rheumatoid arthritis: a seven-year population-based cohort study. Clinics. 2015;70(2):91-96. 
18. Bruce TO. Comorbid depression in rheumatoid arthritis: Pathophysiology and clinical implications. Current Psychiatry Reports. 2008;10(3):258-264.

19. Euesden J, Danese A, Lewis CM, Maughan B. A bidirectional relationship between depression and the autoimmune disorders-New perspectives from the National Child Development Study. PLoS One. 2017;12(3):e0173015.

20. Lu M-C, Guo H-R, Lin M-C, Livneh H, Lai N-S, Tsai T-Y. Bidirectional associations between rheumatoid arthritis and depression: a nationwide longitudinal study. Scientific Reports. 2016;6:20647.

21. Euesden J, Danese A, Lewis CM, Maughan B. A bidirectional relationship between depression and the autoimmune disorders - New perspectives from the National Child Development Study. PLoS One. 2017;12(3):e0173015.

22. Ang DC, Choi H, Kroenke K, Wolfe F. Comorbid depression is an independent risk factor for mortality in patients with rheumatoid arthritis. The Journal of rheumatology. 2005;32(6):1013-1019.

23. Scherrer JF, Virgo KS, Zeringue A, et al. Depression increases risk of incident myocardial infarction among Veterans Administration patients with rheumatoid arthritis. General hospital psychiatry. 2009;31(4):353-359.

24. Liu YL, Szklo M, Davidson KW, Bathon JM, Giles JT. Differential Association of Psychosocial Comorbidities With Subclinical Atherosclerosis in Rheumatoid Arthritis. Arthritis care \& research. 2015;67(10):1335-1344.

25. Tektonidou MG, Dasgupta A, Ward MM. Suicidal ideation among adults with arthritis: Prevalence and subgroups at highest risk: Data from the 2007-2008 National Health and Nutrition Examination Survey. Arthritis care \& research. 2011;63(9):1322-1333. 
26. Fuller-Thomson E, Shaked Y. Factors associated with depression and suicidal ideation among individuals with arthritis or rheumatism: findings from a representative community survey. Arthritis and rheumatism. 2009;61(7):944-950.

27. Pinho de Oliveira Ribeiro N, Rafael de Mello Schier A, Ornelas AC, Pinho de Oliveira CM, Nardi AE, Silva AC. Anxiety, depression and suicidal ideation in patients with rheumatoid arthritis in use of methotrexate, hydroxychloroquine, leflunomide and biological drugs. Comprehensive psychiatry. 2013;54(8):1185-1189.

28. Matcham F, Norton S, Scott DL, Steer S, Hotopf M. Symptoms of depression and anxiety predict treatment response and long-term physical health outcomes in rheumatoid arthritis: secondary analysis of a randomized controlled trial. Rheumatology (Oxford, England). 2016;55(2):268-278.

29. Rathbun AM, Harrold LR, Reed GW. A Prospective Evaluation of the Effects of Prevalent Depressive Symptoms on Disease Activity in Rheumatoid Arthritis Patients Treated With Biologic Response Modifiers. Clinical Therapeutics. 2016;38(7):17591772.e1753.

30. Michelsen B, Kristianslund EK, Sexton J, et al. Do depression and anxiety reduce the likelihood of remission in rheumatoid arthritis and psoriatic arthritis? Data from the prospective multicentre NOR-DMARD study. Annals of the Rheumatic Diseases. 2017;76(11):1906-1910.

31. Matcham F, Ali S, Hotopf M, Chalder T. Psychological correlates of fatigue in rheumatoid arthritis: A systematic review. Clinical psychology review. 2015;39(Supplement C):16-29. 
32. Hider SL, Tanveer W, Brownfield A, Mattey DL, Packham JC. Depression in RA patients treated with anti-TNF is common and under-recognized in the rheumatology clinic. Rheumatology. 2009;48(9):1152-1154.

33. Birch JT, Jr., Bhattacharya S. Emerging trends in diagnosis and treatment of rheumatoid arthritis. Primary care. 2010;37(4):779-792, vii.

34. Kojima M, Kojima T, Suzuki S, et al. Depression, inflammation, and pain in patients with rheumatoid arthritis. Arthritis care \& research. 2009;61(8):1018-1024.

35. Uhlig T, Loge JH, Kristiansen IS, Kvien TK. Quantification of reduced health-related quality of life in patients with rheumatoid arthritis compared to the general population. The Journal of rheumatology. 2007;34(6):1241-1247.

36. Gerhold K, Richter A, Schneider M, et al. Health-related quality of life in patients with long-standing rheumatoid arthritis in the era of biologics: data from the German biologics register RABBIT. Rheumatology. 2015;54(10):1858-1866.

37. Katz P. Function, disability, and psychological well-being. In: Pain and Depression. Vol 25. Karger Publishers; 2006:41-62.

38. Katz PP, Morris A, Yelin EH. Prevalence and predictors of disability in valued life activities among individuals with rheumatoid arthritis. Annals of the Rheumatic Diseases. 2006;65(6):763-769.

39. Allaire S, Wolfe F, Niu J, Lavalley MP. Contemporary prevalence and incidence of work disability associated with rheumatoid arthritis in the US. Arthritis care \& research. 2008;59(4):474-480. 
40. Nas K, Sarac AJ, Gur A, et al. Psychological status is associated with health related quality of life in patients with rheumatoid arthritis. Journal of back and musculoskeletal rehabilitation. 2011;24(2):95-100.

41. Cadena J, Vinaccia S, Pérez A, Rico MI, Hinojosa R, Anaya J-M. The impact of disease activity on the quality of life, mental health status, and family dysfunction in colombian patients with rheumatoid arthritis. JCR: Journal Of Clinical Rheumatology. 2003;9(3):142-150.

42. Bazzichi L, Maser J, Piccinni A, et al. Quality of life in rheumatoid arthritis: impact of disability and lifetime depressive spectrum symptomatology. Clinical and experimental rheumatology. 2005;23(6):783-788.

43. Rupp I, Boshuizen HC, Dinant HJ, Jacobi CE, van den Bos GA. Disability and healthrelated quality of life among patients with rheumatoid arthritis: association with radiographic joint damage, disease activity, pain, and depressive symptoms. Scandinavian journal of rheumatology. 2006;35(3):175-181.

44. Kawatkar AA, Jacobsen SJ, Levy GD, Medhekar SS, Venkatasubramaniam KV, Herrinton LJ. Direct medical expenditure associated with rheumatoid arthritis in a nationally representative sample from the medical expenditure panel survey. Arthritis Care Res (Hoboken). 2012;64(11):1649-1656.

45. Gunnarsson C, Chen J, Rizzo JA, Ladapo JA, Naim A, Lofland JH. The Employee Absenteeism Costs of Rheumatoid Arthritis: Evidence From US National Survey Data. $J$ Occup Environ Med. 2015;57(6):635-642. 
46. Filipovic I, Walker D, Forster F, Curry AS. Quantifying the economic burden of productivity loss in rheumatoid arthritis. Rheumatology (Oxford, England). 2011;50(6):1083-1090.

47. Katz PP, Yelin EH. Prevalence and correlates of depressive symptoms among persons with rheumatoid arthritis. The Journal of rheumatology. 1993;20(5):790-796.

48. Mattey DL, Dawes PT, Hassell AB, Brownfield A, Packham JC. Effect of psychological distress on continuation of anti-tumor necrosis factor therapy in patients with rheumatoid arthritis. The Journal of rheumatology. 2010;37(10):2021-2024.

49. Sambamoorthi U, Shah D, Zhao X. Healthcare burden of depression in adults with arthritis. Expert review of pharmacoeconomics \& outcomes research. 2017;17(1):53-65.

50. World Health Organization. Chronic rheumatic conditions. 2017; http://www.who.int/chp/topics/rheumatic/en/. Accessed October 5, 2017.

51. CDC. Current depression among adults---United States, 2006 and 2008. MMWR Morbidity and mortality weekly report. 2010;59(38):1229.

52. Broadhead WE, Blazer DG, George LK, Tse CK. Depression, disability days, and days lost from work in a prospective epidemiologic survey. Jama. 1990;264(19):2524-2528.

53. Whooley MA, Kiefe CI, Chesney MA, Markovitz JH, Matthews K, Hulley SB. Depressive symptoms, unemployment, and loss of income: The CARDIA Study. Arch Intern Med. 2002;162(22):2614-2620.

54. Barrett EM, Scott DG, Wiles NJ, Symmons DP. The impact of rheumatoid arthritis on employment status in the early years of disease: a UK community-based study. Rheumatology (Oxford, England). 2000;39(12):1403-1409. 
55. Curtis JR, Singh JA. Use of biologics in rheumatoid arthritis: current and emerging paradigms of care. Clinical therapeutics. 2011;33(6):679-707.

56. Scott DL. Biologics-based therapy for the treatment of rheumatoid arthritis. Clinical pharmacology and therapeutics. 2012;91(1):30-43.

57. Singh JA, Saag KG, Bridges SL, Jr., et al. 2015 American College of Rheumatology Guideline for the Treatment of Rheumatoid Arthritis. Arthritis \& rheumatology (Hoboken, NJ). 2016;68(1):1-26.

58. Weinblatt ME, Keystone EC, Furst DE, et al. Adalimumab, a fully human anti-tumor necrosis factor alpha monoclonal antibody, for the treatment of rheumatoid arthritis in patients taking concomitant methotrexate: the ARMADA trial. Arthritis and rheumatism. 2003;48(1):35-45.

59. Moreland LW, Schiff MH, Baumgartner SW, et al. Etanercept therapy in rheumatoid arthritis. A randomized, controlled trial. Annals of internal medicine. 1999;130(6):478486.

60. Maini R, St Clair EW, Breedveld F, et al. Infliximab (chimeric anti-tumour necrosis factor alpha monoclonal antibody) versus placebo in rheumatoid arthritis patients receiving concomitant methotrexate: a randomised phase III trial. ATTRACT Study Group. Lancet (London, England). 1999;354(9194):1932-1939.

61. Yazici Y, Shi N, John A. Utilization of biologic agents in rheumatoid arthritis in the United States: analysis of prescribing patterns in 16,752 newly diagnosed patients and patients new to biologic therapy. Bulletin of the NYU hospital for joint diseases. 2008;66(2):77-85. 
62. Crane MM, Juneja M, Allen J, et al. Epidemiology and Treatment of New-Onset and Established Rheumatoid Arthritis in an Insured US Population. Arthritis care \& research. 2015;67(12):1646-1655.

63. Köhler O, Krogh J, Mors O, Benros ME. Inflammation in Depression and the Potential for Anti-Inflammatory Treatment. Current Neuropharmacology. 2016;14(7):732-742.

64. Sotelo JL, Nemeroff CB. Depression as a systemic disease. Personalized Medicine in Psychiatry. 2017;1-2(Supplement C):11-25.

65. Kappelmann N, Lewis G, Dantzer R, Jones PB, Khandaker GM. Antidepressant activity of anti-cytokine treatment: a systematic review and meta-analysis of clinical trials of chronic inflammatory conditions. Molecular psychiatry. 2016.

66. Kohler O, Benros ME, Nordentoft M, et al. Effect of anti-inflammatory treatment on depression, depressive symptoms, and adverse effects: a systematic review and metaanalysis of randomized clinical trials. JAMA psychiatry. 2014;71(12):1381-1391.

67. Kekow J, Moots R, Khandker R, Melin J, Freundlich B, Singh A. Improvements in patient-reported outcomes, symptoms of depression and anxiety, and their association with clinical remission among patients with moderate-to-severe active early rheumatoid arthritis. Rheumatology (Oxford). 2011;50(2):401-409.

68. Abbott R, Whear R, Nikolaou V, et al. Tumour necrosis factor-alpha inhibitor therapy in chronic physical illness: A systematic review and meta-analysis of the effect on depression and anxiety. Journal of psychosomatic research. 2015;79(3):175-184.

69. Andersen RM. Revisiting the Behavioral Model and Access to Medical Care: Does it Matter? Journal of Health and Social Behavior. 1995;36(1):1-10. 
70. Wilkinson RG, Marmot M. Social determinants of health: the solid facts. World Health Organization; 2003.

71. Dickens C, McGowan L, Clark-Carter D, Creed F. Depression in rheumatoid arthritis: a systematic review of the literature with meta-analysis. Psychosomatic medicine. 2002;64(1):52-60.

72. Matcham F, Rayner L, Steer S, Hotopf M. The prevalence of depression in rheumatoid arthritis: a systematic review and meta-analysis. Rheumatology (Oxford, England). 2013;52(12):2136-2148.

73. Ang DC, Choi H, Kroenke K, Wolfe F. Comorbid depression is an independent risk factor for mortality in patients with rheumatoid arthritis. The Journal of rheumatology. 2005;32(6):1013-1019.

74. Joyce AT, Smith P, Khandker R, Melin JM, Singh A. Hidden cost of rheumatoid arthritis (RA): estimating cost of comorbid cardiovascular disease and depression among patients with RA. The Journal of rheumatology. 2009;36(4):743-752.

75. Theis KA, Murphy L, Hootman JM, Helmick CG, Yelin E. Prevalence and Correlates of Arthritis-Attributable Work Limitation in the US Population Among Persons Ages 1864: 2002 National Health Interview Survey Data. Arthritis and rheumatism. 2007;57(3):355-363.

76. Stein MB, Cox BJ, Afifi TO, Belik SL, Sareen J. Does co-morbid depressive illness magnify the impact of chronic physical illness? A population-based perspective. Psychological medicine. 2006;36(5):587-596.

77. Uhm DC, Nam ES, Lee HY, Lee EB, Yoon YI, Chai GJ. [Health-related quality of life in Korean patients with rheumatoid arthritis: association with pain, disease activity, 
disability in activities of daily living and depression]. Journal of Korean Academy of Nursing. 2012;42(3):434-442.

78. Abu Al-Fadl EM, Ismail MA, Thabit M, El-Serogy Y. Assessment of health-related quality of life, anxiety and depression in patients with early rheumatoid arthritis. The Egyptian Rheumatologist. 2014;36(2):51-56.

79. Mikuls T, Saag K, Criswell L, Merlino L, Cerhan JR. Health related quality of life in women with elderly onset rheumatoid arthritis. The Journal of rheumatology. 2003;30(5):952-957.

80. Joshi N, Khanna R, Shah RM. Relationship between depression and physical activity, disability, burden, and health-related quality of life among patients with arthritis. Population health management. 2015;18(2):104-114.

81. Joyce AT, Smith P, Khandker R, Melin JM, Singh A. Hidden Cost of Rheumatoid Arthritis (RA): Estimating Cost of Comorbid Cardiovascular Disease and Depression Among Patients with RA. The Journal of rheumatology. 2009.

82. Sommers JP. An examination of state estimates using multiple years of data from the medical expenditure panel survey, household component. Agency for Healthcare Research and Quality; 2006.

83. Gaskin DJ, Richard P. The economic costs of pain in the United States. The journal of pain : official journal of the American Pain Society. 2012;13(8):715-724.

84. Ware J, Jr., Kosinski M, Keller SD. A 12-Item Short-Form Health Survey: construction of scales and preliminary tests of reliability and validity. Medical care. 1996;34(3):220233. 
85. Crystal S, Johnson RW, Harman J, Sambamoorthi U, Kumar R. Out-of-pocket health care costs among older Americans. The journals of gerontology Series B, Psychological sciences and social sciences. 2000;55(1):S51-62.

86. Paez KA, Zhao L, Hwang W. Rising out-of-pocket spending for chronic conditions: a ten-year trend. Health affairs (Project Hope). 2009;28(1):15-25.

87. Deb A, Sambamoorthi U, Thornton JD, Schreurs B, Innes K. Direct medical expenditures associated with Alzheimer's and related dementias (ADRD) in a nationally representative sample of older adults - an excess cost approach. Aging \& Mental Health. 2017:1-6.

88. Basu A, Rathouz PJ. Estimating marginal and incremental effects on health outcomes using flexible link and variance function models. Biostatistics. 2005;6(1):93-109.

89. AHRQ;. MEPS HC-036: 1996-2007 Pooled Estimation File. 2009; https://meps.ahrq.gov/data_stats/download_data/pufs/h36/h36u07doc.shtml.

90. Coughlan D, Yeh ST, O'Neill C, Frick KD. Evaluating Direct Medical Expenditures Estimation Methods of Adults Using the Medical Expenditure Panel Survey: An Example Focusing on Head and Neck Cancer. Value in Health. 2014;17(1):90-97.

91. Lin EB, Katon W, Von Korff M, et al. Effect of improving depression care on pain and functional outcomes among older adults with arthritis: A randomized controlled trial. JAMA. 2003;290(18):2428-2429.

92. Gaisin IR, Ivanova LV. FRI0239 Decreased prevalence of anxiety and depression in rheumatoid arthritis patients following $\mathrm{b}$ cell-targeted biologic therapy. Annals of the Rheumatic Diseases. 2013;72(Suppl 3):A454-A454.

93. Orbai A-M, Bingham CO. Patient Reported Outcomes in Rheumatoid Arthritis Clinical Trials. Current rheumatology reports. 2015;17(4):501-501. 
94. Ward MM, Guthrie LC, Alba MI. Brief report: rheumatoid arthritis response criteria and patient-reported improvement in arthritis activity: is an American College of Rheumatology twenty percent response meaningful to patients? Arthritis \& rheumatology (Hoboken, NJ). 2014;66(9):2339-2343.

95. Egede LE, Bishu KG, Walker RJ, Dismuke CE. Impact of diagnosed depression on healthcare costs in adults with and without diabetes: United States, 2004-2011. Journal of affective disorders. 2016;195:119-126.

96. Stewart WF, Ricci JA, Chee E, Hahn SR, Morganstein D. Cost of lost productive work time among US workers with depression. Jama. 2003;289(23):3135-3144.

97. Kessler R, White LA, Birnbaum H, et al. Comparative and Interactive Effects of Depression Relative to Other Health Problems on Work Performance in the Workforce of a Large Employer. Journal of Occupational and Environmental Medicine. 2008;50(7):809-816.

98. LaMontagne AD, Martin A, Page KM, et al. Workplace mental health: developing an integrated intervention approach. BMC Psychiatry. 2014;14:131-131.

99. McDaid D, Park AL. Investing in mental health and well-being: findings from the DataPrev project. Health Promotion International. 2011;26(Suppl 1):i108-i139.

100. Margaretten M, Julian L, Katz P, Yelin E. Depression in patients with rheumatoid arthritis: description, causes and mechanisms. International journal of clinical rheumatology. 2011;6(6):617-623.

101. Fiest KM, Hitchon CA, Bernstein CN, et al. Systematic Review and Meta-analysis of Interventions for Depression and Anxiety in Persons With Rheumatoid Arthritis. Journal 
of clinical rheumatology : practical reports on rheumatic \& musculoskeletal diseases. 2017.

102. Curtis JR, Schabert VF, Yeaw J, et al. Use of a validated algorithm to estimate the annual cost of effective biologic treatment for rheumatoid arthritis. Journal of medical economics. 2014;17(8):555-566.

103. Radner H, Yoshida K, Frits M, et al. The impact of multimorbidity status on treatment response in rheumatoid arthritis patients initiating disease-modifying anti-rheumatic drugs. Rheumatology. 2015;54(11):2076-2084.

104. Bair MJ, Robinson RL, Katon W, Kroenke K. Depression and pain comorbidity: A literature review. Archives of Internal Medicine. 2003;163(20):2433-2445.

105. Sotelo JL, Nemeroff CB. Depression as a systemic disease. Personalized Medicine in Psychiatry. 2017;1:11-25.

106. Imran MY, Saira Khan EA, Ahmad NM, Farman Raja S, Saeed MA, Ijaz Haider I. Depression in Rheumatoid Arthritis and its relation to disease activity. Pakistan Journal of Medical Sciences. 2015;31(2):393-397.

107. Mostafa H, Radwan A. The relationship between disease activity and depression in Egyptian patients with rheumatoid arthritis. The Egyptian Rheumatologist. 2013;35(4):193-199.

108. Li X, Gignac MA, Anis AH. Workplace, psychosocial factors, and depressive symptoms among working people with arthritis: a longitudinal study. The Journal of rheumatology. 2006;33(9):1849-1855. 
109. Curtis JR, Baddley JW, Yang S, et al. Derivation and preliminary validation of an administrative claims-based algorithm for the effectiveness of medications for rheumatoid arthritis. Arthritis research \& therapy. 2011;13(5):R155.

110. Curtis JR, Chastek B, Becker L, et al. Cost and effectiveness of biologics for rheumatoid arthritis in a commercially insured population. Journal of managed care \& specialty pharmacy. 2015;21(4):318-329.

111. Chia VM, O'Malley CD, Danese MD, et al. Prevalence and incidence of comorbidities in elderly women with ovarian cancer. Gynecologic oncology. 2013;129(2):346-352.

112. Findley PA, Shen C, Sambamoorthi U. Depression Treatment Patterns among Elderly with Cancer. Depression Research and Treatment. 2012;2012:9.

113. Fairlie RW. An extension of the Blinder-Oaxaca decomposition technique to logit and probit models. Journal of economic and social measurement. 2005;30(4):305-316.

114. Yun M-S. Decomposing differences in the first moment. Economics letters. 2004;82(2):275-280.

115. Sturgeon JA, Finan PH, Zautra AJ. Affective disturbance in rheumatoid arthritis: psychological and disease-related pathways. 2016;12:532.

116. Mcnamara D. Depression interferes with anti-TNF- $\alpha$ therapy. Rheumatology News. $2007 ; 6(6): 1-3$

117. Lisitsyna TA, Veltishchev D, Seravina O, et al. SAT0090 Depression Recovery Improves the Efficacy of Rheumatoid Arthritis Treatment. Annals of the Rheumatic Diseases. 2013;72(Suppl 3):A610-A610. 
118. Krishnadas R, Krishnadas R, Cavanagh J. Sustained remission of rheumatoid arthritis with a specific serotonin reuptake inhibitor antidepressant: a case report and review of the literature. Journal of Medical Case Reports. 2011;5:112-112.

119. Perrot S, Javier RM, Marty M, Le Jeunne C, Laroche F. Is there any evidence to support the use of anti-depressants in painful rheumatological conditions? Systematic review of pharmacological and clinical studies. Rheumatology. 2008;47(8):1117-1123.

120. Coplan JD, Aaronson CJ, Panthangi V, Kim Y. Treating comorbid anxiety and depression: Psychosocial and pharmacological approaches. World Journal of Psychiatry. 2015;5(4):366-378.

121. Hirschfeld RMA. The Comorbidity of Major Depression and Anxiety Disorders: Recognition and Management in Primary Care. Primary Care Companion to The Journal of Clinical Psychiatry. 2001;3(6):244-254.

122. Matcham F, Norton S, Scott DL, Steer S, Hotopf M. Symptoms of depression and anxiety predict treatment response and long-term physical health outcomes in rheumatoid arthritis: secondary analysis of a randomized controlled trial. Rheumatology (Oxford, England). 2016;55(2):268-278.

123. Scherrer JF, Salas J, Copeland LA, et al. Prescription opioid duration, dose, and increased risk of depression in 3 large patient populations. The Annals of Family Medicine. 2016;14(1):54-62.

124. Brown ES. Effects of glucocorticoids on mood, memory, and the hippocampus. Annals of the New York Academy of Sciences. 2009;1179(1):41-55.

125. Myasoedova E, Davis JM, 3rd, Crowson CS, Gabriel SE. Epidemiology of rheumatoid arthritis: rheumatoid arthritis and mortality. Curr Rheumatol Rep. 2010;12(5):379-385. 
126. Cutolo M, Kitas GD, van Riel PLCM. Burden of disease in treated rheumatoid arthritis patients: Going beyond the joint. Seminars in arthritis and rheumatism.43(4):479-488.

127. Margaretten M, Julian L, Katz P, Yelin E. Depression in patients with rheumatoid arthritis: description, causes and mechanisms. International journal of clinical rheumatology. 2011;6(6):617-623.

128. Dessein PH, Joffe BI, Stanwix AE. High sensitivity C-reactive protein as a disease activity marker in rheumatoid arthritis. The Journal of rheumatology. 2004;31(6):10951097.

129. Low CA, Cunningham AL, Kao AH, Krishnaswami S, Kuller LH, Wasko MC. Association between C-reactive protein and depressive symptoms in women with rheumatoid arthritis. Biological psychology. 2009;81(2):131-134.

130. Louati K, Berenbaum F. Fatigue in chronic inflammation - a link to pain pathways. Arthritis research \& therapy. 2015;17:254.

131. Cadena J, Vinaccia S, Perez A, Rico MI, Hinojosa R, Anaya JM. The impact of disease activity on the quality of life, mental health status, and family dysfunction in colombian patients with rheumatoid arthritis. Journal of clinical rheumatology : practical reports on rheumatic \& musculoskeletal diseases. 2003;9(3):142-150.

132. Iyengar RL, Gandhi S, Aneja A, et al. NSAIDs are associated with lower depression scores in patients with osteoarthritis. The American journal of medicine. 2013;126(11):1017.e1011-1018.

133. Machado DA, Guzman RM, Xavier RM, et al. Open-label observation of addition of etanercept versus a conventional disease-modifying antirheumatic drug in subjects with active rheumatoid arthritis despite methotrexate therapy in the Latin American region. 
Journal of clinical rheumatology : practical reports on rheumatic \& musculoskeletal diseases. 2014;20(1):25-33.

134. Bae SC, Gun SC, Mok CC, et al. Improved health outcomes with etanercept versus usual DMARD therapy in an Asian population with established rheumatoid arthritis. BMC musculoskeletal disorders. 2013;14:13.

135. Kekow J, Moots RJ, Emery P, et al. Patient-reported outcomes improve with etanercept plus methotrexate in active early rheumatoid arthritis and the improvement is strongly associated with remission: the COMET trial. Ann Rheum Dis. 2010;69(1):222-225.

136. Curtis JR, Schabert VF, Harrison DJ, et al. Estimating effectiveness and cost of biologics for rheumatoid arthritis: application of a validated algorithm to commercial insurance claims. Clinical therapeutics. 2014;36(7):996-1004.

137. Control CfD, Prevention. Current depression among adults---United States, 2006 and 2008. MMWR Morbidity and mortality weekly report. 2010;59(38):1229.

138. Callhoff J, Albrecht K, Schett G, Zink A, Westhoff G. Depression is a stronger predictor of the risk to consider work disability in early arthritis than disease activity or response to therapy. RMD Open. 2015;1(1):e000020.

139. Cummings P. The relative merits of risk ratios and odds ratios. Archives of Pediatrics \& Adolescent Medicine. 2009;163(5):438-445.

140. SAS Institute Inc NC. SAS 9.4. 2015. Accessed 10/3/2015, 2015.

141. Uguz F, Akman C, Kucuksarac S, Tufekci O. Anti-tumor necrosis factor-alpha therapy is associated with less frequent mood and anxiety disorders in patients with rheumatoid arthritis. Psychiatry and clinical neurosciences. 2009;63(1):50-55. 
142. Hider SL, Tanveer W, Brownfield A, Mattey DL, Packham JC. Depression in RA patients treated with anti-TNF is common and under-recognized in the rheumatology clinic. Rheumatology (Oxford, England). 2009;48(9):1152-1154.

143. Hetland ML, Christensen IJ, Tarp U, et al. Direct comparison of treatment responses, remission rates, and drug adherence in patients with rheumatoid arthritis treated with adalimumab, etanercept, or infliximab: results from eight years of surveillance of clinical practice in the nationwide Danish DANBIO registry. Arthritis and rheumatism. 2010;62(1):22-32.

144. Wu N, Bhurke S, Shah N, Harrison DJ. Application of a validated algorithm to estimate the effectiveness and cost of biologics for rheumatoid arthritis in the US pharmacy benefit manager context. ClinicoEconomics and Outcomes Research: CEOR. 2015;7:257-266.

145. Oladapo A, Barner JC, Lawson KA, et al. Medication effectiveness with the use of tumor necrosis factor inhibitors among Texas Medicaid patients diagnosed with rheumatoid arthritis. Journal of managed care \& specialty pharmacy. 2014;20(7):657-667.

146. Bluett J, Morgan C, Thurston L, et al. Impact of inadequate adherence on response to subcutaneously administered anti-tumour necrosis factor drugs: results from the Biologics in Rheumatoid Arthritis Genetics and Genomics Study Syndicate cohort. Rheumatology (Oxford, England). 2015;54(3):494-499.

147. Ferrari AJ, Charlson FJ, Norman RE, et al. Burden of depressive disorders by country, sex, age, and year: findings from the global burden of disease study 2010. PLoS medicine. 2013;10(11):e1001547. 
148. Moussavi S, Chatterji S, Verdes E, Tandon A, Patel V, Ustun B. Depression, chronic diseases, and decrements in health: results from the World Health Surveys. The Lancet. 2007;370(9590):851-858.

149. Druss BG, Hwang I, Petukhova M, Sampson NA, Wang PS, Kessler RC. Impairment in role functioning in mental and chronic medical disorders in the United States: results from the National Comorbidity Survey Replication. Molecular psychiatry. 2009;14(7):728-737.

150. Pratt LA, Brody DJ. Depression in the United States household population, 2005-2006. NCHS data brief. 2008(7):1-8.

151. Van Spall HG, Toren A, Kiss A, Fowler RA. Eligibility criteria of randomized controlled trials published in high-impact general medical journals: a systematic sampling review. JAMA : the journal of the American Medical Association. 2007;297(11):1233-1240. 


\section{Appendices}

Appendix 7.1. Chapter 2. Description of Study Sample Working-Age (18 to 64 years) Adults with Rheumatoid Arthritis Medical Expenditure Panel Survey (2009, 2011, 2013 and 2015)

\begin{tabular}{|c|c|c|c|}
\hline & $\mathbf{N}$ & Weighted N & Weighted\% \\
\hline All & 2,662 & $5,719,998$ & 100.0 \\
\hline \multicolumn{4}{|l|}{ Gender } \\
\hline Female & 1,826 & $3,661,958$ & 64.0 \\
\hline Male & 836 & $2,058,040$ & 36.0 \\
\hline \multicolumn{4}{|l|}{ Race/Ethnicity } \\
\hline White & 1,061 & $3,596,249$ & 62.9 \\
\hline African American & 778 & 989,822 & 17.3 \\
\hline Latino & 639 & 780,416 & 13.6 \\
\hline Other & 184 & 353,511 & 6.2 \\
\hline \multicolumn{4}{|l|}{ Age in years } \\
\hline 18-39 years & 444 & 956,652 & 16.7 \\
\hline $40-49$ years & 609 & $1,271,670$ & 22.2 \\
\hline $50-59$ years & 1,080 & $2,326,292$ & 40.7 \\
\hline 60-64 years & 529 & $1,165,384$ & 20.4 \\
\hline \multicolumn{4}{|l|}{ Marital Status } \\
\hline Married & 1,287 & $3,098,420$ & 54.2 \\
\hline Widow/Separated/Divorced & 845 & $1,630,180$ & 28.5 \\
\hline Never married & 530 & 991,398 & 17.3 \\
\hline \multicolumn{4}{|l|}{ Education } \\
\hline Less than High School & 665 & $1,007,827$ & 17.6 \\
\hline High School & 939 & $2,043,963$ & 35.7 \\
\hline More than High School & 1,036 & $2,623,400$ & 45.9 \\
\hline \multicolumn{4}{|l|}{ Poverty Status } \\
\hline Poor & 740 & $1,216,767$ & 21.3 \\
\hline Not Poor & 675 & $1,204,168$ & 21.1 \\
\hline Middle Income & 708 & $1,670,010$ & 29.2 \\
\hline High Income & 539 & $1,629,054$ & 28.5 \\
\hline \multicolumn{4}{|l|}{ Insurance Status } \\
\hline Private & 1,302 & $3,411,533$ & 59.6 \\
\hline Public & 932 & $1,580,322$ & 27.6 \\
\hline Uninsured & 428 & 728,144 & 12.7 \\
\hline \multicolumn{4}{|l|}{ Employment } \\
\hline Employed & 1,309 & $3,071,100$ & 53.7 \\
\hline Not Employed & 1,352 & $2,647,283$ & 46.3 \\
\hline \multicolumn{4}{|l|}{ Region } \\
\hline Northeast & 383 & 984,779 & 17.2 \\
\hline Mid-west & 523 & $1,264,228$ & 22.1 \\
\hline South & 1,190 & $2,454,395$ & 42.9 \\
\hline West & 566 & $1,016,597$ & 17.8 \\
\hline
\end{tabular}


Appendix 7.1. Chapter 2. Description of Study Sample

Working-Age (18 to 64 years) Adults with Rheumatoid Arthritis

Medical Expenditure Panel Survey (2009, 2011, 2013 and 2015)

\begin{tabular}{lrrr}
\hline & $\mathbf{N}$ & Weighted N & Weighted\% \\
\hline All & 2,662 & $5,719,998$ & 100.0 \\
Perceived Physical Health & & & \\
$\quad$ Excellent/very good & 607 & $1,488,614$ & 26.0 \\
$\quad$ Good & 871 & $2,001,187$ & 35.0 \\
$\quad$ Fair/poor & 1,184 & $2,230,197$ & 39.0 \\
Perceived Mental Health & & & 26.0 \\
$\quad$ Excellent/very good & 607 & $1,488,614$ & 35.0 \\
$\quad$ Good & 871 & $2,001,187$ & 39.0 \\
$\quad$ Fair/poor & 1184 & $2,230,197$ & 28.1 \\
Multimorbidity & & & 71.9 \\
$\quad$ RA only & 716 & $1,605,886$ & \\
$\quad$ Multimorbidity & 1,946 & $4,114,112$ & 19.8 \\
Anxiety & & & 80.2 \\
$\quad$ Yes & 494 & $1,134,341$ & \\
$\quad$ No & 2,168 & $4,585,657$ & 44.1 \\
Obesity & & & 53.9 \\
$\quad$ Obese & 1,218 & $2,521,340$ & \\
$\quad$ Not Obese & 1,392 & $3,084,702$ & 27.4 \\
Smoking Status & & & 65.2 \\
$\quad$ Current smoker & 698 & $1,567,457$ & 7.4 \\
$\quad$ Other & 1,755 & $3,729,925$ & 58.1 \\
$\quad$ Missing & 209 & 422,616 & \\
Exercise & & $2,364,802$ & \\
$\quad$ Yes & 1,077 & $3,321,567$ & \\
$\quad$ No & 1,568 & & \\
& & & \\
\hline
\end{tabular}

Note: Based on 2,662 adults with Rheumatoid Arthritis, aged between 18 and 64 years, who were alive during the calendar year. Missing data for the following variables (education, obesity, smoking, and exercise) are not presented.

Weighted $\mathrm{N}$ and percentages were derived by dividing the person weights by the number of years pooled. 
Appendix 7.2. Chapter 3. Baseline Characteristics of Working-Age (18 to 62 years)

RA Patients Initiated on TNFi therapy from

QuintilesIMS RWD Adjudicated Claims Database

\begin{tabular}{|c|c|c|}
\hline & $\mathbf{N}$ & $\%$ \\
\hline Total & 4,222 & \\
\hline \multicolumn{3}{|l|}{ Sex } \\
\hline Female & 3,216 & 76.2 \\
\hline Male & 1,006 & 23.8 \\
\hline \multicolumn{3}{|l|}{ Age } \\
\hline $18-34$ years & 352 & 8.3 \\
\hline $35-44$ years & 732 & 17.3 \\
\hline $45-54$ years & 1,534 & 36.3 \\
\hline 55-62 years & 1,604 & 38.0 \\
\hline \multicolumn{3}{|l|}{ Region } \\
\hline East & 712 & 16.9 \\
\hline Midwest & 1,253 & 29.7 \\
\hline South & 1,917 & 45.4 \\
\hline West & 340 & 8.1 \\
\hline \multicolumn{3}{|l|}{ Insurance Plan Type } \\
\hline HMO & 399 & 9.5 \\
\hline PPO & 3,324 & 78.7 \\
\hline Other & 499 & 11.8 \\
\hline \multicolumn{3}{|l|}{ Index Year } \\
\hline 2010 & 519 & 12.3 \\
\hline 2011 & 1,364 & 32.3 \\
\hline 2012 & 1,276 & 30.2 \\
\hline 2013 & 1,063 & 25.2 \\
\hline \multicolumn{3}{|c|}{ Number of Chronic Conditions } \\
\hline 0 & 1,306 & 30.9 \\
\hline 1 to 3 & 2,487 & 59 \\
\hline$>3$ & 429 & 10 \\
\hline \multicolumn{3}{|l|}{ Anxiety } \\
\hline Yes & 255 & 6.0 \\
\hline No & 3,967 & 94.0 \\
\hline \multicolumn{3}{|l|}{ Substance Abuse } \\
\hline Yes & 50 & 1.2 \\
\hline No & 4,172 & 98.8 \\
\hline \multicolumn{3}{|l|}{ ER Visit } \\
\hline Yes & 944 & 22.4 \\
\hline No & 3,278 & 77.6 \\
\hline \multicolumn{3}{|l|}{ IP Visit } \\
\hline Yes & 348 & 8.2 \\
\hline No & 3,874 & 91.8 \\
\hline \multicolumn{3}{|l|}{ NSAIDS } \\
\hline Yes & 2,325 & 55.1 \\
\hline No & 1,897 & 44.9 \\
\hline \multicolumn{3}{|l|}{ Methotrexate } \\
\hline Yes & 3,104 & 73.5 \\
\hline No & 1,118 & 26.5 \\
\hline \multicolumn{3}{|l|}{ Other DMARDS $\dagger$} \\
\hline Yes & 2,079 & 49.2 \\
\hline No & 2,143 & 50.8 \\
\hline \multicolumn{3}{|l|}{ Glucocorticoids } \\
\hline Yes & 3,001 & 71.1 \\
\hline No & 1,221 & 28.9 \\
\hline
\end{tabular}

(Continued) 
Narcotics

$\begin{array}{lll}\text { Yes } & 2,315 & 54.8\end{array}$

No

Note: Based on 4,222 working age adults with RA, initiated on Tumor Necrosis Factor Inhibitors between 2010 and 2013, aged between 18 and 62, with continuous enrollment for 3 years in a commercial insurance plan. $\dagger$ Other DMARDS include Sulfasalazine, Leflunomide, Hydroxychloroquine, Azathioprine, Chloroquine, Cyclophosphamide, Cyclosporine, Minocycline, and Sodium Aurothiomalate 
Supplemental Figure 7.1.1. Chapter 3. Flow Diagram of Study Cohort

\begin{tabular}{|c|}
\hline $\begin{array}{c}\text { Individuals initiating TNFi between } \\
1^{\text {st }} \text { October } 2010 \text { and } 30^{\text {th }} \text { September } 2014 \\
(N=179,530)\end{array}$ \\
\hline$\downarrow$ \\
\hline $\begin{array}{l}\text { Diagnosis of RA (714.0) prior to TNFi initiation } \\
\qquad(\mathrm{N}=47,056)\end{array}$ \\
\hline$\downarrow$ \\
\hline $\begin{array}{l}\text { 1-year pre-index and 1-year post index } \\
\text { enrollment } \\
(\mathrm{N}=9.558)\end{array}$ \\
\hline$\downarrow$ \\
\hline $\begin{array}{l}\text { Age between } 18 \text { and } 63 \text { years at the index } \\
\qquad(\mathrm{N}=8,731)\end{array}$ \\
\hline$\downarrow$ \\
\hline $\begin{array}{l}\text { No other biologic indications, NHL, CLL and } \\
\text { HIV AIDS } \\
(\mathrm{N}=6,950)\end{array}$ \\
\hline
\end{tabular}




\section{Supplemental Figure 7.1.1. Chapter 4. Flow Diagram of Study Cohort}

\begin{tabular}{|c|}
\hline $\begin{array}{l}\text { Individuals initiating TNFi between } \\
1^{\text {st }} \text { October } 2010 \text { and } 30^{\text {th }} \text { September } 2013 \\
(\mathrm{~N}=136,927)\end{array}$ \\
\hline$\downarrow$ \\
\hline $\begin{array}{l}\text { Diagnosis of RA (714.0) prior to TNFi initiation } \\
\qquad(\mathrm{N}=41,896)\end{array}$ \\
\hline$\downarrow$ \\
\hline $\begin{array}{l}\text { 1-year pre-index and 2-year post index } \\
\text { enrollment } \\
(\mathrm{N}=5.763)\end{array}$ \\
\hline$\downarrow$ \\
\hline $\begin{array}{l}\text { Age between } 18 \text { and } 62 \text { years at the index } \\
\qquad(\mathrm{N}=5,308)\end{array}$ \\
\hline$\downarrow$ \\
\hline $\begin{array}{l}\text { No other biologic indications, NHL, CLL and } \\
\text { HIV AIDS } \\
(\mathrm{N}=4,967)\end{array}$ \\
\hline$\downarrow$ \\
\hline $\begin{array}{l}\text { Individuals without a diagnosis of depression in } \\
\text { the 1-year pre-index and } 1 \text {-year post-index } \\
\text { treatment period } \\
(\mathrm{N}=4,222)\end{array}$ \\
\hline
\end{tabular}

